\title{
THE MAXIMUM NUMBER OF FACES OF THE MINKOWSKI SUM OF THREE CONVEX POLYTOPES*
}

\author{
Menelaos I. Karavelas,$^{\dagger}$ Christos Konaxis ${ }^{\ddagger}$ Eleni Tzanaki ${ }^{\S}$
}

\begin{abstract}
We derive tight expressions for the maximum number of $k$-faces, $0 \leq k \leq d-1$, of the Minkowski sum, $P_{1}+P_{2}+P_{3}$, of three $d$-dimensional convex polytopes $P_{1}, P_{2}$ and $P_{3}$ in $\mathbb{R}^{d}$, as a function of the number of vertices of the polytopes, for any $d \geq 2$. Expressing the Minkowski sum as a section of the Cayley polytope $\mathcal{C}$ of its summands, counting the $k$-faces of $P_{1}+P_{2}+P_{3}$ reduces to counting the $(k+2)$-faces of $\mathcal{C}$ that contain vertices from each of the three polytopes. In two dimensions our expressions reduce to known results, while in three dimensions, the tightness of our bounds follows by exploiting known tight bounds for the number of faces of $r d$-polytopes in $\mathbb{R}^{d}$, where $r \geq d$. For $d \geq 4$, the maximum values are attained when $P_{1}, P_{2}$ and $P_{3}$ are $d$-polytopes, whose vertex sets are chosen appropriately from three distinct $d$-dimensional moment-like curves.
\end{abstract}

\section{Introduction}

We study the Minkowski sum of three $d$-dimensional convex polytopes, or simply $d$-polytopes, in $\mathbb{R}^{d}$, and derive tight upper bounds for the number of its $k$-faces, for $0 \leq k \leq d-1$, with respect to the number of vertices of the summands. Given two convex polytopes $P_{1}$ and $P_{2}$, their Minkowski sum $P_{1}+P_{2}$ is the set $\left\{p_{1}+p_{2} \mid p_{1} \in P_{1}, p_{2} \in P_{2}\right\}$. This definition extends to any number of (not necessarily convex) sets of points. The Minkowski sum of convex polytopes is the convex hull of the Minkowski sum of the vertices of its summands; naturally, it is convex.

Minkowski sums are widespread operations in Computational Geometry and find applications in a wide range of areas such as robot motion planning [21], pattern recognition [28], collision detection [22], Computer-Aided Design, and, very recently, Game Theory [25]. They reflect geometrically some algebraic operations, and capture important properties of algebraic objects, such as polynomial systems. This makes them especially useful in Computational Algebra, see e.g., [14, 27, 4].

The geometry of the Minkowski sum can be derived from that of its summands: its normal fan is the common refinement of the normal fans of the summands (see [31] for definitions and details). However, its combinatorial structure is not fully understood, partially due to the fact that most algorithms for computing Minkowski sums have focused

\footnotetext{
${ }^{*}$ A short version of this paper has appeared in [17].

${ }^{\dagger}$ Department of Mathematics \& Applied Mathematics, University of Crete, mkaravel@uoc.gr

${ }^{\ddagger}$ Archimedes Center for Modeling, Analysis $\&$ Computation, University of Crete, ckonaxis@acmac.uoc.gr

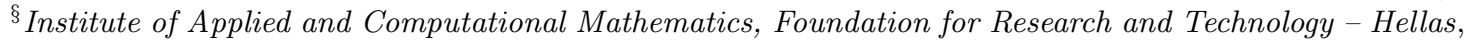
etzanaki@uoc.gr
} 
on low dimensions (see, e.g., [8] for algorithms in three dimensions). The recent development of algorithms that target high dimensions [9], has led to a more extensive study of their properties (see, e.g., [29]).

A natural and fundamental question regarding the combinatorial properties of Minkowski sums concerns their complexity measured as a function of the vertices or the facets of the summands. A complete answer, in terms of the number of vertices of the summands did not exist until very recently [2] (see also Section 7 were we discuss this recent result in more detail). Deriving tight upper bounds with respect to the number of facets of the summands seems harder; results are known only for certain classes of polytopes (see paragraph on previous work below). Knowing the maximum complexity of Minkowski sums allows quantifying the efficiency of algorithms that compute Minkowski sums.

Preliminaries. Let $P$ be a $d$-polytope; its dimension is the dimension of its affine span. The faces of $P$ are $\emptyset, P$, and the intersections of $P$ with its supporting hyperplanes. The $\emptyset$ and $P$ faces are called improper, while the remaining faces are called proper. Each face of $P$ is itself a polytope, and a face of dimension $k$ is called a $k$-face. Faces of $P$ of dimension $0,1, d-2$ and $d-1$ are called vertices, edges, ridges, and facets, respectively.

A $d$-dimensional polytopal complex, or simply $d$-complex, $\mathcal{C}$ is a finite collection of polytopes in $\mathbb{R}^{d}$ such that (i) $\emptyset \in \mathcal{C}$, (ii) if $P \in \mathcal{C}$ then all the faces of $P$ are also in $\mathcal{C}$ and (iii) the intersection $P \cap Q$ for two polytopes $P$ and $Q$ in $\mathcal{C}$ is a face of both. The dimension $\operatorname{dim}(\mathcal{C})$ of $\mathcal{C}$ is the largest dimension of a polytope in $\mathcal{C}$. A polytopal complex is called pure if all its maximal (with respect to inclusion) faces have the same dimension. In this case the maximal faces are called the facets of $\mathcal{C}$. A polytopal complex is simplicial if all its faces are simplices. Finally, a polytopal complex $\mathrm{C}^{\prime}$ is called a subcomplex of a polytopal complex $\mathcal{C}$ if all faces of $\mathcal{C}^{\prime}$ are also faces of $\mathcal{C}$. For a polytopal complex $\mathcal{C}$, the star of $v$ in $\mathcal{C}$, denoted $\operatorname{star}(v, \mathcal{C})$, is the subcomplex of $\mathcal{C}$ consisting of all faces that contain $v$, and their faces. The link of $v$, denoted by $\mathrm{C} / v$, is the $\operatorname{subcomplex}$ of $\operatorname{star}(v, \mathrm{C})$ consisting of all the faces of $\operatorname{star}(v, \mathrm{C})$ that do not contain $v$.

One important class of polytopal complexes arises from polytopes. A $d$-polytope $P$, together with all its faces, form a $d$-complex, denoted by $\mathcal{C}(P)$. The polytope $P$ itself is the only maximal face of $\mathcal{C}(P)$, i.e., the only facet of $\mathcal{C}(P)$, and is called the trivial face of $\mathcal{C}(P)$. Moreover, all proper faces of $P$ form a pure $(d-1)$-complex, called the boundary complex $\mathcal{C}(\partial P)$, or simply $\partial P$, of $P$. The facets of $\partial P$ are just the facets of $P$.

For a $d$-polytope $P$, or its boundary complex $\partial P$, we can define its $f$-vector as $\boldsymbol{f}(P)=\left(f_{-1}, f_{0}, f_{1}, \ldots, f_{d-1}\right)$, where $f_{k}=f_{k}(P)$ denotes the number of $k$-faces of $P$ and $f_{-1}(P):=1$ corresponds to the empty face of $P$. From the $f$-vector of $P$ we define its $h$-vector as the vector $\boldsymbol{h}(P)=\left(h_{0}, h_{1}, \ldots, h_{d}\right)$, where

$$
h_{k}=h_{k}(P)=\sum_{i=0}^{k}(-1)^{k-i}\left(\begin{array}{l}
d-i \\
d-k
\end{array}\right) f_{i-1}(P), \quad 0 \leq k \leq d .
$$

Let $\mathcal{C}$ be a pure simplicial $d$-complex. A shelling $\mathrm{S}(\mathcal{C})$ of $\mathcal{C}$ is a linear ordering $F_{1}, F_{2}, \ldots, F_{s}$ of the facets of $\mathcal{C}$ such that for all $1<j \leq s$ the intersection, $F_{j} \cap\left(\bigcup_{i=1}^{j-1} F_{i}\right)$, 
of the facet $F_{j}$ with the previous facets is non-empty and pure $(d-1)$-dimensional.

Every pure polytopal complex that has a shelling is called shellable. In particular, the boundary complex of a polytope is always shellable (cf. [3]). Consider a pure shellable simplicial polytopal complex $\mathcal{C}$ and let $\mathrm{S}(\mathcal{C})=\left\{F_{1}, \ldots, F_{s}\right\}$ be a shelling order of its facets. The restriction $\mathrm{R}\left(F_{j}\right)$ of a facet $F_{j}$ is the set of all vertices $v \in F_{j}$ such that $F_{j} \backslash\{v\}$ is contained in one of the earlier facets. ${ }^{1}$ The main observation here is that when we construct $\mathcal{C}$ according to the shelling $\mathrm{S}(\mathcal{C})$, the new faces at the $j$ th step of the shelling are exactly the vertex sets $G$ with $\mathrm{R}\left(F_{j}\right) \subseteq G \subseteq F_{j}$ (cf. [31, Section 8.3]). Moreover, notice that $\mathrm{R}\left(F_{1}\right)=\emptyset$ and $\mathrm{R}\left(F_{i}\right) \neq \mathrm{R}\left(F_{j}\right)$ for all $i \neq j$. Using the notion of restrictions we can describe the $h$-vector of a shellable simplicial polytopal complex in geometric terms: $h_{k}(\mathcal{C})$ counts the number of restrictions of size $k$ in a shelling of $\mathcal{C}$, and this number is independent of the particular shelling chosen (cf. [31, Theorem 8.19]).

Previous work. The complexity of Minkowski sums depends on the geometry of their summands. Worst-case tight upper bounds offer the best possible alternative when the geometric characteristics of a specific instance of the problem are not accounted for. Gritzman and Sturmfels [14] were the first to derive tight upper bounds for the number of $k$-faces of $P_{1}+\cdots+P_{r}$, for all $0 \leq k \leq d-1$, and $d, r \geq 2$, namely:

$$
f_{k}\left(P_{1}+\cdots+P_{r}\right) \leq 2\left(\begin{array}{c}
m \\
k
\end{array}\right) \sum_{j=0}^{d-k-1}\left(\begin{array}{c}
m-k-1 \\
j
\end{array}\right),
$$

where $m$ denotes the number of non-parallel edges of $P_{1}, \ldots, P_{r}$. Equality occurs when $P_{i}$ are generic zonotopes, i.e., when each $P_{i}$ is a Minkowski sum of edges, and the generating edges of all polytopes are in general position.

Given two polygons $P_{1}$ and $P_{2}$ in two dimensions with $n_{1}$ and $n_{2}$ vertices (or edges), respectively, their Minkowski sum can have at most $n_{1}+n_{2}$ vertices; clearly, this bound holds also for the number of edges of $P_{1}+P_{2}$, and generalizes in the obvious way for any number of summands (cf. [5]).

In three or more dimensions, Fukuda and Weibel [10] have shown what they call the trivial upper bound: any $k$-face $F$ of the Minkowski sum $\sum_{i=1}^{r} P_{i}$ is a Minkowski sum $F=\sum_{i=1}^{r} F_{i}$ of $k_{i}$-faces $F_{i}$ of $P_{i}$ such that $k=\sum_{i=1}^{r} k_{i}$. Since $P_{i}$ has at most $\left(\begin{array}{c}n_{i} \\ k_{i}+1\end{array}\right) k_{i}$-faces, where $n_{i}$ is the number of vertices of $P_{i}, 1 \leq i \leq r$, the sum

$$
\Phi_{k+r}\left(n_{1}, n_{2}, \ldots, n_{r}\right)=\sum_{\substack{0 \leqslant k_{i} \leqslant d \\
k_{1}+\cdots+k_{r}=k}} \prod_{i=1}^{r}\left(\begin{array}{c}
n_{i} \\
k_{i}+1
\end{array}\right)=\sum_{\substack{1 \leqslant s_{i} \leqslant n_{i} \\
s_{1}+\ldots+s_{r}=k+r}} \prod_{i=1}^{r}\left(\begin{array}{c}
n_{i} \\
s_{i}
\end{array}\right)
$$

bounds the number of $k$-faces of $P_{1}+\cdots+P_{r}$. Hence given $r d$-polytopes $P_{1}, P_{2}, \ldots, P_{r}$ in $\mathbb{R}^{d}$, where $d \geq 3$ and $r \geq 2$, we have, for all $k \geq 0$ :

$$
f_{k}\left(P_{1}+P_{2}+\cdots+P_{r}\right) \leq \Phi_{k+r}\left(n_{1}, n_{2}, \ldots, n_{r}\right) .
$$

\footnotetext{
${ }^{1}$ For simplicial faces, we identify the face with its defining vertex set.
} 
In the same paper, Fukuda and Weibel have shown that the trivial upper bound is tight for: (i) $d \geq 4,2 \leq r \leq\left\lfloor\frac{d}{2}\right\rfloor$ and for all $0 \leq k \leq\left\lfloor\frac{d}{2}\right\rfloor-r$, and (ii) for the number of vertices, $f_{0}\left(P_{1}+P_{2}+\cdots+P_{r}\right)$, of $P_{1}+P_{2}+\cdots+P_{r}$, when $d \geq 3$ and $2 \leq r \leq d-1$. For $r \geq d$, Sanyal [26] has shown that the trivial upper bound for $f_{0}\left(P_{1}+P_{2}+\cdots+P_{r}\right)$ cannot be attained, since in this case:

$$
f_{0}\left(P_{1}+P_{2}+\cdots+P_{r}\right) \leq\left(1-\frac{1}{(d+1)^{d}}\right) \prod_{i=1}^{r} n_{i}<\prod_{i=1}^{r} n_{i}
$$

Karavelas and Tzanaki [18] recently extended the range of of $d, r$ and $k$ for which the trivial upper bound (1) is attained. More precisely, they showed that for any $d \geq 3,2 \leq r \leq d-1$ and for all $0 \leq k \leq\left\lfloor\frac{d+r-1}{2}\right\rfloor-r$, there exist $r$ neighborly $d$-polytopes $P_{1}, P_{2}, \ldots, P_{r}$ in $\mathbb{R}^{d}$, for which the number of $k$-faces of their Minkowski sum attains the trivial upper bound. Recall that a $d$-polytope $P$ is neighborly if any subset of $\left\lfloor\frac{d}{2}\right\rfloor$ or less vertices is the vertex set of a face of $P$. Tight bounds for $f_{0}\left(P_{1}+P_{2}+\cdots+P_{r}\right)$, where $r \geq d$, have very recently been shown by Weibel [30], namely:

$$
f_{0}\left(P_{1}+P_{2}+\cdots+P_{r}\right) \leq \alpha+\sum_{j=1}^{d-1}(-1)^{d-1-j}\left(\begin{array}{c}
r-1-j \\
d-1-j
\end{array}\right) \sum_{S \in X_{j}^{r}}\left(\prod_{i \in S} f_{0}\left(P_{i}\right)-\alpha\right)
$$

where $X_{j}^{r}$ is the family of subsets of $\{1,2, \ldots, r\}$ of cardinality $j$, and $\alpha=2\left(d-2\left\lfloor\frac{d}{2}\right\rfloor\right)$.

Tight bounds for all face numbers, i.e., for all $0 \leq k \leq d-1$, expressed as a function of the number of vertices or facets of the summands, were, until very recently, only known for two $d$-polytopes when $d \geq 3$. Fukuda and Weibel [10] have shown that, given two 3 polytopes $P_{1}$ and $P_{2}$ in $\mathbb{R}^{3}$, the number of $k$-faces of $P_{1}+P_{2}, 0 \leq k \leq 2$, is bounded from above as follows:

$$
\begin{aligned}
& f_{0}\left(P_{1}+P_{2}\right) \leq n_{1} n_{2}, \\
& f_{1}\left(P_{1}+P_{2}\right) \leq 2 n_{1} n_{2}+n_{1}+n_{2}-8, \\
& f_{2}\left(P_{1}+P_{2}\right) \leq n_{1} n_{2}+n_{1}+n_{2}-6,
\end{aligned}
$$

where $n_{i}$ is the number of vertices of $P_{i}, i=1,2$. These bounds are tight. Weibel [29] has derived analogous tight expressions in terms of the number of facets $m_{i}$ of $P_{i}, i=1,2$ :

$$
\begin{aligned}
& f_{0}\left(P_{1}+P_{2}\right) \leq 4 m_{1} m_{2}-8 m_{1}-8 m_{2}+16, \\
& f_{1}\left(P_{1}+P_{2}\right) \leq 8 m_{1} m_{2}-17 m_{1}-17 m_{2}+40, \\
& f_{2}\left(P_{1}+P_{2}\right) \leq 4 m_{1} m_{2}-9 m_{1}-9 m_{2}+26 .
\end{aligned}
$$

Weibel's bound for $f_{2}\left(P_{1}+P_{2}\right)$ (cf. rel. (3)) has been generalized to the number of facets of the Minkowski sum of any number of 3-polytopes by Fogel, Halperin and Weibel [7]; they have shown that, for $r \geq 2$, the following tight bound holds:

$$
f_{2}\left(P_{1}+P_{2}+\cdots+P_{r}\right) \leq \sum_{1 \leq i<j \leq r}\left(2 m_{i}-5\right)\left(2 m_{j}-5\right)+\sum_{i=1}^{r} m_{i}+\left(\begin{array}{l}
r \\
2
\end{array}\right)
$$


where $m_{i}=f_{2}\left(P_{i}\right), 1 \leq i \leq r$. Finally, Karavelas and Tzanaki [19] have shown that for any two $d$-polytopes $P_{1}$ and $P_{2}$ in $\mathbb{R}^{d}$, where $d \geq 4$, and for all $1 \leq k \leq d$, we have:

$$
f_{k-1}\left(P_{1}+P_{2}\right) \leq f_{k}\left(C_{d+1}\left(n_{1}+n_{2}\right)\right)-\sum_{i=0}^{\left\lfloor\frac{d+1}{2}\right\rfloor}\left(\begin{array}{c}
d+1-i \\
k+1-i
\end{array}\right)\left(\left(\begin{array}{c}
n_{1}-d-2+i \\
i
\end{array}\right)+\left(\begin{array}{c}
n_{2}-d-2+i \\
i
\end{array}\right)\right),
$$

where $n_{i}=f_{0}\left(P_{i}\right), i=1,2$, and $C_{d}(n)$ stands for the cyclic $d$-polytope with $n$ vertices. The bounds in (4) have been shown to be tight, and match the corresponding, previously known, bounds for 2- and 3-polytopes (cf. rel. (2)).

Overview. In this work we continue the line of research in [19], extending the methods to deal with the case of three $d$-polytopes in $\mathbb{R}^{d}$. Allowing just one more summand significantly increases the problem's intricacy. In particular, deriving Lemmas 6, 7 and 11, which are essential in proving our upper bounds, requires techniques much more involved than those used in the case of two polytopes. This is also the case when establishing the tightness of the upper bounds in Section 6: in our constructions an additional difficulty had to be overcome, since we require that not only the face numbers of the sum of the three polytopes are maximal, but also those of the three pairwise sums of the three polytopes. Even more importantly, the case of three $d$-polytopes provides a valuable insight towards our ultimate goal, the general case of $r d$-polytopes in $\mathbb{R}^{d}$, for any $d, r \geq 2$. Using the tools and methodology applied in this paper, some of the results obtained here can be generalized to the case $d, r \geq 2$ (see Section 7), while others still remain elusive.

We state our main result:

Theorem 1. Let $P_{1}, P_{2}$ and $P_{3}$ be three d-polytopes in $\mathbb{R}^{d}, d \geq 2$, with $n_{i} \geq d+1$ vertices, $1 \leq i \leq 3$. Then, for all $1 \leq k \leq d$, we have:

$$
\begin{aligned}
f_{k-1}\left(P_{1}+P_{2}+P_{3}\right) \leq & f_{k+1}\left(C_{d+2}\left(n_{[3]}\right)\right)-\sum_{i=0}^{\left\lfloor\frac{d+2}{2}\right\rfloor}\left(\begin{array}{l}
d+2-i \\
k+2-i
\end{array}\right) \sum_{\emptyset \subset S \subset[3]}(-1)^{|S|}\left(\begin{array}{c}
n_{S}-d-3+i \\
i
\end{array}\right) \\
& -\delta\left(\begin{array}{c}
\left\lfloor\frac{d}{2}\right\rfloor+1 \\
k-\left\lfloor\frac{d}{2}\right\rfloor
\end{array}\right) \sum_{i=1}^{3}\left(\begin{array}{c}
n_{i}-\left\lfloor\frac{d}{2}\right\rfloor-2 \\
\left\lfloor\frac{d}{2}\right\rfloor+1
\end{array}\right),
\end{aligned}
$$

where $[3]=\{1,2,3\}, \delta=d-2\left\lfloor\frac{d}{2}\right\rfloor, n_{S}=\sum_{i \in S} n_{i}$, and $C_{d+2}\left(n_{[3]}\right)$ is the cyclic $(d+2)$ polytope with $n_{[3]}$ vertices. Moreover, for any $d \geq 2$, there exist three $d$-polytopes in $\mathbb{R}^{d}$ for which the bounds above are attained for all $1 \leq k \leq d$.

To establish the upper bounds (cf. Section 5) we first lift the three $d$-polytopes in $\mathbb{R}^{d+2}$ using an affine basis of $\mathbb{R}^{2}$, and form the convex hull $\mathcal{C}$ of the embedded polytopes in $\mathbb{R}^{d+2}$. The polytope $\mathcal{C}$ is known as the Cayley polytope of the $P_{i}$ 's. We consider the set $\mathcal{F}_{[3]}$, consisting of the $k$-faces of $\mathcal{C}, 2 \leq k \leq d+1$, that contain at least one vertex from each $P_{i}$. We then exploit the bijection between the $k$-faces of $\mathcal{F}_{[3]}$ and the $(k-2)$-faces of $P_{1}+P_{2}+P_{3}$ (cf. Section 2) to reduce the derivation of upper bounds for $f_{k-2}\left(P_{1}+P_{2}+P_{3}\right)$ and derive upper bounds for $f_{k}\left(\mathcal{F}_{[3]}\right), 2 \leq k \leq d+1$. 
The rest of our upper bound proof follows the main steps of McMullen's proof of the Upper bound Theorem for polytopes [24]. In Section 3 we add auxiliary vertices to appropriate faces of the Cayley polytope $\mathcal{C}$, resulting in a simplicial polytope $\mathcal{Q}$ whose face set contains $\mathcal{F}_{[3]}$. We then consider the $f$-vector $\boldsymbol{f}(\partial \mathcal{Q})$ and the $h$-vector $\boldsymbol{h}(\partial \mathcal{Q})$ of $\partial \mathcal{Q}$ and derive expressions for their entries via the corresponding vectors for $\mathcal{F}_{[3]}$. Using these expressions, we continue by deriving Dehn-Sommerville-like equations for $\mathcal{F}_{[3]}$. As an intermediate step we define the subcomplex $\mathcal{K}_{[3]}$ of $\mathcal{C}$, as the closure under subface inclusion, of $\mathcal{F}_{[3]}$, and derive expressions for its $f$ - and $h$-vectors (cf. relations (5) and (13) with $R=[3]$ ). This allows us to write the Dehn-Sommerville-like equations for $\mathcal{F}_{[3]}$ in the very concise form:

$$
h_{d+2-k}\left(\mathcal{F}_{[3]}\right)=h_{k}\left(\mathcal{K}_{[3]}\right), \quad 0 \leq k \leq d+2 .
$$

In Section 4, using a well known relation by McMullen (cf. rel. (18)), along with the expressions that relate the $h$-vector of $\partial \mathcal{Q}$ with those of $\mathcal{F}_{[3]}$ and $\mathcal{K}_{[3]}$, we establish a recurrence relation for the elements of $\boldsymbol{h}\left(\mathcal{F}_{[3]}\right)$ (see Lemma 8). This recurrence relation for the elements of $\boldsymbol{h}\left(\mathcal{F}_{[3]}\right)$ is then used in Section 5 to prove upper bounds on the elements of $\boldsymbol{h}\left(\mathcal{F}_{[3]}\right)$ and $\boldsymbol{h}\left(\mathcal{K}_{[3]}\right)$. These upper bounds combined with the Dehn-Sommerville-like equations for $\mathcal{F}_{[3]}$, yield refined upper bounds for the values $h_{k}\left(\mathcal{F}_{[3]}\right)$ when $k>\left\lfloor\frac{d+2}{2}\right\rfloor$. We end by establishing our upper bounds on the number of $k$-faces, $0 \leq k \leq d-1$, of $P_{1}+P_{2}+P_{3}$ by computing $\boldsymbol{f}\left(\mathcal{F}_{[3]}\right)$ from $\boldsymbol{h}\left(\mathcal{F}_{[3]}\right)$. At the same time we establish conditions on a subset of the elements of the vectors $\boldsymbol{f}\left(\mathcal{F}_{R}\right), \emptyset \subset R \subseteq[3],{ }^{2}$ that are sufficient and necessary in order for the upper bounds in the number of $k$-faces of $P_{1}+P_{2}+P_{3}$ to be tight for all $k\left(\mathcal{F}_{R}\right.$ stands for the set of faces of $\mathcal{C}$ that have at least one vertex from each $P_{i}$ for all $i \in R$, but no vertex from any $P_{j}$ with $j \notin R$ ).

In Section 6 we describe the constructions that establish the tightness of our upper bounds. For $d=2$ and $d=3$ we rely on previous results. For $d \geq 4$ we define three convex $d$-polytopes, whose vertices lie on three distinct moment-like $d$-curves, and show that the sets $\mathcal{F}_{R}, \emptyset \subset R \subseteq[3]$, associated with them satisfy the sufficient and necessary conditions mentioned above. We conclude with Section 7, where we discuss the case of four or more summands and directions for future work.

\section{The Cayley trick}

Recall that [3] stands for the set $\{1,2,3\}$, and denote by $X_{j}:=\{R \subseteq[3]|| R \mid=j\}$, the set of all subsets of [3] of cardinality $j$, for $1 \leq j \leq 3$. Consider three $d$-polytopes $P_{1}$, $P_{2}$ and $P_{3}$ in $\mathbb{R}^{d}$, and choose the basis $\boldsymbol{e}_{2,1}=(0,0), \boldsymbol{e}_{2,2}=(1,0), \boldsymbol{e}_{2,3}=(0,1)$, as the preferred affine basis of $\mathbb{R}^{2}$. The Cayley embedding of the $P_{i}$ 's is defined via the maps $\mu_{i}(\boldsymbol{x})=\left(\boldsymbol{e}_{2, i}, \boldsymbol{x}\right)$, and we denote by $\mathcal{C}$ the $(d+2)$-polytope we get by taking the convex hull of the sets $\mathcal{V}_{i}=\left\{\mu_{i}(\boldsymbol{v}) \mid v \in V_{i}\right\}$, where $V_{i}$ is the vertex set of $P_{i}$. This is known as the Cayley polytope of the $P_{i}$ 's (see Fig. 1(a)). Similarly, by taking appropriate affine bases, we define the Cayley polytope $\mathcal{C}_{R}$ of all polytopes $P_{i}, i \in R$, where $R \in X_{j}, j=1,2$. These are the Cayley polytopes of all pairs of $P_{i}$ 's (see Fig. 1(b)) and, trivially, the $P_{i}$ 's themselves. Clearly, $\mathcal{C}_{R} \equiv P_{R}$, for $R \in \mathcal{X}_{1}$, and $\mathcal{C} \equiv \mathcal{C}_{[3]}$.

\footnotetext{
${ }^{2}$ The symbol " $\subset$ " refers to the proper subset relationship, also denoted as " $\subsetneq$ " in the literature.
} 
For any $\emptyset \subset R \subseteq[3]$, let $\mathcal{V}_{R}$ denote the union of the sets $\mathcal{V}_{i}, i \in R$. Below we identify $\mathcal{C}_{R} \subset \mathbb{R}^{d+|R|-1}$, for all $R \in X_{j}, j=1,2$, with the affinely isomorphic and combinatorially equivalent polytope $\operatorname{conv}\left(\mathcal{V}_{R}\right) \subset \mathcal{C} \subset \mathbb{R}^{d+2}$. This allows us to study properties of these subsets of $\mathcal{C}$ by examining the corresponding Cayley polytopes that lie in lower dimensional spaces.

We denote by $\mathcal{F}_{R}, \emptyset \subset R \subseteq[3]$, the set of proper faces of $\mathcal{C}_{R}$, with the property that $F \in \mathcal{F}_{R}$ if $F \cap \mathcal{V}_{i} \neq \emptyset$, for all $i \in R$. In other words, $\mathcal{F}_{R}$ consists of all the proper faces of $\mathcal{C}_{R}$ that have at least one vertex from each $\mathcal{V}_{i}$, for all $i \in R$, (see also Figs. $1(\mathrm{c})$ and $1(\mathrm{~d})$ ). Clearly, if $|R| \geq 2$, then $f_{0}\left(\mathcal{F}_{R}\right)=0$. Moreover, if $R \in X_{1}$ then $\mathcal{F}_{R} \equiv \partial P_{R}$. The dimension of $\mathcal{F}_{R}$ is the maximum dimension of the faces in $\mathcal{F}_{R}$, i.e., $\operatorname{dim}\left(\mathcal{F}_{R}\right)=\max _{F \in \mathcal{F}_{R}} \operatorname{dim}(F)=$ $d+|R|-2$.

Let $\bar{W}$ be the $d$-flat of $\mathbb{R}^{d+2}$ :

$$
\bar{W}=\left\{\frac{1}{3} \boldsymbol{e}_{2,1}+\frac{1}{3} \boldsymbol{e}_{2,2}+\frac{1}{3} \boldsymbol{e}_{2,3}\right\} \times \mathbb{R}^{d},
$$

and consider the weighted Minkowski sum $\frac{1}{3} P_{1}+\frac{1}{3} P_{2}+\frac{1}{3} P_{3}$. Note that this is nothing more than $P_{1}+P_{2}+P_{3}$, scaled down by $\frac{1}{3}$, hence these two sums are combinatorially equivalent. The Cayley trick [16] says that the intersection of $\bar{W}$ with $\mathcal{C}$ is combinatorially equivalent (isomorphic) to the weighted Minkowski sum $\frac{1}{3} P_{1}+\frac{1}{3} P_{2}+\frac{1}{3} P_{3}$, hence, also to the unweighted Minkowski sum $P_{1}+P_{2}+P_{3}$ (refer to Fig. 1(a) again). Moreover, every face of $P_{1}+P_{2}+P_{3}$ is the intersection of a face of $\mathcal{F}_{[3]}$ with $\bar{W}$. This implies that:

$$
f_{k-1}\left(P_{1}+P_{2}+P_{3}\right)=f_{k+1}\left(\mathcal{F}_{[3]}\right), \quad 1 \leq k \leq d .
$$

In the rest of the paper we assume that $\mathcal{C}$ is "as simplicial as possible", i.e., all faces of $\mathcal{C}$ are simplicial except for the trivial faces of $\mathcal{C}_{R}$, for all $\emptyset \subset R \subseteq$ [3]. Otherwise, we can employ the so called bottom-vertex triangulation [23, Section 6.5, pp. 160-161], where we triangulate every face of $\mathcal{C}$ except the trivial faces of $\mathcal{C}_{R}$ (i.e., $\mathcal{C}_{R}$ themselves and not their proper faces) for all $\emptyset \subset R \subseteq[3]$. The resulting complex is polytopal (cf. [6]) and all of its faces are simplicial, except for the seven trivial faces above. Moreover, it has the same number of vertices as $\mathcal{C}$, while the number of its $k$-faces is never less than the number of $k$-faces of $\mathcal{C}$.

Under the "as simplicial as possible" assumption above, the faces in $\mathcal{F}_{R}, \emptyset \subset R \subseteq[3]$, are simplicial. We denote by $\mathcal{K}_{R}$ the closure, under subface inclusion, of $\mathcal{F}_{R}$, i.e., $\mathcal{K}_{R}$ contains all the faces in $\mathcal{F}_{R}$ and all the faces that are subfaces of faces in $\mathcal{F}_{R}$. It is easy to see that $\mathcal{K}_{R}$ does not contain any of the trivial faces of $\mathcal{C}_{S}, S \subseteq R$, and, thus, $\mathcal{K}_{R}$ is a pure simplicial $(d+|R|-2)$-complex, whose facets are precisely the facets in $\mathcal{F}_{R}$. It is also clear that $\mathcal{F}_{R} \equiv \mathcal{K}_{R} \equiv \partial P_{R}$, for $R \in \mathcal{X}_{1}$. Moreover, $\mathcal{K}_{[3]}$ is the boundary complex $\partial \mathcal{C}$ of the Cayley polytope $\mathcal{C}$, except for its three facets (i.e., $(d+1)$-faces) $\mathcal{C}_{R}, R \in X_{2}$, and its three ridges (i.e., $d$-faces) $P_{i}, 1 \leq i \leq 3$.

Consider a $k$-face $F$ of $\mathcal{K}_{R}, \emptyset \subset R \subseteq[3]$. By the definition of $\mathcal{K}_{R}, F$ is either a $k$-face of $\mathcal{F}_{R}$, or a $k$-face of $\mathcal{F}_{S}$ for some nonempty subset $S$ of $R$. Hence:

$$
f_{k}\left(\mathcal{K}_{R}\right)=\sum_{\emptyset \subset S \subseteq R} f_{k}\left(\mathcal{F}_{S}\right), \quad-1 \leq k \leq d+|R|-2
$$




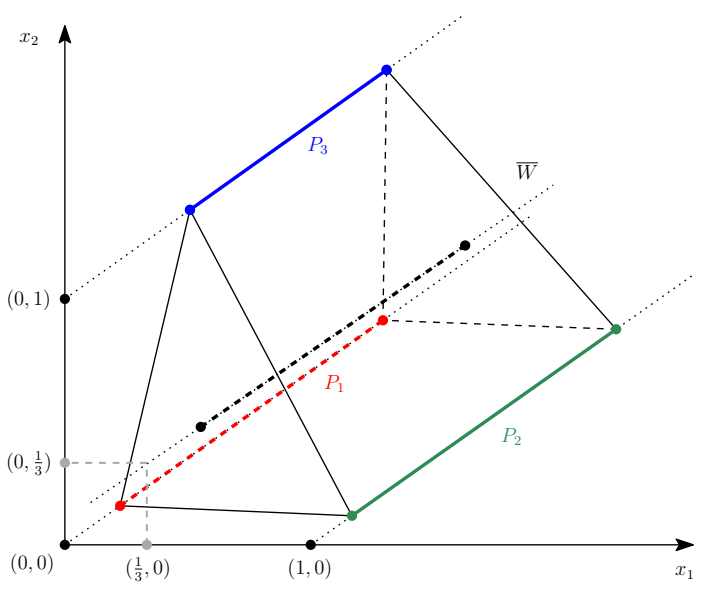

(a)

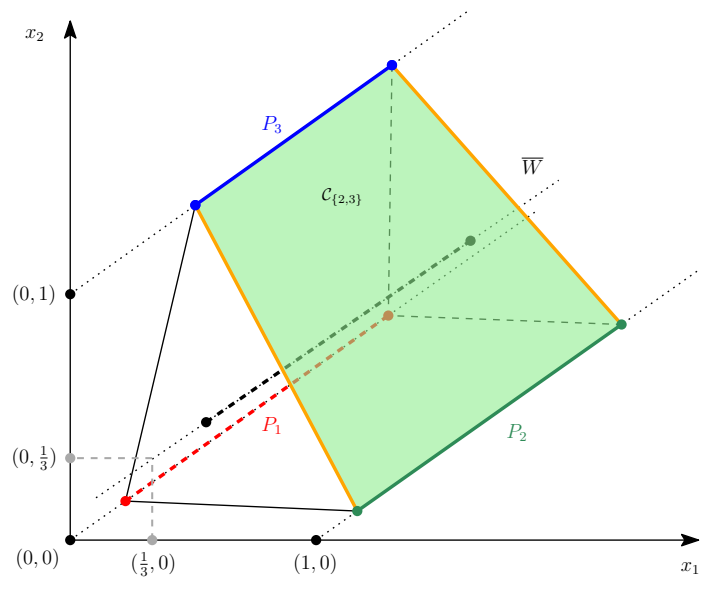

(c)

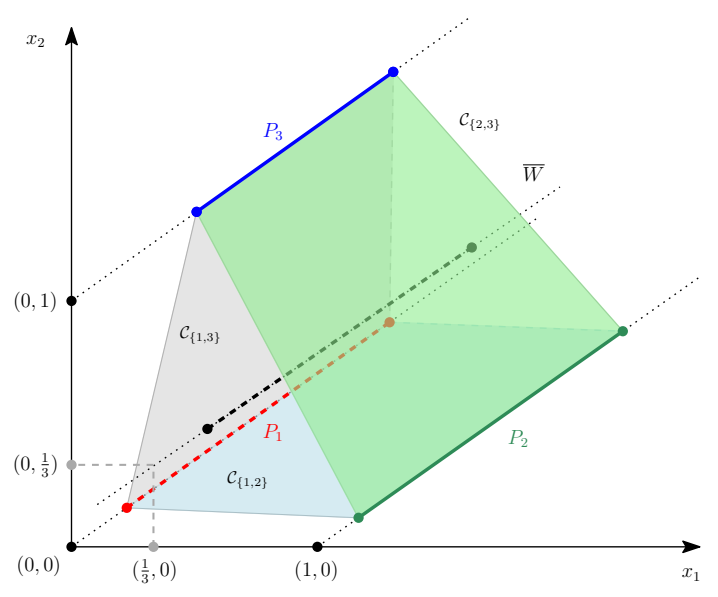

(b)

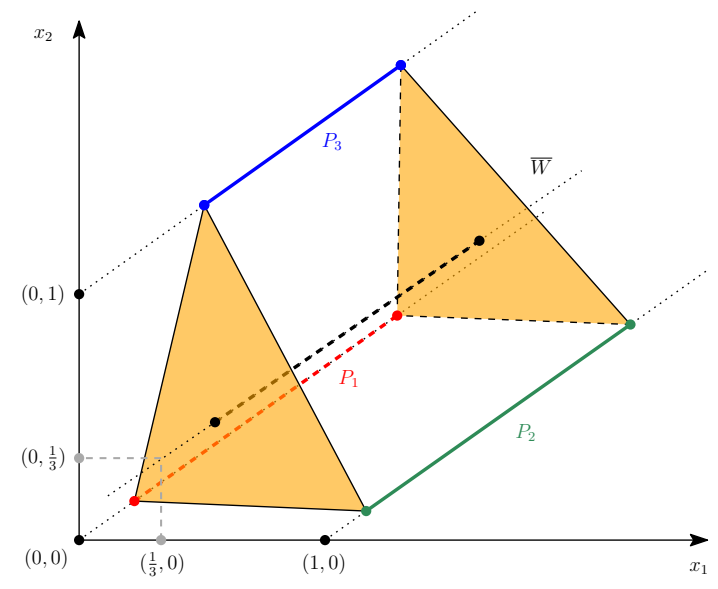

(d)

Figure 1: (a) Schematic of the Cayley trick for three polytopes. The three polytopes $P_{1}$, $P_{2}$ and $P_{3}$ are shown in red, green and blue, respectively. The polytope $\frac{1}{3} P_{1}+\frac{1}{3} P_{2}+\frac{1}{3} P_{3}$ is shown in black; (b) The Cayley polytopes $\mathcal{C}_{R}, R \in X_{2}$ as subsets of $\mathcal{C}: \mathcal{C}_{\{1,2\}}$ (light blue), $\mathcal{C}_{\{1,3\}}$ (gray), $\mathcal{C}_{\{2,3\}}$ (green); (c) The Cayley polytope $\mathcal{C}_{\{2,3\}}$ (green) and the set $\mathcal{F}_{\{2,3\}}$ (orange); (d) The set of faces $\mathcal{F}_{[3]}$ (orange) of the Cayley polytope $\mathcal{C}$.

where, in order for the above equation to hold for $k=-1$, we set $f_{-1}\left(\mathcal{F}_{R}\right)=(-1)^{|R|-1}$. By inclusion-exclusion, we can easily see that

$$
f_{k}\left(\mathcal{F}_{R}\right)=\sum_{\emptyset \subset S \subseteq R}(-1)^{|R|-|S|} f_{k}\left(\mathcal{K}_{S}\right), \quad-1 \leq k \leq d+|R|-2 .
$$

In what follows we use the convention that $f_{k}\left(\mathcal{F}_{R}\right)=0$, for any $k<-1$ or $k>$ $d+|R|-2$. 


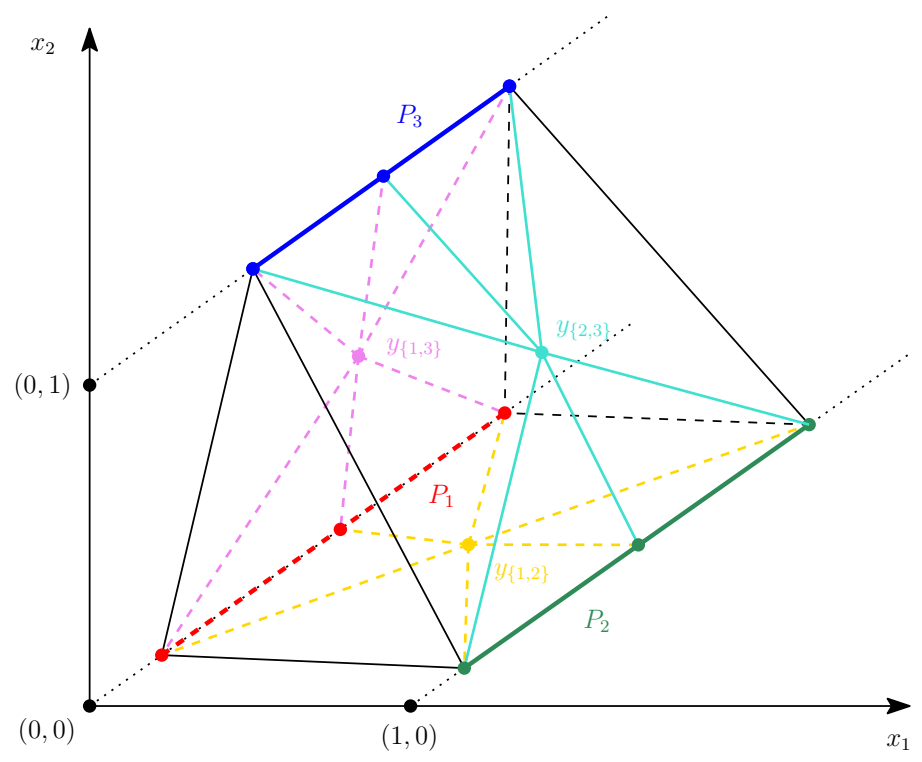

Figure 2: The $(d+2)$-polytope $\mathcal{Q}$.

\section{$3 f$-vectors, $h$-vectors and Dehn-Sommerville-like equations}

The Cayley polytope $\mathcal{C}$ need not be simplicial. In order to construct a simplicial polytope $\mathcal{Q}$ from $\mathcal{C}$, we define auxiliary vertices in $\mathbb{R}^{d+2}$ not contained in $\mathcal{V}_{i}, i=1,2,3$. The Dehn-Sommerville equations of $\mathcal{Q}$ will be used, later on in this section, to derive DehnSommerville-like equations for $\mathcal{F}_{[3]}$ and $\mathcal{K}_{[3]}$.

For every $\emptyset \subset R \subset[3]$ we add a vertex $y_{R}$ in the relative interior of $\mathcal{C}_{R}$ and, following [6], we consider the complex arising by taking successive stellar subdivisions of $\partial \mathcal{C}$ as follows:

(i) we form the complex arising from $\partial \mathcal{C}$ by taking the stellar subdivisions $\operatorname{st}\left(y_{\{i\}}, \mathcal{C}_{\{i\}}\right)$ for all $1 \leq i \leq 3$, then

(ii) we form the complex arising from the one constructed in the previous step by taking the stellar subdivisions $\operatorname{st}\left(y_{R}, \mathcal{C}_{R}^{\prime}\right)$ for every $R \in X_{2}$. $\mathcal{C}_{R}^{\prime}$ is the complex obtained by taking, for every $S \subset R$, the stellar subdivision of $y_{S}$ over the boundary complex of $\mathcal{C}_{S}$

This complex is polytopal and isomorphic to the boundary complex of a $(d+2)$-polytope, which we denote as $\mathcal{Q}$ (see also Fig. 2). The boundary complex $\partial \mathcal{Q}$ is a simplicial $(d+1)$ sphere. The simpliciality of $\partial \mathcal{Q}$ allows us to utilize its Denh-Sommerville equations in order to prove Dehn-Sommerville-like equations for $\mathcal{F}_{[3]}$ in the upcoming Lemma 4 . We denote by $\mathcal{V}:=\mathcal{V}_{1} \cup \mathcal{V}_{2} \cup \mathcal{V}_{3} \cup\left\{y_{R} \mid \emptyset \subset R \subset[3]\right\}$ the vertex set of $\mathcal{Q}$.

Let us count the $k$-faces of $\partial \mathcal{Q}$. Suppose that $F$ is a $k$-face of $\partial \mathcal{Q}$. We distinguish between the following cases depending on the number of auxiliary vertices, $y_{R}$, that $F$ 
contains:

(i) $F$ does not contain any additional auxiliary vertices. Then, it can be a $k$-face of any $\mathcal{F}_{R}, R \in X_{1}$, or it can be a $k$-face of any of the $\mathcal{F}_{R}, R \in X_{2}$, or it can be a $k$-face of $\mathcal{F}_{[3]}$. This gives a total of $f_{k}\left(\mathcal{F}_{[3]}\right)+\sum_{R \in X_{2}} f_{k}\left(\mathcal{F}_{R}\right)+\sum_{R \in X_{1}} f_{k}\left(\mathcal{F}_{R}\right) k$-faces of $\partial \mathcal{Q}$.

(ii) $F$ contains one auxiliary vertex. Then, it can consist of:

(a) a $(k-1)$-face of $\mathcal{F}_{R}, R \in X_{1}$ and vertex $y_{R}$, (e.g., a $(k-1)$-face of $\partial P_{1}$ and vertex $\left.y_{\{1\}}\right)$, or

(b) a $(k-1)$-face of $\mathcal{F}_{R}, R \in X_{2}$ and vertex $y_{R}$, (e.g., a $(k-1)$-face of $\mathcal{F}_{\{1,2\}}$ and vertex $\left.y_{\{1,2\}}\right)$, or

(c) a $(k-1)$-face of $\mathcal{F}_{S}, S \in \mathcal{X}_{1}$ and vertex $y_{R}$, where $S \subset R \in \mathcal{X}_{2}$, (e.g., a $(k-1)$-face of $\partial P_{1}$ and vertex $y_{\{1,2\}}$ or vertex $\left.y_{\{1,3\}}\right)$,

for a total of faces equal to:

$$
\begin{aligned}
\overbrace{\sum_{R \in X_{1}} f_{k-1}\left(\mathcal{F}_{R}\right)}^{\text {case (a) }}+\overbrace{\sum_{R \in X_{2}} f_{k-1}\left(\mathcal{F}_{R}\right)}^{\text {case (b) }}+\overbrace{\sum_{R \in X_{2}} \sum_{\emptyset \subset S \subset R} f_{k-1}\left(\mathcal{F}_{S}\right)}^{\text {case (c) }} \\
=\sum_{R \in X_{2}} f_{k-1}\left(\mathcal{F}_{R}\right)+3 \sum_{R \in X_{1}} f_{k-1}\left(\mathcal{F}_{R}\right) .
\end{aligned}
$$

(iii) $F$ contains two auxiliary vertices. Then, it is necessarily a $(k-2)$-face of $\mathcal{F}_{R}, R \in X_{1}$ and vertices $y_{R}$ and $y_{S}$, where $S \in X_{2}$ such that $R \subset S$, (e.g., a $(k-2)$-face of $\partial P_{1}$ and vertices $y_{\{1\}}$ and either $y_{\{1,2\}}$ or $\left.y_{\{1,3\}}\right)$, for a total of $2 \sum_{R \in X_{1}} f_{k-2}\left(\mathcal{F}_{R}\right)$ faces.

Summing over all previous cases we obtain the following relation, for all $0 \leq k \leq d+1$ :

$$
f_{k}(\partial \mathcal{Q})=f_{k}\left(\mathcal{F}_{[3]}\right)+\sum_{R \in X_{2}}\left[f_{k}\left(\mathcal{F}_{R}\right)+f_{k-1}\left(\mathcal{F}_{R}\right)\right]+\sum_{R \in X_{1}}\left[f_{k}\left(\mathcal{F}_{R}\right)+3 f_{k-1}\left(\mathcal{F}_{R}\right)+2 f_{k-2}\left(\mathcal{F}_{R}\right)\right]
$$

Relation (7) also holds for $k \in\{-1,0\}$, since, by convention, we have set $f_{l}\left(\mathcal{F}_{S}\right)=0$ for all $l<-1$ and $\emptyset \subset S \subseteq[3]$.

Denote by $\mathcal{Y}$ a generic subset of faces of $\mathcal{C}$. $\mathcal{Y}$ will either be a subcomplex of the boundary complex $\partial \mathcal{C}$ of $\mathcal{C}$, or one of the $\mathcal{F}_{R}$ 's. Let $\delta$ be the dimension of $\mathcal{Y}$. Then we can define the $h$-vector of $\mathcal{Y}$ as

$$
h_{k}(\mathcal{Y})=\sum_{i=0}^{\delta+1}(-1)^{k-i}\left(\begin{array}{c}
\delta+1-i \\
\delta+1-k
\end{array}\right) f_{i-1}(\mathcal{Y}) .
$$

Another quantity that will be heavily used in the rest of the paper is that we call the $m$-order $g$-vector of $\mathcal{Y}$, the $k$ th element of which is given by the following recursive formula:

$$
g_{k}^{(m)}(\mathcal{Y})= \begin{cases}h_{k}(\mathcal{Y}), & m=0 \\ g_{k}^{(m-1)}(\mathcal{Y})-g_{k-1}^{(m-1)}(\mathcal{Y}), & m>0\end{cases}
$$


Observe that for $m=0$ we get the $h$-vector of $\mathcal{Y}$, for $m=1$ we get the $g$-vector of $\mathcal{Y}$. In general, $\boldsymbol{g}^{(m)}(\mathcal{Y})$ is the $m$-order backward finite difference of $\boldsymbol{h}(\mathcal{Y})$, which suggests the following lemma (see Section A.1 of Appendix A for the proof):

Lemma 2. For any $k, m \geq 0$, we have:

$$
g_{k}^{(m)}(\mathcal{Y})=\sum_{i=0}^{m}(-1)^{i}\left(\begin{array}{c}
m \\
i
\end{array}\right) h_{k-i}(\mathcal{Y}) .
$$

We next define the summation operator $\mathcal{S}_{k}(\cdot ; D, \nu)$ whose action on $\mathcal{Y}$ is as follows:

$$
\mathcal{S}_{k}(\mathcal{Y} ; D, \nu)=\sum_{i=0}^{D+1}(-1)^{k-i}\left(\begin{array}{c}
D+1-i \\
D+1-k
\end{array}\right) f_{i-1-\nu}(\mathcal{Y})
$$

Regarding the action of $\mathcal{S}_{k}(\cdot ; D, \nu)$ on $\mathcal{Y}$, it is easy to verify the following (see Section A.1 of Appendix A for the proof):

Lemma 3. Let $\delta$ be the dimension of $\mathcal{Y}, \nu \geq 0, \delta \leq D$, and $D-\delta-\nu \geq 0$. Then for any $k \geq 0$ we have:

$$
\mathcal{S}_{k}(\mathcal{Y} ; D, \nu)=g_{k-\nu}^{(D-\delta-\nu)}(\mathcal{Y})
$$

In the following lemma we relate the $h$-vectors of $\mathcal{F}_{R}$ and $\mathcal{K}_{R}$ with each other, and with the $h$-vector of $\partial \mathcal{Q}$. The last among the relations proved in the following lemma can be thought of as the analogue of the Dehn-Sommerville equations for $\mathcal{F}_{[3]}$ and $\mathcal{K}_{[3]}$. These Dehn-Sommerville-like equations are used in Section 5 to obtain refined upper bounds for the values of $h_{k}\left(\mathcal{F}_{[3]}\right)$ when $k>\left\lfloor\frac{d+2}{2}\right\rfloor$ from upper bounds on $h_{k}\left(\mathcal{K}_{[3]}\right)$ when $k<\left\lfloor\frac{d+2}{2}\right\rfloor$.

Lemma 4. The following relations hold:

$$
\begin{gathered}
h_{k}\left(\mathcal{K}_{R}\right)=\sum_{\emptyset \subset S \subseteq R} g_{k}^{(|R|-|S|)}\left(\mathcal{F}_{S}\right), \quad 0 \leq k \leq d+|R|-1, \quad \emptyset \subset R \subseteq[3] . \\
h_{k}(\partial \mathcal{Q})=h_{k}\left(\mathcal{F}_{[3]}\right)+\sum_{R \in X_{2}} h_{k}\left(\mathcal{F}_{R}\right)+\sum_{R \in X_{1}}\left[h_{k}\left(\mathcal{F}_{R}\right)+h_{k-1}\left(\mathcal{F}_{R}\right)\right], \quad 0 \leq k \leq d+2 . \\
h_{d+2-k}\left(\mathcal{F}_{[3]}\right)=h_{k}\left(\mathcal{K}_{[3]}\right), \quad 0 \leq k \leq d+2 .
\end{gathered}
$$

Proof. Relation (13) follows directly from the application of the summation operator $\mathcal{S}_{k}(\cdot ;$ $d+|R|-2,0)$ to relation (5). More precisely, from (5) we get, for all $0 \leq k \leq d+|R|-1$,

$$
\mathcal{S}_{k}\left(\mathcal{K}_{R} ; d+|R|-2,0\right)=\sum_{\emptyset \subset S \subseteq R} \mathcal{S}_{k}\left(\mathcal{F}_{S} ; d+|R|-2,0\right)
$$

Relation (13) now immediately follows by noticing that: 
- By applying Lemma 3 on the left-hand-side of (16), with $\delta \leftarrow d+|R|-2, D \leftarrow d+|R|-2$ and $\nu \leftarrow 0$, we get

$$
\mathcal{S}_{k}\left(\mathcal{K}_{R} ; d+|R|-2,0\right)=g_{k-0}^{((d+|R|-2)-(d+|R|-2)-0)}\left(\mathcal{K}_{R}\right)=h_{k}\left(\mathcal{K}_{R}\right) .
$$

- Similarly, by applying Lemma 3 on the right-hand-side of (16), with $\delta \leftarrow d+|S|-2$, $D \leftarrow d+|R|-2, \nu \leftarrow 0$, we get:

$$
\mathcal{S}_{k}\left(\mathcal{F}_{S} ; d+|R|-2,0\right)=g_{k-0}^{((d+|R|-2)-(d+|S|-2)-0)}\left(\mathcal{F}_{S}\right)=g_{k}^{(|R|-|S|)}\left(\mathcal{F}_{S}\right) .
$$

To prove (14), we apply the summation operator $\mathcal{S}_{k}(\cdot ; d+1 ; 0)$ to the $(d+1)$-complex $\partial \mathcal{Q}$. Using relation $(7)$, we get, for all $0 \leq k \leq d+2$ :

$$
\begin{aligned}
\mathcal{S}_{k}(\partial \mathcal{Q} ; d+1 ; 0)= & \mathcal{S}_{k}\left(\mathcal{F}_{[3]} ; d+1 ; 0\right)+\sum_{R \in \mathcal{X}_{2}}\left[\mathcal{S}_{k}\left(\mathcal{F}_{R} ; d+1 ; 0\right)+\mathcal{S}_{k}\left(\mathcal{F}_{R} ; d+1 ; 1\right)\right] \\
& +\sum_{R \in \mathcal{X}_{1}}\left[\mathcal{S}_{k}\left(\mathcal{F}_{R} ; d+1 ; 0\right)+3 \mathcal{S}_{k}\left(\mathcal{F}_{R} ; d+1 ; 1\right)+2 \mathcal{S}_{k}\left(\mathcal{F}_{R} ; d+1 ; 2\right)\right],
\end{aligned}
$$

which, using Lemma 3, gives, for all $0 \leq k \leq d+2$ :

$g_{k}^{(0)}(\partial \mathcal{Q})=g_{k}^{(0)}\left(\mathcal{F}_{[3]}\right)+\sum_{R \in \mathcal{X}_{2}}\left[g_{k}^{(1)}\left(\mathcal{F}_{R}\right)+g_{k-1}^{(0)}\left(\mathcal{F}_{R}\right)\right]+\sum_{R \in \mathcal{X}_{1}}\left[g_{k}^{(2)}\left(\mathcal{F}_{R}\right)+3 g_{k-1}^{(1)}\left(\mathcal{F}_{R}\right)+2 g_{k-2}^{(0)}\left(\mathcal{F}_{R}\right)\right]$.

Relation (14) follows by expanding $\boldsymbol{g}^{(m)}(\cdot), 1 \leq m \leq 2$, according to Lemma 2, and gathering common terms.

To prove what we named the Dehn-Sommerville-like equations for $\mathcal{F}_{[3]}$ (cf. (15)), we replace $k$ by $d+2-k$ in (14), to get, for all $0 \leq k \leq d+2$ :

$$
h_{d+2-k}(\partial \mathcal{Q})=h_{d+2-k}\left(\mathcal{F}_{[3]}\right)+\sum_{R \in X_{2}} h_{d+2-k}\left(\mathcal{F}_{R}\right)+\sum_{R \in X_{1}}\left[h_{d+2-k}\left(\mathcal{F}_{R}\right)+h_{d+1-k}\left(\mathcal{F}_{R}\right)\right] .
$$

Using the above relation, in conjunction with (14), the Dehn-Sommerville equations for $\partial \mathcal{Q}$ become:

$$
\begin{aligned}
h_{d+2-k}\left(\mathcal{F}_{[3]}\right) & +\sum_{R \in X_{2}} h_{d+2-k}\left(\mathcal{F}_{R}\right)+\sum_{R \in X_{1}}\left[h_{d+2-k}\left(\mathcal{F}_{R}\right)+h_{d+1-k}\left(\mathcal{F}_{R}\right)\right] \\
& =h_{k}\left(\mathcal{F}_{[3]}\right)+\sum_{R \in X_{2}} h_{k}\left(\mathcal{F}_{R}\right)+\sum_{R \in X_{1}}\left[h_{k}\left(\mathcal{F}_{R}\right)+h_{k-1}\left(\mathcal{F}_{R}\right)\right] .
\end{aligned}
$$

Using the Dehn-Sommerville equations for $\mathcal{F}_{R}, R \in X_{1}$, as well as the Dehn-Sommerville-like equations for $\mathcal{F}_{R}, R \in X_{2}$ (cf. [19, rel. (3.10)]), we get:

$$
\begin{aligned}
h_{d+2-k}\left(\mathcal{F}_{[3]}\right) & +\sum_{R \in \mathcal{X}_{2}}\left[h_{k-1}\left(\mathcal{F}_{R}\right)+\sum_{\emptyset \subset S \subset R} g_{k-1}\left(\mathcal{F}_{S}\right)\right]+\sum_{R \in \mathcal{X}_{1}}\left[h_{k-2}\left(\mathcal{F}_{R}\right)+h_{k-1}\left(\mathcal{F}_{R}\right)\right] \\
& =h_{k}\left(\mathcal{F}_{[3]}\right)+\sum_{R \in \mathcal{X}_{2}} h_{k}\left(\mathcal{F}_{R}\right)+\sum_{R \in \mathcal{X}_{1}}\left[h_{k}\left(\mathcal{F}_{R}\right)+h_{k-1}\left(\mathcal{F}_{R}\right)\right] .
\end{aligned}
$$


Finally, solving in terms of $h_{d+2-k}\left(\mathcal{F}_{[3]}\right)$, we arrive at the following:

$$
\begin{aligned}
h_{d+2-k}\left(\mathcal{F}_{[3]}\right)= & h_{k}\left(\mathcal{F}_{[3]}\right)+\sum_{R \in X_{2}} h_{k}\left(\mathcal{F}_{R}\right)+\sum_{R \in X_{1}}\left[h_{k}\left(\mathcal{F}_{R}\right)+h_{k-1}\left(\mathcal{F}_{R}\right)\right] \\
& -\sum_{R \in X_{2}}\left[h_{k-1}\left(\mathcal{F}_{R}\right)+\sum_{\emptyset \subset S \subset R} g_{k-1}\left(\mathcal{F}_{S}\right)\right]-\sum_{R \in X_{1}}\left[h_{k-2}\left(\mathcal{F}_{R}\right)+h_{k-1}\left(\mathcal{F}_{R}\right)\right] \\
= & h_{k}\left(\mathcal{F}_{[3]}\right)+\sum_{R \in X_{2}} h_{k}\left(\mathcal{F}_{R}\right)+\sum_{R \in X_{1}}\left[h_{k}\left(\mathcal{F}_{R}\right)+h_{k-1}\left(\mathcal{F}_{R}\right)\right] \\
& -\sum_{R \in X_{2}} h_{k-1}\left(\mathcal{F}_{R}\right)-2 \sum_{R \in X_{1}} g_{k-1}\left(\mathcal{F}_{R}\right)-\sum_{R \in X_{1}}\left[h_{k-2}\left(\mathcal{F}_{R}\right)+h_{k-1}\left(\mathcal{F}_{R}\right)\right] \\
= & h_{k}\left(\mathcal{F}_{[3]}\right)+\sum_{R \in X_{2}}\left[h_{k}\left(\mathcal{F}_{R}\right)-h_{k-1}\left(\mathcal{F}_{R}\right)\right] \\
& +\sum_{R \in X_{1}}\left[h_{k}\left(\mathcal{F}_{R}\right)+h_{k-1}\left(\mathcal{F}_{R}\right)-2 g_{k-1}\left(\mathcal{F}_{R}\right)-h_{k-2}\left(\mathcal{F}_{R}\right)-h_{k-1}\left(\mathcal{F}_{R}\right)\right] \\
= & h_{k}\left(\mathcal{F}_{[3]}\right)+\sum_{R \in X_{2}} g_{k}\left(\mathcal{F}_{R}\right)+\sum_{R \in X_{1}}\left[h_{k}\left(\mathcal{F}_{R}\right)-2 h_{k-1}\left(\mathcal{F}_{R}\right)+h_{k-2}\left(\mathcal{F}_{R}\right)\right] \\
= & h_{k}\left(\mathcal{F}_{[3]}\right)+\sum_{R \in X_{2}} g_{k}\left(\mathcal{F}_{R}\right)+\sum_{R \in X_{1}} g_{k}^{(2)}\left(\mathcal{F}_{R}\right) \\
= & h_{k}\left(\mathcal{K}_{[3]}\right),
\end{aligned}
$$

where for the last equality we used relation (13) for $R \equiv[3]$.

\section{Recurrence relation for $h\left(\mathcal{F}_{[3]}\right)$}

Recall that we denote by $\mathcal{V}$ the vertex set of $\partial \mathcal{Q}$ and by $\mathcal{V}_{i}$ the (Cayley embedding of the) vertex set of $\partial P_{i}, 1 \leq i \leq 3$. Recall also that for a vertex $v$ of $\mathcal{Y}, \mathcal{Y} / v$ denotes the link of $v$ in $\mathcal{Y}$. McMullen [24] showed that for any $d$-dimensional polytope $P$ the following relation holds:

$$
(k+1) h_{k+1}(\partial P)+(d-k) h_{k}(\partial P)=\sum_{v \in \operatorname{vert}(\partial P)} h_{k}(\partial P / v), \quad 0 \leq k \leq d-1 .
$$

Applying relation $(18)$ to the $(d+2)$-dimensional polytope $\mathcal{Q}$, we have, for all $0 \leq$ $k \leq d+1:$

$$
(k+1) h_{k+1}(\partial \mathcal{Q})+(d+2-k) h_{k}(\partial \mathcal{Q})=\sum_{v \in \mathcal{V}} h_{k}(\partial \mathcal{Q} / v)=\sum_{v \in \mathcal{V}_{[3]}} h_{k}(\partial \mathcal{Q} / v)+\sum_{\emptyset \subset R \subset[3]} h_{k}\left(\partial \mathcal{Q} / y_{R}\right)
$$

where we used the fact that $\mathcal{V}$ is the disjoint union of the vertex sets $\mathcal{V}_{[3]}=\mathcal{V}_{1} \cup \mathcal{V}_{2} \cup \mathcal{V}_{3}$ and $\left\{y_{R} \mid \emptyset \subset R \subset[3]\right\}$. The following lemma offers convenient expressions for the elements in the sums of the right-hand side of (19) in terms of the $h$-vectors of the $\mathcal{F}_{R}$ 's and $\mathcal{K}_{R}$ 's. 
Lemma 5. The h-vectors of the complexes $\partial \mathcal{Q} / v, v \in \mathcal{V}_{i}, i=1,2,3, \partial \mathcal{Q} / y_{R}, R \in X_{1}$, and $\partial \mathcal{Q} / y_{R}, R \in \mathcal{X}_{2}$ are given by the following relations:

$$
\begin{gathered}
h_{k}(\partial \mathcal{Q} / v)=h_{k}\left(\mathcal{K}_{[3]} / v\right)+\sum_{\{i\} \subseteq R \subset[3]} h_{k-1}\left(\mathcal{K}_{R} / v\right)+h_{k-2}\left(\mathcal{K}_{\{i\}} / v\right), \quad v \in \mathcal{V}_{i}, \quad i \in[3], \\
h_{k}\left(\partial \mathcal{Q} / y_{R}\right)=h_{k}\left(\mathcal{F}_{R}\right)+h_{k-1}\left(\mathcal{F}_{R}\right), \quad R \in X_{1}, \\
h_{k}\left(\partial \mathcal{Q} / y_{R}\right)=\sum_{\emptyset \subset S \subseteq R} h_{k}\left(\mathcal{F}_{S}\right), \quad R \in X_{2} .
\end{gathered}
$$

Proof. We start by proving relation (20). Without loss of generality we assume that $v \in \mathcal{V}_{1}$; the cases $v \in \mathcal{V}_{2}$ and $v \in \mathcal{V}_{3}$ are entirely analogous.

Let $F$ be a $k$-face of $\partial \mathcal{Q} / v$. We have the following cases depending on the number of additional points $y_{R}, \emptyset \subset R \subset[3]$, that $F$ contains:

(i) $F$ does not contain any additional points. Then, it is a $k$-face of $\mathcal{K}_{[3]} / v$.

(ii) $F$ contains one additional point. Then, it can consist of:

(a) a $(k-1)$-face of $\mathcal{K}_{\{1\}} / v\left(\equiv \partial P_{1} / v\right)$ and point $y_{\{1\}}$, or

(b) a $(k-1)$-face of $\mathcal{K}_{\{1,2\}} / v$, and point $y_{\{1,2\}}$, or

(c) a $(k-1)$-face of $\mathcal{K}_{\{1,3\}} / v$, and point $y_{\{1,3\}}$.

(iii) $F$ contains two additional points. Then, it can consist of a $(k-2)$-face of $\mathcal{K}_{\{1\}} / v$ and points $y_{\{1\}}$ and $y_{\{1,2\}}$, or points $y_{\{1\}}$ and $y_{\{1,3\}}$.

Summing over all previous cases we obtain the following relation:

$$
f_{k}(\partial \mathcal{Q} / v)=\overbrace{f_{k}\left(\mathcal{K}_{[3]} / v\right)}^{\text {case (i) }}+\overbrace{\sum_{\{1\} \subseteq R \subset[3]} f_{k-1}\left(\mathcal{K}_{R} / v\right)}^{\text {case (ii) }}+\overbrace{2 f_{k-2}\left(\mathcal{K}_{\{1\}} / v\right)}^{\text {case (iii) }}, \quad v \in \mathcal{V}_{1} .
$$

We apply the summation operator $\mathcal{S}_{k}(\cdot ; d, 0)$ to the $d$-complex $\partial \mathcal{Q} / v$ and obtain:

$$
g_{k}^{(0)}(\partial \mathcal{Q} / v)=g_{k}^{(0)}\left(\mathcal{K}_{[3]} / v\right)+\sum_{\{1\} \subseteq R \subset[3]} g_{k-1}^{(2-|R|)}\left(\mathcal{K}_{R} / v\right)+2 g_{k-2}^{(0)}\left(\mathcal{K}_{\{1\}} / v\right)
$$

which finally gives, for any $v \in \mathcal{V}_{1}$ :

$$
\begin{aligned}
h_{k}(\partial \mathcal{Q} / v)= & h_{k}\left(\mathcal{K}_{[3]} / v\right)+\left(g_{k-1}\left(\mathcal{K}_{\{1\}} / v\right)+\sum_{\{1\} \subset R \subset[3]} h_{k-1}\left(\mathcal{K}_{R} / v\right)\right)+2 h_{k-2}\left(\mathcal{K}_{\{1\}} / v\right) \\
= & h_{k}\left(\mathcal{K}_{[3]} / v\right)+h_{k-1}\left(\mathcal{K}_{\{1\}} / v\right)-h_{k-2}\left(\mathcal{K}_{\{1\}} / v\right) \\
& +\sum_{\{1\} \subset R \subset[3]} h_{k-1}\left(\mathcal{K}_{R} / v\right)+2 h_{k-2}\left(\mathcal{K}_{\{1\}} / v\right)
\end{aligned}
$$




$$
=h_{k}\left(\mathcal{K}_{[3]} / v\right)+\sum_{\{1\} \subseteq R \subset[3]} h_{k-1}\left(\mathcal{K}_{R} / v\right)+h_{k-2}\left(\mathcal{K}_{\{1\}} / v\right)
$$

To prove (21) consider a $k$-face of $\partial \mathcal{Q} / y_{R}, R \in X_{1}$. Such a face is either a $k$-face of $\mathcal{F}_{R}$, or consists of a $(k-1)$-face of $\mathcal{F}_{R}$ and point $y_{S}$ for any $S \in \mathcal{X}_{2}$ such that $S \supset R$. Note that there are exactly two such points $y_{S}$. Hence:

$$
f_{k}\left(\partial \mathcal{Q} / y_{R}\right)=f_{k}\left(\mathcal{F}_{R}\right)+2 f_{k-1}\left(\mathcal{F}_{R}\right), \quad R \in X_{1}
$$

Applying the summation operator $\mathcal{S}_{k}(\cdot ; d, 0)$ to the simplicial $d$-complex $\partial \mathcal{Q} / y_{R}, R \in X_{1}$, and using relation (24) and Lemma 3 , we get, for any $R \in X_{1}$ :

$$
\begin{aligned}
h_{k}\left(\partial \mathcal{Q} / y_{R}\right) & =g_{k}^{(0)}\left(\partial \mathcal{Q} / y_{R}\right) & =\mathcal{S}_{k}\left(\partial \mathcal{Q} / y_{R} ; d, 0\right) \\
& =\mathcal{S}_{k}\left(\mathcal{F}_{R} ; d, 0\right)+2 \mathcal{S}_{k}\left(\mathcal{F}_{R} ; d, 1\right) & =g_{k}^{(1)}\left(\mathcal{F}_{R}\right)+2 g_{k-1}^{(0)}\left(\mathcal{F}_{R}\right) \\
& =h_{k}\left(\mathcal{F}_{R}\right)-h_{k-1}\left(\mathcal{F}_{R}\right)+2 h_{k-1}\left(\mathcal{F}_{R}\right) & =h_{k}\left(\mathcal{F}_{R}\right)+h_{k-1}\left(\mathcal{F}_{R}\right) .
\end{aligned}
$$

To prove (22) consider a $k$-face of $\partial \mathcal{Q} / y_{R}, R \in X_{2}$. This is either a $k$-face of $\mathcal{F}_{S}$, for any $\emptyset \subset S \subseteq R$, or consists of a $(k-1)$-face of $\mathcal{F}_{S}$ and point $y_{S}$ for any $\emptyset \subset S \subset R$. Hence, for any $R \in X_{2}$, we have:

$$
f_{k}\left(\partial \mathcal{Q} / y_{R}\right)=\sum_{\emptyset \subset S \subseteq R} f_{k}\left(\mathcal{F}_{S}\right)+\sum_{\emptyset \subset S \subset R} f_{k-1}\left(\mathcal{F}_{S}\right)=f_{k}\left(\mathcal{F}_{R}\right)+\sum_{\emptyset \subset S \subset R}\left[f_{k}\left(\mathcal{F}_{S}\right)+f_{k-1}\left(\mathcal{F}_{S}\right)\right]
$$

Applying the summation operator $\mathcal{S}_{k}(\cdot ; d, 0)$ to the $d$-dimensional complex $\partial \mathcal{Q} / y_{R}, R \in X_{2}$, and using relation (25), along with Lemma 3 , we get, for any $R \in X_{2}$ :

$$
\begin{aligned}
h_{k}\left(\partial \mathcal{Q} / y_{R}\right)=\mathcal{S}_{k}\left(\partial \mathcal{Q} / y_{R} ; d, 0\right) & =\mathcal{S}_{k}\left(\mathcal{F}_{R} ; d, 0\right)+\sum_{\emptyset \subset S \subset R}\left[\mathcal{S}_{k}\left(\mathcal{F}_{S} ; d, 0\right)+\mathcal{S}_{k}\left(\mathcal{F}_{S} ; d, 1\right)\right] \\
& =g_{k}^{(0)}\left(\mathcal{F}_{R}\right)+\sum_{\emptyset \subset S \subset R}\left[g_{k}^{(1)}\left(\mathcal{F}_{S}\right)+g_{k-1}^{(0)}\left(\mathcal{F}_{S}\right)\right] \\
& =h_{k}\left(\mathcal{F}_{R}\right)+\sum_{\emptyset \subset S \subset R} h_{k}\left(\mathcal{F}_{S}\right) \\
& =\sum_{\emptyset \subset S \subseteq R} h_{k}\left(\mathcal{F}_{S}\right) .
\end{aligned}
$$

The following two lemmas are essential in the proof of the upcoming recurrence relation in Lemma 8.

Lemma 6. The following relation holds, for all $0 \leq k \leq d+1$ :

$$
(k+1) h_{k+1}\left(\mathcal{F}_{[3]}\right)+(d+2-k) h_{k}\left(\mathcal{F}_{[3]}\right)=\sum_{\emptyset \subset R \subseteq[3]}(-1)^{3-|R|} \sum_{v \in \mathcal{V}_{R}} g_{k}^{(3-|R|)}\left(\mathcal{K}_{R} / v\right) .
$$

Sketch of proof. The complete proof may be found in Section A.2 of Appendix A. Our starting point is relation (19). We first substitute $h_{k}(\partial Q)$ and $h_{k+1}(\partial Q)$ on the left-hand side of (19) with their relevant expressions from (14). We then group the terms so that we get a sum of: 
(i) the left-hand side of (26),

(ii) $(k+1) h_{k+1}\left(\mathcal{F}_{R}\right)+(d+1-k) h_{k}\left(\mathcal{F}_{R}\right), R \in X_{2}$

(iii) $(k+1) h_{k+1}\left(\mathcal{F}_{R}\right)+(d-k) h_{k}\left(\mathcal{F}_{R}\right)$ and $k h_{k}\left(\mathcal{F}_{R}\right)+(d-k-1) h_{k-1}\left(\mathcal{F}_{R}\right)$ with $R \in X_{1}$

(iv) additional terms.

As will be described below, the intuition behind this grouping is to substitute the terms in (ii) and (iii) by sums involving quantities of the form $g_{k}^{(m)}\left(\mathcal{K}_{S} / v\right)$. These quantities will be grouped with the terms obtained from a similar expansion of the term $h_{k}(\partial \mathcal{Q} / v)$ appearing in the right-hand side of (19), yielding the right-hand side of (26).

In the proof of [19, Lemma 3.2], the sum in item (ii) above is shown to be equal ${ }^{3}$ to

$$
\sum_{i \in R} \sum_{v \in \mathcal{V}_{i}}\left[h_{k}\left(\mathcal{K}_{R} / v\right)-g_{k}\left(\mathcal{K}_{\{i\}} / v\right)\right]
$$

For (iii) we use (18) combined with the fact that for any $R \in \mathcal{X}_{1}, \mathcal{F}_{R} \equiv \partial P_{R}$. On the righthand side of $(19)$ we substitute $h_{k}(\partial \mathcal{Q} / v)$ and $h_{k}\left(\partial \mathcal{Q} / y_{R}\right)$ using the relations in Lemma 5. Finally, we equate our expansions of the left- and right-hand side of (19) and notice that the terms in (iv) and the expressions for $h_{k}\left(\partial \mathcal{Q} / y_{R}\right)$ cancel-out. Recalling that $g_{k}=h_{k}-h_{k-1}$ and $g_{k}^{(2)}=h_{k}-2 h_{k-1}+h_{k-2}$, we appropriately regroup the remaining terms to obtain the desired expression.

The last intermediate step that we need in order to derive the recurrence relation for the elements of $\boldsymbol{h}\left(\mathcal{F}_{[3]}\right)$ is to bound the right-hand side of (26) by an expression that does not involve the links $\mathcal{K}_{R} / v$. This is the subject of the following lemma.

Lemma 7. The following relation holds, for all $0 \leq k \leq d+1$ :

$$
\sum_{\emptyset \subset R \subseteq[3]}(-1)^{3-|R|} \sum_{v \in \mathcal{V}_{R}} g_{k}^{(3-|R|)}\left(\mathcal{K}_{R} / v\right) \leq \sum_{\emptyset \subset R \subseteq[3]}(-1)^{3-|R|} n_{R} g_{k}^{(3-|R|)}\left(\mathcal{K}_{R}\right)
$$

Proof. Let us first observe that, by rearranging terms, we can rewrite relation (27) as follows:

$$
\sum_{i=1}^{3} \sum_{v \in \mathcal{V}_{i}} \sum_{\{i\} \subseteq R \subseteq[3]}(-1)^{3-|R|} g_{k}^{(3-|R|)}\left(\mathcal{K}_{R} / v\right) \leq \sum_{i=1}^{3} \sum_{v \in \mathcal{V}_{i}} \sum_{\{i\} \subseteq R \subseteq[3]}(-1)^{3-|R|} g_{k}^{(3-|R|)}\left(\mathcal{K}_{R}\right) .
$$

Clearly, to show that relation (28) holds, it suffices to prove that:

$$
\sum_{\{i\} \subseteq R \subseteq[3]}(-1)^{3-|R|} g_{k}^{(3-|R|)}\left(\mathcal{K}_{R} / v\right) \leq \sum_{\{i\} \subseteq R \subseteq[3]}(-1)^{3-|R|} g_{k}^{(3-|R|)}\left(\mathcal{K}_{R}\right), \quad v \in \mathcal{V}_{i}, \quad i \in[3] .
$$

In the rest of the proof we shall prove relation (29) for $i=1$ and for any $v \in \mathcal{V}_{1}$. The cases $i=2$ and $i=3$ are entirely similar.

\footnotetext{
${ }^{3}$ The expression in [19] is written differently; it is equivalent, however, to the expression stated here.
} 
Fix a vertex $v \in \mathcal{V}_{1}$. Let $\partial \mathcal{Q}^{\prime}$ be the polytopal $(d+1)$-complex that we get by removing from $\partial \mathcal{Q}$ the faces that are incident to $y_{\{2,3\}}$ (see Fig. 3(left)). It is straightforward to see that: (1) the stars of $v$ in $\mathcal{Q}$ and $\partial \mathcal{Q}^{\prime}$ coincide (the faces incident to $y_{\{2,3\}}$ contain vertices from $\mathcal{V}_{\{2,3\}} \cup\left\{y_{\{2\}}, y_{\{3\}}\right\}$ only), and (2) $\partial \mathcal{Q}^{\prime}$ is shellable. To verify the latter consider a shelling $\mathrm{S}(\partial \mathcal{Q})$ of $\partial \mathcal{Q}$ that shells the star of $y_{\{2,3\}}$ in $\partial \mathcal{Q}$ last; the shelling order that we get by removing from $\mathrm{S}(\partial \mathcal{Q})$ the facets that are incident to $y_{\{2,3\}}$ is clearly a shelling order for $\partial \mathcal{Q}^{\prime}$. Let $\mathcal{S}_{R}, R \in\{\{1,2\},\{1,3\}\}$, be the star of $y_{R}$ in $\partial \mathcal{Q}^{\prime}$ (which actually coincides with the star of $y_{R}$ in $\partial \mathcal{Q}$ ). Let $\mathcal{X}$ denote the set of faces of $\partial \mathcal{Q}^{\prime}$ that are either faces in $\mathcal{S}_{\{1,2\}}$ or faces in $\mathcal{S}_{\{1,3\}}$, and let $\mathcal{G}$ denote the set of faces of $\partial \mathcal{Q}^{\prime}$ that are either faces in $\mathcal{F}_{[3]}$ or faces in $\mathcal{F}_{\{2,3\}}$. Notice that the sets $\mathcal{X}$ and $\mathcal{G}$ form a disjoint union of the faces in $\partial \mathcal{Q}^{\prime}$, which implies that:

$$
f_{k}\left(\partial \mathcal{Q}^{\prime}\right)=f_{k}(\mathcal{X})+f_{k}(\mathcal{G}), \quad-1 \leq k \leq d+1 .
$$

Notice that $\mathcal{X}$ is a $(d+1)$-complex, whereas $\mathcal{G}$ is a set of faces with maximal dimension $d+1$. By applying the summation operator $\mathcal{S}_{k}(\cdot ; d+1,0)$ to $(30)$, we immediately get the corresponding $h$-vector relation:

$$
h_{k}\left(\partial \mathcal{Q}^{\prime}\right)=h_{k}(\mathcal{X})+h_{k}(\mathcal{G}), \quad 0 \leq k \leq d+2 .
$$

We claim that there exists a specific shelling $\mathbf{S}\left(\partial \mathcal{Q}^{\prime}\right)$ of $\partial \mathcal{Q}^{\prime}$, which is actually an initial segment of a shelling of $\partial \mathcal{Q}$ that shells the star of $y_{\{2,3\}}$ last, with the property that the corresponding shelling order has the facets in $\mathcal{X}$ before the facets in $\mathcal{G}$. We postpone the proof of this claim, and assume for now that the claim holds true.

The same argumentation can be applied to the links of vertices $v \in \mathcal{V}_{1}: \partial \mathcal{Q}^{\prime} / v$ can be seen as the disjoint union of the sets $\mathcal{X} / v$ and $\mathcal{G} / v$, while the particular shelling $\mathrm{S}\left(\partial \mathcal{Q}^{\prime}\right)$
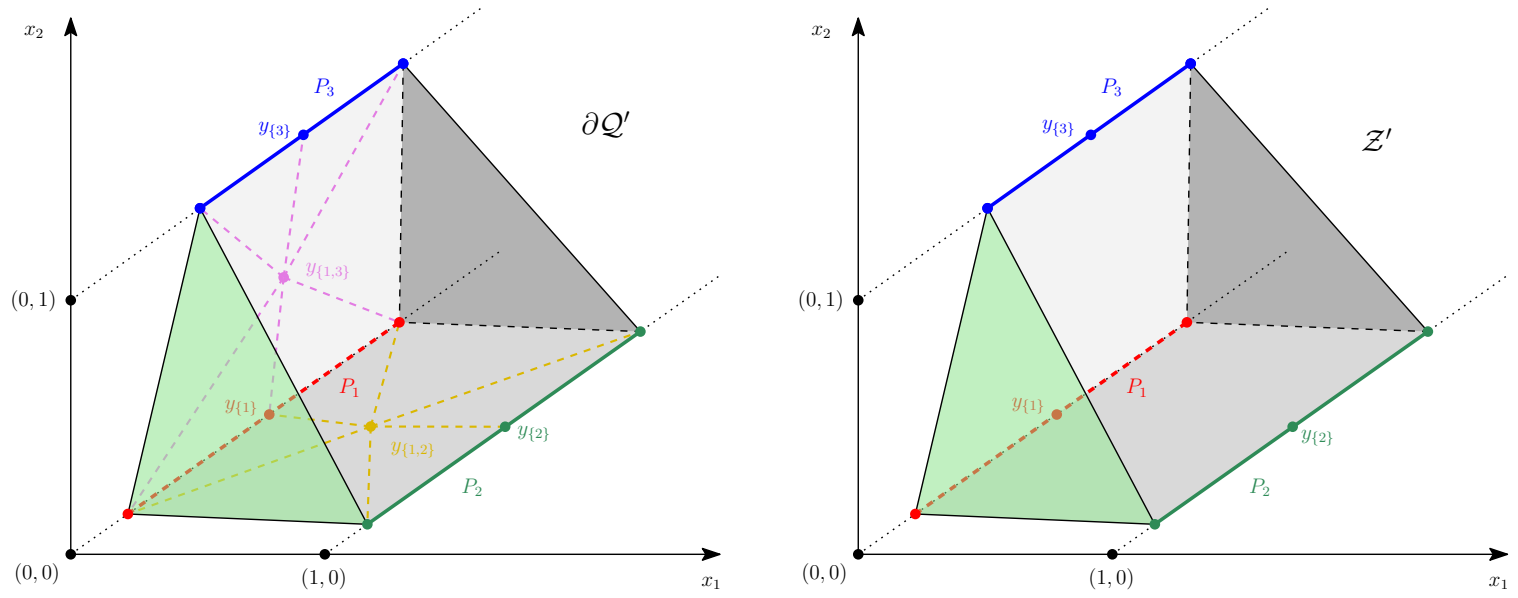

Figure 3: Left: the $(d+1)$-complex $\partial \mathcal{Q}^{\prime}$ that we get from $\partial \mathcal{Q}$ be removing all faces incident to $y_{\{2,3\}}$. Right: the $(d+1)$-complex $\mathcal{Z}^{\prime}$ that we get from the Cayley polytope $\mathcal{C}_{[3]}$ of $P_{1}, P_{2}$ and $P_{3}$, after we: (i) have performed stellar subdivisions using the vertices $y_{\{1\}}, y_{\{2\}}$ and $y_{\{3\}}$ (which yields the $\left(d+1\right.$ )-polytope $\mathcal{Z}$ ), and (ii) have removed the facet $\mathcal{Q}_{\{2,3\}}$ from $\mathcal{Z}$. 
of $\partial \mathcal{Q}^{\prime}$ that shells $\mathcal{X}$ first, induces a particular shelling $\mathrm{S}\left(\partial \mathcal{Q}^{\prime} / v\right)$ for $\partial \mathcal{Q}^{\prime} / v$ that shells the facets of $\partial \mathcal{Q}^{\prime} / v$ in $\mathcal{X} / v$ first. From these observations we immediately arrive at the following $h$-vector relation for $\partial \mathcal{Q}^{\prime} / v, \mathcal{X} / v$ and $\mathcal{G} / v$ :

$$
h_{k}\left(\partial \mathcal{Q}^{\prime} / v\right)=h_{k}(\mathcal{X} / v)+h_{k}(\mathcal{G} / v), \quad 0 \leq k \leq d+1 .
$$

Let us now consider the dual graph $G^{\Delta}(\partial \mathcal{Q})$ of $\partial \mathcal{Q}$, oriented according to the shelling $\mathrm{S}(\partial \mathcal{Q})$, as well as the dual graph $G^{\Delta}(\partial \mathcal{Q} / v)$ of $\partial \mathcal{Q} / v$, also oriented according to the shelling $\mathrm{S}(\partial \mathcal{Q} / v)$. We will denote by $\mathcal{V}^{\Delta}(\mathcal{Y})$ the subset of vertices of $G^{\Delta}(\partial \mathcal{Q})$ that are the duals of the facets in $\partial \mathcal{Q}$ that belong to $\mathcal{Y}$, where $\mathcal{Y}$ stands for a subset of the set of faces of $\partial \mathcal{Q}$.

Since $\mathrm{S}(\partial \mathcal{Q} / v)$ is induced from $\mathrm{S}(\partial \mathcal{Q}), G^{\Delta}(\partial \mathcal{Q} / v)$ is isomorphic to the subgraph of $G^{\Delta}(\partial \mathcal{Q})$ defined over $\mathcal{V}^{\Delta}(\operatorname{star}(v, \partial \mathcal{Q}))$. Moreover, $h_{k}(\partial \mathcal{Q})$ counts the number of vertices of $\mathcal{V}^{\Delta}(\partial \mathcal{Q})$ with in-degree equal to $k$, while $h_{k}(\mathcal{G})$ counts the number of vertices of $\mathcal{V}^{\Delta}(\mathcal{G})$ of in-degree $k$ in $G^{\Delta}(\partial \mathcal{Q})$, because the shelling we have chosen shells first all facets of $\mathcal{X}$ before shelling the remaining facets of $\partial \mathcal{Q}^{\prime}$. Consequently, $h_{k}(\mathcal{G})$ counts the number of vertices of $\mathcal{V}^{\Delta}(\mathcal{G})$ of in-degree $k$ in $G^{\Delta}(\partial \mathcal{Q})$; in an analogous manner, we can conclude that $h_{k}(\mathcal{G} / v)$ counts the number of vertices of $\mathcal{V}^{\Delta}(\operatorname{star}(v, \mathcal{G}))$ with in-degree $k$ in $G^{\Delta}(\partial \mathcal{Q} / v)$. Since, however, $G^{\Delta}(\partial \mathcal{Q} / v)$ is the subgraph of $G^{\Delta}(\partial \mathcal{Q})$ that corresponds to the face $v^{\Delta}$ of $G^{\Delta}(\partial \mathcal{Q})$, the number of vertices of $\mathcal{V}^{\Delta}(\operatorname{star}(v, \mathcal{G}))$ with in-degree $k$ cannot exceed the number of vertices of $\mathcal{V}^{\Delta}(\mathcal{G})$ with in-degree $k$. Hence,

$$
h_{k}(\mathcal{G} / v) \leq h_{k}(\mathcal{G}), \quad 0 \leq k \leq d+2 .
$$

On the other hand, recall that $\mathcal{G}$ is the disjoint union of $\mathcal{F}_{[3]}$ and $\mathcal{F}_{\{2,3\}}$. Using expressions (6), in conjunction with the fact that $\mathcal{F}_{S} \equiv \mathcal{K}_{S}$ for $S \in \mathcal{X}_{1}$, we have, for all $-1 \leq k \leq d+1$ :

$$
\begin{aligned}
f_{k}(\mathcal{G}) & =f_{k}\left(\mathcal{F}_{[3]}\right)+f_{k}\left(\mathcal{F}_{\{2,3\}}\right) \\
& =\sum_{\emptyset \subset R \subseteq[3]}(-1)^{3-|R|} f_{k}\left(\mathcal{K}_{R}\right)+\sum_{\emptyset \subset R \subseteq\{2,3\}}(-1)^{2-|R|} f_{k}\left(\mathcal{K}_{R}\right) \\
& =\sum_{\emptyset \subset R \subseteq[3]}(-1)^{3-|R|} f_{k}\left(\mathcal{K}_{R}\right)-\sum_{\emptyset \subset R \subseteq\{2,3\}}(-1)^{3-|R|} f_{k}\left(\mathcal{K}_{R}\right) \\
& =\sum_{\{1\} \subseteq R \subseteq[3]}(-1)^{3-|R|} f_{k}\left(\mathcal{K}_{R}\right) .
\end{aligned}
$$

By a similar argument, we can arrive at the following expression for $f_{k}(\mathcal{G} / v)$ :

$$
f_{k}(\mathcal{G} / v)=\sum_{\{1\} \subseteq R \subseteq[3]}(-1)^{3-|R|} f_{k}\left(\mathcal{K}_{R} / v\right), \quad-1 \leq k \leq d .
$$

By applying the summation operators $\mathcal{S}_{k}(\cdot ; d+1,0)$ and $\mathcal{S}_{k}(\cdot ; d, 0)$ to relations $(34)$ and $(35)$, respectively, we get the corresponding $h$-vector relations:

$$
\begin{aligned}
h_{k}(\mathcal{G}) & =\sum_{\{1\} \subseteq R \subseteq[3]}(-1)^{3-|R|} g_{k}^{(3-|R|)}\left(\mathcal{K}_{R}\right), \quad 0 \leq k \leq d+2, \\
h_{k}(\mathcal{G} / v) & =\sum_{\{1\} \subseteq R \subseteq[3]}(-1)^{3-|R|} g_{k}^{(3-|R|)}\left(\mathcal{K}_{R} / v\right), \quad 0 \leq k \leq d+1 .
\end{aligned}
$$


Relation (29) (for $i=1$ ) follows by substituting the expressions for $h_{k}(\mathcal{G})$ and $h_{k}(\mathcal{G} / v)$ from (36) in (33).

To finish our proof, it remains to establish our claim that there exists a specific shelling $\mathrm{S}\left(\partial \mathcal{Q}^{\prime}\right)$ of $\partial \mathcal{Q}^{\prime}$ with the property that the facets of $\mathcal{X}$ appear in the shelling before the facets of $\mathcal{G}$. Let us start with some definitions: we denote by $\mathcal{Z}$ the $(d+1)$-complex we get by performing the stellar subdivisions on $\mathcal{C}_{[3]}$ using the vertices $y_{R}, R \in X_{1}$ (see also Fig. 3(right)), and by $\mathcal{Q}_{R}, R \in X_{2}$ the $(d+1)$-complex that we get by performing stellar subdivisions on the non-simplicial proper faces of $\mathcal{C}_{R}$, namely the faces $\mathcal{C}_{S}, \emptyset \subset S \subset R$. Notice that $\mathcal{Q}_{R}, R \in X_{2}$, is nothing but a facet of $\mathcal{Z}$, while $\partial \mathcal{Q}_{R}$ is actually the link of $y_{R}$ in $\partial \mathcal{Q}$. In fact, we can separate the facets of $\mathcal{Z}$ in two categories; they are either (1) facets of the form $\mathcal{Q}_{R}, R \in \mathcal{X}_{2}$, which are non-simplicial, or (2) facets in $\mathcal{G}$ (or $\mathcal{F}_{[3]}$ ), which are simplicial. Moreover, notice that $\operatorname{star}\left(y_{R}, \mathcal{Z}\right), R \in X_{1}$, consists of the faces belonging to the two facets $\mathcal{Q}_{S}, R \subset S \subset[3]$ of $\mathcal{Z}$. Since stellar subdivisions produce polytopal complexes [6], $\mathcal{Z}$ is polytopal and, thus, shellable. In fact, there exists a particular (line) shelling $\mathrm{S}(\mathcal{Z})$ of $\mathcal{Z}$ in which the facets of $\operatorname{star}\left(y_{\{1\}}, \mathcal{Z}\right)$ appear first, while $\mathcal{Q}_{\{2,3\}}$ is the last facet in $\mathrm{S}(\mathcal{Z})$. More precisely, for this particular shelling of $\mathcal{Z}$, the two facets $\mathcal{Q}_{\{1,2\}}$ and $\mathcal{Q}_{\{1,3\}}$ appear first, followed by the facets in $\mathcal{G}$, which, in turn, are followed by the facet $\mathcal{Q}_{\{2,3\}}$.

Let us call $\mathcal{Z}^{\prime}$ the $(d+1)$-complex we get by removing $\mathcal{Q}_{\{2,3\}}$ from $\mathcal{Z}$. The complex $\mathcal{Z}^{\prime}$ is shellable (it follows from the fact that $\mathrm{S}(\mathcal{Z})$ has $\mathcal{Q}_{\{2,3\}}$ as its last facet), while the particular line shelling $\mathrm{S}(\mathcal{Z})$ of $\mathcal{Z}$ described above, yields a shelling $\mathrm{S}\left(\mathcal{Z}^{\prime}\right)$ for $\mathcal{Z}^{\prime}$ in which the facets $\mathcal{Q}_{\{1,2\}}$ and $\mathcal{Q}_{\{1,3\}}$ appear first, followed by the facets in $\mathcal{G}$. Notice that if we perform stellar subdivisions on the two non-simplicial facets $\mathcal{Q}_{\{1,2\}}$ and $\mathcal{Q}_{\{1,3\}}$ of $\mathcal{Z}^{\prime}$ (using the vertices $y_{\{1,2\}}$ and $\left.y_{\{1,3\}}\right)$, we arrive at the simplicial $(d+1)$-complex $\partial \mathcal{Q}^{\prime}$ described earlier. Furthermore, from the particular shelling $\mathrm{S}\left(\mathcal{Z}^{\prime}\right)$ of $\mathcal{Z}^{\prime}$ described above, we obtain the soughtfor shelling for $\partial \mathcal{Q}^{\prime}$ that shells $\mathcal{X}$ first and $\mathcal{G}$ last. To see this, notice that given any shelling order for $\partial P_{i}, i=1,2,3$, we may construct a shelling for $\mathcal{Q}_{R}, R \in\{\{1,2\},\{1,3\}\}$, that: (1) shells $\operatorname{st}\left(y_{\{1\}}, \mathcal{Q}_{R}\right)$ first, (2) shells $\operatorname{st}\left(y_{R \backslash\{1\}}, \mathcal{Q}_{R}\right)$ last, and (3) the shelling order of the facets in both stars is the order implied by the shellings of the boundary complexes $\partial P_{1}$ and $\partial P_{R \backslash\{1\}}$. This implies that if we choose shelling orders for $\partial \mathcal{Q}_{\{1,2\}}$ and $\partial \mathcal{Q}_{\{1,3\}}$ that respect a common shelling order for $\partial P_{1}$, we can replace the facets $\mathcal{Q}_{\{1,2\}}$ and $\mathcal{Q}_{\{1,3\}}$ in $\mathrm{S}\left(\mathcal{Z}^{\prime}\right)$ by the facets in $\operatorname{star}\left(y_{\{1,2\}}, \partial \mathcal{Q}^{\prime}\right)$ and $\operatorname{star}\left(y_{\{1,3\}}, \partial \mathcal{Q}^{\prime}\right)$, respectively, (the shelling orders of $\partial \mathcal{Q}_{\{1,2\}}$ and $\partial \mathcal{Q}_{\{1,3\}}$ are "inherited" in the shelling orders for $\operatorname{star}\left(y_{\{1,2\}}, \partial \mathcal{Q}^{\prime}\right)$ and $\left.\operatorname{star}\left(y_{\{1,3\}}, \partial \mathcal{Q}^{\prime}\right)\right)$ and arrive at a shelling order for $\partial \mathcal{Q}^{\prime}$ with the desired property.

Using inequality (27) in Lemma 7, we arrive at the following recurrence relation for the elements of $\boldsymbol{h}\left(\mathcal{F}_{[3]}\right)$; its proof may be found in Section A.2 in Appendix A. The recurrence relation in the following lemma is used in Lemma 10 (see upcoming section) to get an upper bound on $h_{k}\left(\mathcal{F}_{[3]}\right)$ for all $0 \leq k \leq d+2$.

Lemma 8. For all $0 \leq k \leq d+1$, we have:

$$
h_{k+1}\left(\mathcal{F}_{[3]}\right) \leq \frac{n_{[3]}-d-2+k}{k+1} h_{k}\left(\mathcal{F}_{[3]}\right)+\sum_{i=1}^{3} \frac{n_{i}}{k+1} g_{k}\left(\mathcal{F}_{[3] \backslash\{i\}}\right) .
$$

Sketch of proof. Using Lemma 7, we can bound the left hand side of relation (26) by the 
right hand side of relation (27), which involves $g$-vectors, of various orders, of the complexes $\mathcal{K}_{R}$, where $\emptyset \subset R \subseteq[3]$. These can be substituted by their equal values from relation (13) with $R=$ [3] and for all $R \in X_{2}$. This gives an inequality involving $h$-vectors and $g$-vectors of $\mathcal{F}_{[3]}$ and $\mathcal{F}_{R}, R \in \mathcal{X}_{2}$, which simplifies to relation (37).

\section{Upper bounds}

In this section we establish upper bounds for the number of $(k+2)$-faces of $\mathcal{F}_{[3]}, 0 \leq k \leq d-1$, which immediately yield upper bounds for the number of $k$-faces of $P_{1}+P_{2}+P_{3}$. Our starting point is the recurrence relation (37). Using this recurrence relation, along with the corresponding relation for $\boldsymbol{h}\left(\mathcal{F}_{R}\right), R \in \mathcal{X}_{2}$ (cf. [19, Lemma 3.2]), we can derive the upper bounds for $\boldsymbol{h}\left(\mathcal{F}_{[3]}\right)$ and $\boldsymbol{h}\left(\mathcal{K}_{[3]}\right)$ stated in the following lemmas, as well as necessary and sufficient conditions for these bounds to be tight. These conditions will be exploited in Section 6 in order to prove the tightness of our upper bounds.

The general idea and techniques we use in the proofs of this section are parallel to those used in [19, Section 3] for the Minkowski sum of two polytopes. In particular, we utilize the recurrence relation for the $h$-vector of $\mathcal{F}_{R}, R \in X_{2}$, from [19, Lemma 3.2]:

$$
h_{k+1}\left(\mathcal{F}_{R}\right) \leq \frac{n_{R}-d-1+k}{k+1} h_{k}\left(\mathcal{F}_{R}\right)+\sum_{\emptyset \subset S \subset R} \frac{n_{R \backslash S}}{k+1} g_{k}\left(\mathcal{F}_{S}\right),
$$

as well as the upper bound for the $h$-vector of $\mathcal{F}_{R}, R \in X_{2}$, obtained from the above recurrence relation (cf. [19, Lemma 3.3]):

$$
h_{k}\left(\mathcal{F}_{R}\right) \leq \sum_{\emptyset \subset S \subseteq R}(-1)^{|S|}\left(\begin{array}{c}
n_{S}-d-2+k \\
k
\end{array}\right) .
$$

Furthermore, we apply the well-known upper bounds for the $h$ - and $g$-vector of polytopes (cf. [31, Lemma 8.26 and Corollary 8.38]):

$$
h_{k}\left(\mathcal{F}_{\{i\}}\right)=h_{k}\left(\partial P_{i}\right) \leq\left(\begin{array}{c}
n_{i}-d-1+k \\
k
\end{array}\right), \quad 0 \leq k \leq d,
$$

and

$$
g_{k}\left(\mathcal{F}_{\{i\}}\right)=g_{k}\left(\partial P_{i}\right) \leq\left(\begin{array}{c}
n_{i}-d-2+k \\
k
\end{array}\right), \quad 0 \leq k \leq d+1 .
$$

We first prove a lemma that establishes bounds for the $g$-vector of $\mathcal{F}_{R}, R \in X_{2}$.

Lemma 9. Let $R$ be a subset of [3] of cardinality 2. Then, for all $0 \leq k \leq d+2$, we have:

$$
g_{k}\left(\mathcal{F}_{R}\right) \leq \sum_{\emptyset \subset S \subseteq R}(-1)^{|S|}\left(\begin{array}{c}
n_{S}-d-3+k \\
k
\end{array}\right) .
$$

Equality holds for some $k$, where $0 \leq k \leq\left\lfloor\frac{d+1}{2}\right\rfloor$, if and only if

$$
f_{l-1}\left(\mathcal{F}_{R}\right)=\sum_{\emptyset \subset S \subseteq R}(-1)^{|S|}\left(\begin{array}{c}
n_{S} \\
l
\end{array}\right),
$$

for all $0 \leq l \leq k$. 
Proof. The bound clearly holds, as equality, for $k=0$. Indeed, for $k=0$, and recalling our convention $f_{0}\left(\mathcal{F}_{R}\right)=(-1)^{|R|-1}$, we have:

$$
h_{0}\left(\mathcal{F}_{R}\right)=\sum_{i=0}^{(d-|R|-2)+1}(-1)^{0-i}\left(\begin{array}{l}
(d-|R|-2)+1-i \\
(d-|R|-2)+1-0
\end{array}\right) f_{i-1}\left(\mathcal{F}_{R}\right)=f_{-1}\left(\mathcal{F}_{R}\right)=(-1)^{|R|-1} .
$$

Hence,

$$
\begin{aligned}
g_{0}\left(\mathcal{F}_{R}\right) & =h_{0}\left(\mathcal{F}_{R}\right)-h_{-1}\left(\mathcal{F}_{R}\right)=(-1)^{|R|-1}-0=-1=\sum_{i \in R}(-1)+1 \\
& =\sum_{i \in R}(-1)^{|\{i\}|}\left(\begin{array}{c}
n_{i}-d-3+0 \\
0
\end{array}\right)+(-1)^{|R|}\left(\begin{array}{c}
n_{R}-d-3+0 \\
0
\end{array}\right)=\sum_{\emptyset \subset S \subseteq R}(-1)^{|S|}\left(\begin{array}{c}
n_{S}-d-3+0 \\
0
\end{array}\right) .
\end{aligned}
$$

For $k \geq 1$, from [19, Lemma 3.2] we have:

$$
h_{k}\left(\mathcal{F}_{R}\right) \leq \frac{n_{R}-d-2+k}{k} h_{k-1}\left(\mathcal{F}_{R}\right)+\sum_{\emptyset \subset S \subset R} \frac{n_{R \backslash S}}{k} g_{k-1}\left(\mathcal{F}_{S}\right) .
$$

Subtracting $h_{k-1}\left(\mathcal{F}_{R}\right)$ from both sides of (39) we get:

$$
g_{k}\left(\mathcal{F}_{R}\right) \leq \frac{n_{R}-d-2}{k} h_{k-1}\left(\mathcal{F}_{R}\right)+\sum_{\emptyset \subset S \subset R} \frac{n_{R \backslash S}}{k} g_{k-1}\left(\mathcal{F}_{S}\right)
$$

Using now the upper bounds for $h_{k-1}\left(\mathcal{F}_{R}\right), g_{k-1}\left(\mathcal{F}_{S}\right), \emptyset \subset S \subset R$, and noting that $n_{R}-$ $d-2 \geq 2(d+1)-d-2=d>0$, we deduce, for any $k \geq 1$ :

$$
\begin{aligned}
g_{k}\left(\mathcal{F}_{R}\right) & \leq \frac{n_{R}-d-2}{k} \sum_{\emptyset \subset S \subseteq R}(-1)^{|S|}\left(\begin{array}{c}
n_{S}-d-3+k \\
k-1
\end{array}\right)+\sum_{\emptyset \subset S \subset R} \frac{n_{R \backslash S}}{k}\left(\begin{array}{c}
n_{S}-d-3+k \\
k-1
\end{array}\right) \\
& =\frac{n_{R}-d-2}{k}\left(\begin{array}{c}
n_{R}-d-3+k \\
k-1
\end{array}\right)-\sum_{\emptyset \subset S \subset R} \frac{n_{R}-d-2}{k}\left(\begin{array}{c}
n_{S}-d-3+k \\
k-1
\end{array}\right)+\sum_{\emptyset \subset S \subset R} \frac{n_{R \backslash S}}{k}\left(\begin{array}{c}
n_{S}-d-3+k \\
k-1
\end{array}\right) \\
& =\frac{n_{R}-d-2+k}{k}\left(\begin{array}{c}
n_{R}-d-3+k \\
k-1
\end{array}\right)-\left(\begin{array}{c}
n_{R}-d-3+k \\
k-1
\end{array}\right)-\sum_{\emptyset \subset S \subset R} \frac{n_{R}-d-2-n_{R \backslash S}}{k}\left(\begin{array}{c}
n_{S}-d-3+k \\
k-1
\end{array}\right) \\
& =\left(\begin{array}{c}
n_{R}-d-2+k \\
k
\end{array}\right)-\left(\begin{array}{c}
n_{R}-d-3+k \\
k-1
\end{array}\right)-\sum_{\emptyset \subset S \subset R} \frac{n_{S}-d-2}{k}\left(\begin{array}{c}
n_{S}-d-3+k \\
k-1
\end{array}\right) \\
& =\left(\begin{array}{c}
n_{R}-d-3+k \\
k
\end{array}\right)-\sum_{\emptyset \subset S \subset R}\left[\frac{n_{S}-d-2+k}{k}\left(\begin{array}{c}
n_{S}-d-3+k \\
k-1
\end{array}\right)-\left(\begin{array}{c}
n_{S}-d-3+k \\
k-1
\end{array}\right)\right] \\
& \left.=\left(\begin{array}{c}
n_{R}-d-3+k \\
k
\end{array}\right)-\sum_{\emptyset \subset S \subset R}\left[\begin{array}{c}
n_{S}-d-2+k \\
k
\end{array}\right)-\left(\begin{array}{c}
n_{S}-d-3+k \\
k-1
\end{array}\right)\right] \\
= & \left(\begin{array}{c}
n_{R}-d-3+k \\
k
\end{array}\right)-\sum_{\emptyset \subset S \subset R}\left(\begin{array}{c}
n_{S}-d-3+k \\
k
\end{array}\right) \\
= & \sum_{\emptyset \subset S \subseteq R}(-1)^{|S|}\left(\begin{array}{l}
n_{S}-d-3+k \\
k
\end{array}\right) .
\end{aligned}
$$

We focus now on the equality claim. Suppose first that

$$
f_{l-1}\left(\mathcal{F}_{R}\right)=\sum_{\emptyset \subset S \subseteq R}(-1)^{|S|}\left(\begin{array}{c}
n_{S} \\
l
\end{array}\right)
$$


for all $0 \leq l \leq k$. Then, by [19, Lemma 3.3], $h_{\lambda}\left(\mathcal{F}_{R}\right)=\sum_{\emptyset \subset S \subseteq R}(-1)^{|S|}\left(\begin{array}{c}n_{S}-d-2+\lambda \\ \lambda\end{array}\right)$, for $\lambda=k-1, k$, which gives:

$$
\begin{aligned}
g_{k}\left(\mathcal{F}_{R}\right) & =h_{k}\left(\mathcal{F}_{R}\right)-h_{k-1}\left(\mathcal{F}_{R}\right)=\sum_{\emptyset \subset S \subseteq R}(-1)^{|S|}\left(\begin{array}{c}
n_{S}-d-2+k \\
k
\end{array}\right)-\sum_{\emptyset \subset S \subseteq R}(-1)^{|S|}\left(\begin{array}{c}
n_{S}-d-2+k-1 \\
k-1
\end{array}\right) \\
& =\sum_{\emptyset \subset S \subseteq R}(-1)^{|S|}\left[\left(\begin{array}{c}
n_{S}-d-2+k \\
k
\end{array}\right)-\left(\begin{array}{c}
n_{S}-d-2+k-1 \\
k-1
\end{array}\right)\right]=\sum_{\emptyset \subset S \subseteq R}(-1)^{|S|}\left(\begin{array}{c}
n_{S}-d-3+k \\
k
\end{array}\right) .
\end{aligned}
$$

Suppose now that $g_{k}\left(\mathcal{F}_{R}\right)=\sum_{\emptyset \subset S \subseteq R}(-1)^{|S|}\left(\begin{array}{c}n_{S}-d-3+k \\ k\end{array}\right)$. By relation (40), we conclude that $h_{k-1}\left(\mathcal{F}_{R}\right)$ must be equal to its upper bound (cf. [19, Lemma 3.3]), since, otherwise, $g_{k}\left(\mathcal{F}_{R}\right)$ would not be maximal, which contradicts our assumption on the value of $g_{k}\left(\mathcal{F}_{R}\right)$. This gives:

$$
\begin{aligned}
h_{k}\left(\mathcal{F}_{R}\right) & =g_{k}\left(\mathcal{F}_{R}\right)+h_{k-1}\left(\mathcal{F}_{R}\right)=\sum_{\emptyset \subset S \subseteq R}(-1)^{|S|}\left(\begin{array}{c}
n_{S}-d-3+k \\
k
\end{array}\right)+\sum_{\emptyset \subset S \subseteq R}(-1)^{|S|}\left(\begin{array}{c}
n_{S}-d-2+k-1 \\
k-1
\end{array}\right) \\
& =\sum_{\emptyset \subset S \subseteq R}(-1)^{|S|}\left[\left(\begin{array}{c}
n_{S}-d-2+k-1 \\
k
\end{array}\right)+\left(\begin{array}{c}
n_{S}-d-2+k-1 \\
k-1
\end{array}\right)\right]=\sum_{\emptyset \subset S \subseteq R}(-1)^{|S|}\left(\begin{array}{c}
n_{S}-d-2+k \\
k
\end{array}\right) .
\end{aligned}
$$

Now the fact that $h_{k}\left(\mathcal{F}_{R}\right)$ is maximal, implies that $h_{l}\left(\mathcal{F}_{R}\right)$ must be equal to its maximal value for all $0 \leq l<k$. To see this, suppose that $h_{l}\left(\mathcal{F}_{R}\right)$ is not maximal for some $l$, with $0 \leq l<k$, and among all such $l$ choose the largest one. Then, Lemmas 3.2 and 3.3 in [19] imply that $h_{l+1}\left(\mathcal{F}_{R}\right)$ cannot be maximal, which contradicts the maximality of $l$. Summarizing, we deduce that if $g_{k}\left(\mathcal{F}_{R}\right)$ is equal to its upper bound in $(38)$, so is $h_{l}\left(\mathcal{F}_{R}\right)$ for all $0 \leq l \leq k$. By Lemma 3.3 in [19], this implies that $f_{l-1}\left(\mathcal{F}_{R}\right)=\sum_{\emptyset \subset S \subseteq R}(-1)^{|S|}\left(\begin{array}{c}n_{S} \\ l\end{array}\right)$, for all $0 \leq l \leq k$.

Lemma 10. For all $0 \leq k \leq d+2$, we have:

$$
h_{k}\left(\mathcal{F}_{[3]}\right) \leq \sum_{\emptyset \subset S \subseteq[3]}(-1)^{3-|S|}\left(\begin{array}{c}
n_{S}-d-3+k \\
k
\end{array}\right), \quad n_{S}=\sum_{i \in S} n_{i} .
$$

Equality holds for some $0 \leq k \leq\left\lfloor\frac{d+2}{2}\right\rfloor$, if and only if $f_{l-1}\left(\mathcal{F}_{[3]}\right)=\sum_{\emptyset \subset S \subseteq[3]}(-1)^{3-|S|}\left(\begin{array}{c}n_{S} \\ l\end{array}\right)$, for all $0 \leq l \leq k$.

Proof. We are going to prove relation (41) by induction on $k$. The result clearly holds for $k=0$, since

$$
h_{0}\left(\mathcal{F}_{[3]}\right)=1=1-3+3=\left(\begin{array}{c}
n_{[3]}-d-3 \\
0
\end{array}\right)-\sum_{i=1}^{3}\left(\begin{array}{c}
n_{[3] \backslash\{i\}}-d-3 \\
0
\end{array}\right)+\sum_{i=1}^{3}\left(\begin{array}{c}
n_{i}-d-3 \\
0
\end{array}\right) .
$$

Suppose the bound holds for some $k \geq 0$. We will show that it holds for $k+1$. Using relation (37), Lemma 9 , and the fact that, for any $k \geq 0, n_{[3]}-d-2+k \geq 3(d+1)-d-2=$ $2 d+1>0$, we have:

$$
h_{k+1}\left(\mathcal{F}_{[3]}\right) \leq \frac{n_{[3]}-d-2+k}{k+1} h_{k}\left(\mathcal{F}_{[3]}\right)+\sum_{i=1}^{3} \frac{n_{i}}{k+1} g_{k}\left(\mathcal{F}_{[3] \backslash\{i\}}\right)
$$




$$
\begin{aligned}
& \leq \frac{n_{[3]}-d-2+k}{k+1} \sum_{\emptyset \subset S \subseteq[3]}(-1)^{3-|S|}\left(\begin{array}{c}
n_{S}-d-3+k \\
k
\end{array}\right)+\sum_{i=1}^{3} \frac{n_{i}}{k+1} \sum_{\emptyset \subset S \subseteq[3] \backslash\{i\}}(-1)^{|S|}\left(\begin{array}{c}
n_{S}-d-3+k \\
k
\end{array}\right) \\
& =\frac{n_{[3]}-d-2+k}{k+1}\left(\begin{array}{c}
n_{[3]}-d-3+k \\
k
\end{array}\right)-\sum_{i=1}^{3} \frac{n_{[3]}-d-2+k}{k+1}\left(\begin{array}{c}
n_{[3] \backslash\{i\}}-d-3+k \\
k
\end{array}\right) \\
& +\sum_{i=1}^{3} \frac{n_{[3]}-d-2+k}{k+1}\left(\begin{array}{c}
n_{i}-d-3+k \\
k
\end{array}\right)+\sum_{i=1}^{3} \frac{n_{i}}{k+1}\left(\begin{array}{c}
n_{[3] \backslash\{i\}}-d-3+k \\
k
\end{array}\right) \\
& -\sum_{i=1}^{3} \frac{n_{i}}{k+1} \sum_{j \in[3] \backslash\{i\}}\left(\begin{array}{c}
n_{j}-d-3+k \\
k
\end{array}\right) \\
& =\left(\begin{array}{c}
n_{[3]}-d-2+k \\
k+1
\end{array}\right)-\sum_{i=1}^{3} \frac{n_{[3]}-d-2+k-n_{i}}{k+1}\left(\begin{array}{c}
n_{[3] \backslash\{i\}}-d-3+k \\
k
\end{array}\right) \\
& +\sum_{i=1}^{3} \frac{n_{[3]}-d-2+k-n_{[3] \backslash\{i\}}}{k+1}\left(\begin{array}{c}
n_{i}-d-3+k \\
k
\end{array}\right) \\
& =\left(\begin{array}{c}
n_{[3]}-d-2+k \\
k+1
\end{array}\right)-\sum_{i=1}^{3} \frac{n_{[3] \backslash\{i\}}-d-2+k}{k+1}\left(\begin{array}{c}
n_{[3] \backslash\{i\}}-d-3+k \\
k
\end{array}\right)+\sum_{i=1}^{3} \frac{n_{i}-d-2+k}{k+1}\left(\begin{array}{c}
n_{i}-d-3+k \\
k
\end{array}\right) \\
& =\left(\begin{array}{c}
n_{[3]}-d-2+k \\
k+1
\end{array}\right)-\sum_{i=1}^{3}\left(\begin{array}{c}
n_{[3] \backslash\{i\}}-d-2+k \\
k+1
\end{array}\right)+\sum_{i=1}^{3}\left(\begin{array}{c}
n_{i}-d-2+k \\
k+1
\end{array}\right) \\
& =\sum_{\emptyset \subset S \subseteq[3]}(-1)^{3-|S|}\left(\begin{array}{c}
n_{S}-d-2+k \\
k+1
\end{array}\right) \text {, }
\end{aligned}
$$

where we used the fact that:

$$
\begin{aligned}
\sum_{i=1}^{3} \frac{n_{[3] \backslash\{i\}}}{k+1}\left(\begin{array}{c}
n_{i}-d-3+k \\
k
\end{array}\right) & =\sum_{i=1}^{3}\left(\sum_{j \in[3] \backslash\{i\}} \frac{n_{j}}{k+1}\right)\left(\begin{array}{c}
n_{i}-d-3+k \\
k
\end{array}\right)=\sum_{i=1}^{3} \sum_{j \in[3] \backslash\{i\}} \frac{n_{j}}{k+1}\left(\begin{array}{c}
n_{i}-d-3+k \\
k
\end{array}\right) \\
& =\sum_{i=1}^{3} \sum_{j \in[3] \backslash\{i\}} \frac{n_{i}}{k+1}\left(\begin{array}{c}
n_{j}-d-3+k \\
k
\end{array}\right)=\sum_{i=1}^{3} \frac{n_{i}}{k+1} \sum_{j \in[3] \backslash\{i\}}\left(\begin{array}{c}
n_{j}-d-3+k \\
k
\end{array}\right) .
\end{aligned}
$$

The rest of the proof is concerned with the equality claim. Assume first that $f_{l-1}\left(\mathcal{F}_{[3]}\right)=\sum_{\emptyset \subset S \subseteq[3]}(-1)^{3-|S|}\left(\begin{array}{c}n_{S} \\ l\end{array}\right)$, for all $0 \leq l \leq k$. Then we have:

$$
\begin{aligned}
h_{k}\left(\mathcal{F}_{[3]}\right) & =\sum_{i=0}^{d+2}(-1)^{k-i}\left(\begin{array}{c}
d+2-i \\
d+2-k
\end{array}\right) f_{i-1}\left(\mathcal{F}_{[3]}\right)=(-1)^{k} \sum_{i=0}^{d+2}(-1)^{i}\left(\begin{array}{c}
d+2-i \\
d+2-k
\end{array}\right) \sum_{\emptyset \subset S \subseteq[3]}(-1)^{3-|S|}\left(\begin{array}{c}
n_{S} \\
i
\end{array}\right) \\
& =(-1)^{k} \sum_{\emptyset \subset S \subseteq[3]}(-1)^{3-|S|} \sum_{i=0}^{d+2}(-1)^{i}\left(\begin{array}{c}
d+2-i \\
d+2-k
\end{array}\right)\left(\begin{array}{c}
n_{S} \\
i
\end{array}\right)=\sum_{\emptyset \subset S \subseteq[3]}(-1)^{3-|S|}\left(\begin{array}{c}
n_{S}-d-3+k \\
k
\end{array}\right) .
\end{aligned}
$$

In the above relation we used the combinatorial identity (cf. [13, eq. (5.25)]):

$$
\sum_{0 \leq k \leq l}\left(\begin{array}{c}
l-k \\
m
\end{array}\right)\left(\begin{array}{c}
s \\
k-n
\end{array}\right)(-1)^{k}=(-1)^{l+m}\left(\begin{array}{c}
s-m-1 \\
l-m-n
\end{array}\right)
$$


where $k \leftarrow i, l \leftarrow d+2, m \leftarrow d+2-k, n \leftarrow 0$, and $s \leftarrow n_{S}$.

Suppose now that $h_{k}\left(\mathcal{F}_{[3]}\right)=\sum_{\emptyset \subset S \subseteq[3]}(-1)^{3-|S|}\left(\begin{array}{c}n_{S}-d-3+k \\ k\end{array}\right)$. Since relation (37) holds for all $k \geq 0$, we conclude that $h_{l}\left(\mathcal{F}_{[3]}\right)$ must be equal to its upper bound in (41), for all $0 \leq l<k$. To see this suppose that (41) is not tight for some $l$, with $0 \leq l<k$, and among all such $l$ choose the largest one. Then, relation (37) implies that $h_{l+1}\left(\mathcal{F}_{[3]}\right)$ cannot be equal to its upper bound from (41), which contradicts the maximality of $l$. Hence, if $h_{k}\left(\mathcal{F}_{[3]}\right)$ is equal to its upper bound in $(41)$, so is $h_{l}\left(\mathcal{F}_{[3]}\right)$ for all $0 \leq l<k$, which gives, for all $l$ with $0 \leq l \leq k$ :

$$
\begin{aligned}
f_{l-1}\left(\mathcal{F}_{[3]}\right) & =\sum_{i=0}^{d+2}\left(\begin{array}{c}
d+2-i \\
l-i
\end{array}\right) h_{i}\left(\mathcal{F}_{[3]}\right)=\sum_{i=0}^{d+2}\left(\begin{array}{c}
d+2-i \\
l-i
\end{array}\right) \sum_{\emptyset \subset S \subseteq[3]}(-1)^{3-|S|}\left(\begin{array}{c}
n_{S}-d-3+i \\
i
\end{array}\right) \\
& =\sum_{\emptyset \subset S \subseteq[3]}(-1)^{3-|S|} \sum_{i=0}^{d+2}\left(\begin{array}{c}
d+2-i \\
l-i
\end{array}\right)\left(\begin{array}{c}
n_{S}-d-3+i \\
i
\end{array}\right) \\
& =\sum_{\emptyset \subset S \subseteq[3]}(-1)^{3-|S|} \sum_{i=0}^{d+2}\left(\begin{array}{c}
d+2-i \\
d+2-l
\end{array}\right)\left(\begin{array}{c}
n_{S}-d-3+i \\
n_{S}-d-3
\end{array}\right) \\
& =\sum_{\emptyset \subset S \subseteq[3]}(-1)^{3-|S|}\left(\begin{array}{c}
n_{S} \\
n_{S}-l
\end{array}\right)=\sum_{\emptyset \subset S \subseteq[3]}(-1)^{3-|S|}\left(\begin{array}{c}
n_{S} \\
l
\end{array}\right)
\end{aligned}
$$

where, in order to get from (42) to (43), we used the combinatorial identity (cf. [13, eq. (5.26)]):

$$
\sum_{0 \leq k \leq l}\left(\begin{array}{c}
l-k \\
m
\end{array}\right)\left(\begin{array}{c}
q+k \\
n
\end{array}\right)=\left(\begin{array}{c}
l+q+1 \\
m+n+1
\end{array}\right),
$$

with $k \leftarrow i, l \leftarrow d+2, m \leftarrow d+2-l, q \leftarrow n_{S}-d-3$, and $n \leftarrow n_{S}-d-3$.

We are now going to bound the elements of the $h$-vector of $\mathcal{K}_{[3]}$. These bounds are used below, in combination with the Dehn-Sommerville-like equations (15) in Lemma 4, to obtain refined upper bounds for the values of $h_{k}\left(\mathcal{F}_{[3]}\right)$ when $k>\left\lfloor\frac{d+2}{2}\right\rfloor$. More precisely:

Lemma 11. For all $0 \leq k \leq d+2$, we have:

$$
h_{k}\left(\mathcal{K}_{[3]}\right) \leq\left(\begin{array}{c}
n_{[3]}-d-3+k \\
k
\end{array}\right)
$$

Furthermore, for $d \geq 3$ and $d$ odd, we have:

$$
h_{\left\lfloor\frac{d}{2}\right\rfloor+1}\left(\mathcal{K}_{[3]}\right) \leq\left(\begin{array}{c}
n_{[3]}-\left\lfloor\frac{d}{2}\right\rfloor-3 \\
\left\lfloor\frac{d}{2}\right\rfloor+1
\end{array}\right)-\sum_{i=1}^{3}\left(\begin{array}{c}
n_{i}-\left\lfloor\frac{d}{2}\right\rfloor-2 \\
\left\lfloor\frac{d}{2}\right\rfloor+1
\end{array}\right) .
$$

Equality holds for some $k$, where $0 \leq k \leq\left\lfloor\frac{d+1}{2}\right\rfloor$, if and only if, for all $\emptyset \subset R \subseteq[3]$, $f_{l-1}\left(\mathcal{F}_{R}\right)=\sum_{\emptyset \subset S \subseteq R}(-1)^{|R|-|S|}\left(\begin{array}{c}n_{S} \\ l\end{array}\right)$, for all $0 \leq l \leq \min \left\{k,\left\lfloor\frac{d+|R|-1}{2}\right\rfloor\right\}$. 
Sketch of proof. The complete proof can be found in Section A.3 of Appendix A. To prove the upper bound for $h_{k}\left(\mathcal{K}_{[3]}\right)$, we distinguish between two cases: (1) the case $k=0$, where the result follows by a straightforward calculation from relation (13) with $R=[3]$, and (2) the case $k \geq 1$, where again we use (13) with $R=[3]$ and substitute $g_{k}\left(\mathcal{F}_{R}\right)$ by its upper bound from relation (40) in Lemma 9. We, thus, obtain a bound for $h_{k}\left(\mathcal{K}_{[3]}\right)$ expressed in terms of $h_{k}\left(\mathcal{F}_{[3]}\right), h_{k-1}\left(\mathcal{F}_{R}\right), R \in X_{2}$, and $g_{\lambda}\left(\partial P_{i}\right), \lambda=k, k-1$. Combining the upper bounds from Lemma 10, Lemma 3.3 in [19], along with the upper bounds for the $g$-vector of a $d$-polytope (cf. [31, Corollary 8.38]), respectively, gives the upper bound in the statement of the lemma.

For the equality claim we assume that $h_{k}\left(\mathcal{K}_{[3]}\right)$ attains its maximal value. Then, the expression bounding $h_{k}\left(\mathcal{K}_{[3]}\right)$ used above, in conjunction with Lemmas 8, 9, 10, and [19, Lemma 3.3], yields the equality conditions in the statement of the lemma. In the opposite direction, we assume that these conditions hold and, using Lemma 10 and [19, Lemma 3.3], we show that the quantities in the right hand side of relation (13) with $R=$ [3], attain their maximal values. The conclusion then follows from an easy calculation.

Utilizing the bounds from Lemmas 10 and 11, along with the Dehn-Sommerville-like equations (15), we arrive at the following theorem concerning upper bounds on the number of $k$-faces of the Minkowski sum of three convex $d$-polytopes in $\mathbb{R}^{d}$, as well as sufficient and necessary conditions for these bounds to be attained for all values of $k$.

Theorem 12. Let $P_{1}, P_{2}$ and $P_{3}$ be three d-polytopes in $\mathbb{R}^{d}, d \geq 2$, with $n_{i} \geq d+1$ vertices, $1 \leq i \leq 3$. Then, for all $1 \leq k \leq d$, we have:

$$
\begin{aligned}
f_{k-1}\left(P_{1}+P_{2}+P_{3}\right) \leq & f_{k+1}\left(C_{d+2}\left(n_{[3]}\right)\right)-\sum_{i=0}^{\left\lfloor\frac{d+2}{2}\right\rfloor}\left(\begin{array}{l}
d+2-i \\
k+2-i
\end{array}\right) \sum_{\emptyset \subset S \subset[3]}(-1)^{|S|}\left(\begin{array}{c}
n_{S}-d-3+i \\
i
\end{array}\right) \\
& -\delta\left(\begin{array}{c}
\left\lfloor\frac{d}{2}\right\rfloor+1 \\
k-\left\lfloor\frac{d}{2}\right\rfloor
\end{array}\right) \sum_{i=1}^{3}\left(\begin{array}{c}
n_{i}-\left\lfloor\frac{d}{2}\right\rfloor-2 \\
\left\lfloor\frac{d}{2}\right\rfloor+1
\end{array}\right),
\end{aligned}
$$

where $\delta=d-2\left\lfloor\frac{d}{2}\right\rfloor$, and $n_{S}=\sum_{i \in S} n_{i}$. Equality holds for all $1 \leq k \leq d$, if and only if

$$
f_{l-1}\left(\mathcal{F}_{R}\right)=\sum_{\emptyset \subset S \subseteq R}(-1)^{|R|-|S|}\left(\begin{array}{c}
n_{S} \\
l
\end{array}\right), \quad 0 \leq l \leq\left\lfloor\frac{d+|R|-1}{2}\right\rfloor, \quad \emptyset \subset R \subseteq[3] .
$$

Proof. If suffices to establish upper bounds for $f_{k}\left(\mathcal{F}_{[3]}\right)$ for all $0 \leq k \leq d+1$. Indeed, writing the $f$-vector of $\mathcal{F}_{[3]}$ in terms of its $h$-vector, and using relation (15), along with Lemmas 10 and 11 we get:

$$
\begin{aligned}
f_{k-1}\left(\mathcal{F}_{[3]}\right) & =\sum_{i=0}^{d+2}\left(\begin{array}{c}
d+2-i \\
k-i
\end{array}\right) h_{i}\left(\mathcal{F}_{[3]}\right)=\sum_{i=0}^{\left\lfloor\frac{d+2}{2}\right\rfloor}\left(\begin{array}{c}
d+2-i \\
k-i
\end{array}\right) h_{i}\left(\mathcal{F}_{[3]}\right)+\sum_{i=\left\lfloor\frac{d+2}{2}\right\rfloor+1}^{d+2}\left(\begin{array}{c}
d+2-i \\
k-i
\end{array}\right) h_{i}\left(\mathcal{F}_{[3]}\right) \\
& =\sum_{i=0}^{\left\lfloor\frac{d+2}{2}\right\rfloor}\left(\begin{array}{c}
d+2-i \\
k-i
\end{array}\right) h_{i}\left(\mathcal{F}_{[3]}\right)+\sum_{j=0}^{\left\lfloor\frac{d+1}{2}\right\rfloor}\left(\begin{array}{c}
j \\
k-d-2+j
\end{array}\right) h_{d+2-j}\left(\mathcal{F}_{[3]}\right)
\end{aligned}
$$




$$
=\sum_{i=0}^{\left\lfloor\frac{d+2}{2}\right\rfloor}\left(\begin{array}{c}
d+2-i \\
k-i
\end{array}\right) h_{i}\left(\mathcal{F}_{[3]}\right)+\sum_{j=0}^{\left\lfloor\frac{d+1}{2}\right\rfloor}\left(\begin{array}{c}
j \\
k-d-2+j
\end{array}\right) h_{j}\left(\mathcal{K}_{[3]}\right) .
$$

From Lemma 10 we have:

$$
\sum_{i=0}^{\left\lfloor\frac{d+2}{2}\right\rfloor}\left(\begin{array}{c}
d+2-i \\
k-i
\end{array}\right) h_{i}\left(\mathcal{F}_{[3]}\right) \leq \sum_{i=0}^{\left\lfloor\frac{d+2}{2}\right\rfloor}\left(\begin{array}{c}
d+2-i \\
k-i
\end{array}\right) \sum_{\emptyset \subset S \subseteq[3]}(-1)^{3-|S|}\left(\begin{array}{c}
n_{S}-d-3+i \\
i
\end{array}\right),
$$

whereas from Lemma 11 we get

$$
\sum_{j=0}^{\left\lfloor\frac{d+1}{2}\right\rfloor}\left(\begin{array}{c}
j \\
k-d-2+j
\end{array}\right) h_{j}\left(\mathcal{K}_{[3]}\right) \leq \sum_{i=0}^{\left\lfloor\frac{d+1}{2}\right\rfloor}\left(\begin{array}{c}
j \\
k-d-2+j
\end{array}\right)\left(\begin{array}{c}
n_{[3]}-d-3+j \\
j
\end{array}\right)-\delta\left(\begin{array}{c}
\left\lfloor\frac{d}{2}\right\rfloor+1 \\
k-\left\lfloor\frac{d}{2}\right\rfloor-2
\end{array}\right) \sum_{i=1}^{3}\left(\begin{array}{c}
n_{i}-\left\lfloor\frac{d}{2}\right\rfloor-2 \\
\left\lfloor\frac{d}{2}\right\rfloor+1
\end{array}\right),
$$

where $\delta=d-2\left\lfloor\frac{d}{2}\right\rfloor$. Hence:

$$
\begin{aligned}
& f_{k-1}\left(\mathcal{F}_{[3]}\right) \leq \sum_{i=0}^{\left\lfloor\frac{d+2}{2}\right\rfloor}\left(\begin{array}{c}
d+2-i \\
k-i
\end{array}\right) \sum_{\emptyset \subset S \subseteq[3]}(-1)^{3-|S|}\left(\begin{array}{c}
n_{S}-d-3+i \\
i
\end{array}\right)+\sum_{j=0}^{\left\lfloor\frac{d+1}{2}\right\rfloor}\left(\begin{array}{c}
j \\
k-d-2+j
\end{array}\right)\left(\begin{array}{l}
n_{[3]}-d-3+j \\
j
\end{array}\right) \\
& -\delta\left(\begin{array}{c}
\left\lfloor\frac{d}{2}\right\rfloor+1 \\
k-\left\lfloor\frac{d}{2}\right\rfloor-2
\end{array}\right) \sum_{i=1}^{3}\left(\begin{array}{c}
n_{i}-\left\lfloor\frac{d}{2}\right\rfloor-2 \\
\left\lfloor\frac{d}{2}\right\rfloor+1
\end{array}\right) \\
& =\sum_{i=0}^{\left\lfloor\frac{d+2}{2}\right\rfloor}\left(\begin{array}{c}
d+2-i \\
k-i
\end{array}\right)\left(\begin{array}{c}
n_{[3]}-d-3+i \\
i
\end{array}\right)+\sum_{i=0}^{\left\lfloor\frac{d+1}{2}\right\rfloor}\left(\begin{array}{c}
i \\
k-d-2+i
\end{array}\right)\left(\begin{array}{c}
n_{[3]}-d-3+i \\
i
\end{array}\right) \\
& -\sum_{i=0}^{\left\lfloor\frac{d+2}{2}\right\rfloor}\left(\begin{array}{c}
d+2-i \\
k-i
\end{array}\right) \sum_{\emptyset \subset S \subset[3]}(-1)^{|S|}\left(\begin{array}{c}
n_{S}-d-3+i \\
i
\end{array}\right)-\delta\left(\begin{array}{c}
\left\lfloor\frac{d}{2}\right\rfloor+1 \\
k-\left\lfloor\frac{d}{2}\right\rfloor-2
\end{array}\right) \sum_{i=1}^{3}\left(\begin{array}{c}
n_{i}-\left\lfloor\frac{d}{2}\right\rfloor-2 \\
\left\lfloor\frac{d}{2}\right\rfloor+1
\end{array}\right) \\
& =\sum_{i=0}^{\frac{d+2}{2}} *\left(\left(\begin{array}{c}
d+2-i \\
k-i
\end{array}\right)+\left(\begin{array}{c}
i \\
k-d-2+i
\end{array}\right)\right)\left(\begin{array}{c}
n_{[3]}-d-3+i \\
i
\end{array}\right) \\
& -\sum_{i=0}^{\left\lfloor\frac{d+2}{2}\right\rfloor}\left(\begin{array}{c}
d+2-i \\
k-i
\end{array}\right) \sum_{\emptyset \subset S \subset[3]}(-1)^{|S|}\left(\begin{array}{c}
n_{S}-d-3+i \\
i
\end{array}\right)-\delta\left(\begin{array}{c}
\left\lfloor\frac{d}{2}\right\rfloor+1 \\
k-\left\lfloor\frac{d}{2}\right\rfloor-2
\end{array}\right) \sum_{i=1}^{3}\left(\begin{array}{c}
n_{i}-\left\lfloor\frac{d}{2}\right\rfloor-2 \\
\left\lfloor\frac{d}{2}\right\rfloor+1
\end{array}\right) \\
& =f_{k-1}\left(C_{d+2}\left(n_{[3]}\right)\right)-\sum_{i=0}^{\left\lfloor\frac{d+2}{2}\right\rfloor}\left(\begin{array}{c}
d+2-i \\
k-i
\end{array}\right) \sum_{\emptyset \subset S \subset[3]}(-1)^{|S|}\left(\begin{array}{c}
n_{S}-d-3+i \\
i
\end{array}\right) \\
& -\delta\left(\begin{array}{c}
\left\lfloor\frac{d}{2}\right\rfloor+1 \\
k-\left\lfloor\frac{d}{2}\right\rfloor-2
\end{array}\right) \sum_{i=1}^{3}\left(\begin{array}{c}
n_{i}-\left\lfloor\frac{d}{2}\right\rfloor-2 \\
\left\lfloor\frac{d}{2}\right\rfloor+1
\end{array}\right),
\end{aligned}
$$

where:

$$
\sum_{i=0}^{\frac{m}{2}}{ }^{*} T_{i}=\sum_{i=0}^{\left\lfloor\frac{m}{2}\right\rfloor-1} T_{i}+\frac{1}{2}\left(1+m-2\left\lfloor\frac{m}{2}\right\rfloor\right) T_{\left\lfloor\frac{m}{2}\right\rfloor}
$$


Our upper bounds follow from the fact that $f_{k-1}\left(P_{1}+P_{2}+P_{3}\right)=f_{k+1}\left(\mathcal{F}_{[3]}\right), 1 \leq k \leq d$.

In what follows we concentrate on the necessary and sufficient conditions for the upper bounds in (46) to hold as equalities. From the derivation of the upper bounds above (see also relation (48)), it is clear that the bounds are tight if and only if:

(i) $h_{k}\left(\mathcal{F}_{[3]}\right)$ is maximal, for all $0 \leq k \leq\left\lfloor\frac{d+2}{2}\right\rfloor$, and

(ii) $h_{k}\left(\mathcal{K}_{[3]}\right)$ is maximal, for all $0 \leq k \leq\left\lfloor\frac{d+1}{2}\right\rfloor$.

According to Lemma 10 and Lemma 11, these conditions are, respectively, equivalent to requiring that:

(a) $f_{l-1}\left(\mathcal{F}_{[3]}\right)=\sum_{\emptyset \subset S \subseteq[3]}(-1)^{3-|S|}\left(\begin{array}{c}n_{S} \\ l\end{array}\right)$, for all $0 \leq l \leq\left\lfloor\frac{d+2}{2}\right\rfloor$, and

(b) $f_{l-1}\left(\mathcal{F}_{R}\right)=\sum_{\emptyset \subset S \subseteq R}(-1)^{|R|-|S|}\left(\begin{array}{c}n_{S} \\ l\end{array}\right)$, for all $0 \leq l \leq \min \left\{\left\lfloor\frac{d+1}{2}\right\rfloor,\left\lfloor\frac{d+|R|-1}{2}\right\rfloor\right\}$, and for all $\emptyset \subset R \subseteq[3]$.

For $R \equiv[3]$, condition (a) implies condition (b), while for $R \subset[3], \min \left\{\left\lfloor\frac{d+1}{2}\right\rfloor,\left\lfloor\frac{d+|R|-1}{2}\right\rfloor\right\}=$ $\left\lfloor\frac{d+|R|-1}{2}\right\rfloor$. We, therefore, conclude that the bounds in (46) are attained if and only if, conditions (47) hold true for all $0 \leq k \leq\left\lfloor\frac{d-|R|+1}{2}\right\rfloor$ and for all $\emptyset \subset R \subseteq[3]$.

\section{Tightness of upper bounds}

In this section we show that the bounds in Theorem 12 are tight. We distinguish between the cases $d=2, d=3$ and $d \geq 4$. For $d=2$, it is easy to verify that for $k=1,2$, the right-hand side of inequality (46) evaluates to $n_{1}+n_{2}+n_{3}$, which is known to be tight.

\subsection{Three dimensions}

For $d=3$, the upper bounds in Theorem 12 are as follows:

$$
\begin{aligned}
& f_{0}\left(P_{1}+P_{2}+P_{3}\right) \leq n_{1} n_{2}+n_{2} n_{3}+n_{1} n_{3}-n_{1}-n_{2}-n_{3}+2, \\
& f_{1}\left(P_{1}+P_{2}+P_{3}\right) \leq 2 n_{1} n_{2}+2 n_{2} n_{3}+2 n_{1} n_{3}-n_{1}-n_{2}-n_{3}-6, \\
& f_{2}\left(P_{1}+P_{2}+P_{3}\right) \leq n_{1} n_{2}+n_{2} n_{3}+n_{1} n_{3}-6 .
\end{aligned}
$$

In order to prove that these bounds are tight, we exploit two results: one by Fukuda and Weibel [10] and one by Weibel [30]. Weibel [30] has shown that the number of $k$-faces of the Minkowski sum of $r d$-polytopes $P_{1}, \ldots, P_{r}$ in $\mathbb{R}^{d}$, where $r \geq d$, is related to the number of $k$-faces of the Minkowski sum of subsets of these polytopes of size at most $d-1$ as follows:

$$
f_{k}\left(P_{1}+P_{2}+\cdots+P_{r}\right)-\alpha=\sum_{j=1}^{d-1}(-1)^{d-1-j}\left(\begin{array}{c}
r-1-j \\
d-1-j
\end{array}\right) \sum_{S \in X_{j}^{r}}\left(f_{k}\left(P_{S}\right)-\alpha\right),
$$


where $X_{j}^{r}$ is the family of subsets of $[r]$ of size $j, P_{S}$ is the Minkowski sum of the polytopes in $S$, and $\alpha=2$ if $k=0$ and $d$ is odd, and $\alpha=0$ otherwise. For $d=r=3$, equation (50) simplifies to:

$$
\begin{aligned}
f_{k}\left(P_{1}+P_{2}+P_{3}\right) & =\alpha+\sum_{j=1}^{2}(-1)^{2-j}\left(\begin{array}{l}
2-j \\
2-j
\end{array}\right) \sum_{S \in X_{j}^{3}}\left(f_{k}\left(P_{S}\right)-\alpha\right) \\
& =\alpha-\sum_{i=1}^{3}\left(f_{k}\left(P_{i}\right)-\alpha\right)+\sum_{i=1}^{3}\left(f_{k}\left(P_{[3] \backslash\{i\}}\right)-\alpha\right) \\
& =\alpha-\sum_{i=1}^{3} f_{k}\left(P_{i}\right)+3 \alpha+\sum_{i=1}^{3} f_{k}\left(P_{[3] \backslash\{i\}}\right)-3 \alpha \\
& =\alpha+\sum_{1 \leq i<j \leq 3} f_{k}\left(P_{i}+P_{j}\right)-\sum_{i=1}^{3} f_{k}\left(P_{i}\right) .
\end{aligned}
$$

Besides relation (50), Weibel [30] also presented a construction of $r$ simplicial $d$ polytopes, such that any subset $S$ of these polytopes of size at most $d-1$ has the maximum possible number of vertices, namely, $f_{0}\left(P_{S}\right)=\prod_{i \in S} n_{i}$. Specializing this construction in our case, i.e., for $r=d=3$, we deduce that it is possible to construct three simplicial 3-polytopes $P_{1}, P_{2}, P_{3}$ in $\mathbb{R}^{3}$, such that $f_{0}\left(P_{i}\right)=n_{i}, 1 \leq i \leq 3$, and $f_{0}\left(P_{i}+P_{j}\right)=n_{i} n_{j}$, $1 \leq i<j \leq 3$. Substituting in (51) for $k=0$, we get:

$$
f_{0}\left(P_{1}+P_{2}+P_{3}\right)=2+\sum_{1 \leq i<j \leq 3} n_{i} n_{j}-\sum_{i=1}^{3} n_{i}=n_{1} n_{2}+n_{2} n_{3}+n_{1} n_{3}-n_{1}-n_{2}-n_{3}+2
$$

i.e., the upper bound in (49) is tight for $k=0 .{ }^{4}$ Since all $P_{i}$ 's are simplicial, we have

$$
f_{1}\left(P_{i}\right)=3 n_{i}-6, \quad f_{2}\left(P_{i}\right)=2 n_{i}-4, \quad 1 \leq i \leq 3 .
$$

On the other hand, since $f_{0}\left(P_{i}+P_{j}\right)$ is maximal, for all $1 \leq i<j \leq 3$, we get, by [10, Corollary 4], that $f_{k}\left(P_{i}+P_{j}\right)$ is also maximal for $k=1,2$, and for all $1 \leq i<j \leq 3$. Hence:

$$
f_{1}\left(P_{i}+P_{j}\right)=2 n_{i} n_{j}+n_{i}+n_{j}-8, \quad f_{2}\left(P_{i}+P_{j}\right)=n_{i} n_{j}+n_{i}+n_{j}-6 .
$$

Substituting from (52) and (53) in (51), and recalling that $\alpha=0$ for $k>0$, we get:

$$
\begin{aligned}
f_{1}\left(P_{1}+P_{2}+P_{3}\right)= & \sum_{1 \leq i<j \leq 3}\left(2 n_{i} n_{j}+n_{i}+n_{j}-8\right)-\sum_{i=1}^{3}\left(3 n_{i}-6\right) \\
= & {\left[2\left(n_{1} n_{2}+n_{2} n_{3}+n_{1} n_{3}\right)+2\left(n_{1}+n_{2}+n_{3}\right)-24\right] } \\
& \quad-\left[3\left(n_{1}+n_{2}+n_{3}\right)-18\right] \\
= & 2 n_{1} n_{2}+2 n_{2} n_{3}+2 n_{1} n_{3}-n_{1}-n_{2}-n_{3}-6,
\end{aligned}
$$

\footnotetext{
${ }^{4}$ This is essentially the result of Theorem 3 in [30] for $d=r=3$; however, we recapitulate this result in order to show that Weibel's construction yields tights bounds for $k=1,2$ also.
} 
and

$$
\begin{aligned}
f_{2}\left(P_{1}+P_{2}+P_{3}\right) & =\sum_{1 \leq i<j \leq 3}\left(n_{i} n_{j}+n_{i}+n_{j}-6\right)-\sum_{i=1}^{3}\left(2 n_{i}-4\right) \\
& =\left[n_{1} n_{2}+n_{2} n_{3}+n_{1} n_{3}+2\left(n_{1}+n_{2}+n_{3}\right)-18\right]-\left[2\left(n_{1}+n_{2}+n_{3}\right)-12\right] \\
& =n_{1} n_{2}+n_{2} n_{3}+n_{1} n_{3}-6,
\end{aligned}
$$

i.e., the upper bounds in (49) are tight for $k=1,2$.

\subsection{Four or more dimensions}

We now focus on the case $d \geq 4$. We shall construct three $d$-polytopes $P_{1}, P_{2}$ and $P_{3}$ in $\mathbb{R}^{d}$, such that they satisfy the conditions in relation (47). Consequently, as Theorem 12 asserts, these polytopes attain the upper bounds in (46).

Consider the following $d$-dimensional moment-like curves in $\mathbb{R}^{d}$ :

$$
\begin{aligned}
& \gamma_{1}(t)=\left(t, \zeta t^{2}, \zeta t^{3}, t^{4}, t^{5}, \ldots, t^{d}\right), \\
& \gamma_{2}(t)=\left(\zeta t, t^{2}, \zeta t^{3}, t^{4}, t^{5}, \ldots, t^{d}\right), \\
& \gamma_{3}(t)=\left(\zeta t, \zeta t^{2}, t^{3}, t^{4}, t^{5}, \ldots, t^{d}\right),
\end{aligned}
$$

where $t>0$, and $\zeta \geq 0$. Let $\boldsymbol{e}_{1,1}=(0), \boldsymbol{e}_{1,2}=(1)$ be the standard affine basis of $\mathbb{R}$ and recall that $\boldsymbol{e}_{2,1}=(0,0), \boldsymbol{e}_{2,2}=(1,0), \boldsymbol{e}_{2,3}=(0,1)$ is the standard affine basis of $\mathbb{R}^{2}$. We shall define three neighborly polytopes as the convex hulls of points, chosen appropriately on each of these $d$-curves. We then proceed to show that $\mathcal{F}_{R}, R \in X_{2}$, and $\mathcal{F}_{[3]}$, have the following property: every set of $k=\left\lfloor\frac{d+1}{2}\right\rfloor$ vertices from $\mathcal{F}_{R}$, or $k \leq\left\lfloor\frac{d+2}{2}\right\rfloor$ vertices from $\mathcal{F}_{[3]}$, defines a $(k-1)$-face of $\mathcal{F}_{R}$ or $\mathcal{F}_{[3]}$, respectively. This property readily yields the necessary and sufficient conditions establishing the tightness of the upper bounds (cf. rel. (47)).

Let $x_{i, j}, 1 \leq j \leq n_{i}, 1 \leq i \leq 3$, be $n_{[3]}$ positive real numbers, such that $x_{i, j}<x_{i, j+1}$, $1 \leq j \leq n_{i}-1$, and let $\tau$ be a positive real parameter. Let $x_{i, j}^{\epsilon}=x_{i, j}+\epsilon, t_{i, j}=x_{i, j} \tau^{\nu_{i}}$, $t_{i, j}^{\epsilon}=x_{i, j}^{\epsilon} \tau^{\nu_{i}}$, where $1 \leq j \leq n_{i}, 1 \leq i \leq 3, \epsilon>0$, and $\nu_{i}=3-i, 1 \leq i \leq 3$. The value of $\epsilon$ is chosen such that $x_{i, j}^{\epsilon}<x_{i, j+1}$, for all $1 \leq j<n_{i}$, and for all $1 \leq i \leq 3$. Finally, we set $\zeta=\tau^{M}$, where $M \geq d(d+1)$. We are going to define three vertex sets $V_{i}$ as follows:

$$
V_{i}=\left\{\gamma_{i}\left(t_{i, 1}\right), \gamma_{i}\left(t_{i, 2}\right), \ldots \gamma_{i}\left(t_{i, n_{i}}\right)\right\} \quad 1 \leq i \leq 3
$$

Call $P_{i}$ the $d$-polytope we get as the convex hull of the vertices in $V_{i}$, and let $\mathcal{V}_{i}$ be the image of $V_{i}$ via the Cayley embedding. As in Section 2, call $\mathcal{C}$ the Cayley polytope of the $P_{i}$ 's in $\mathbb{R}^{d+2}$, and $\mathcal{F}_{R}, \emptyset \subset R \subseteq[3]$, the set of faces of $\mathcal{C}$ with at least one vertex from each $\mathcal{V}_{i}$, $i \in R$, and no vertex from any $\mathcal{V}_{j}, j \notin R$. Note that, by construction, $P_{i}$ is a $\left\lfloor\frac{d}{2}\right\rfloor$-neighborly polytope in $\mathbb{R}^{d}$ with $n_{i}$ vertices, which immediately implies that conditions (47) hold for $R \in X_{1}$ and for all $0 \leq l \leq\left\lfloor\frac{d}{2}\right\rfloor$. Hence, it suffices to show that:

$$
f_{l-1}\left(\mathcal{F}_{R}\right)=\sum_{\emptyset \subset S \subseteq R}(-1)^{|R|-|S|}\left(\begin{array}{c}
n_{S} \\
l
\end{array}\right), \quad 0 \leq l \leq\left\lfloor\frac{d+|R|-1}{2}\right\rfloor, \quad 2 \leq|R| \leq 3,
$$


which we will succeed by choosing a sufficiently small value for $\tau$.

To prove that the constructed polytopes have the desired properties (see Lemmas 13 and 14, bellow), we adopt the key idea used in the proofs of [31, Theorem $0.7 \&$ Corollary 0.8 ] on basic properties of cyclic $d$-polytopes, and adapt this idea to our setting, where we view the faces of the Minkowski sum of the polytopes $P_{i}, i \in R$, via the face set $\mathcal{F}_{R}$ of their Cayley polytope, where $2 \leq|R| \leq 3$.

We start off with subsets $R$ of size two. To show that $f_{k-1}\left(\mathcal{F}_{R}\right)$ is according to relation (55), recall (cf. Section 2) that the polytope $\mathcal{C}$ contains the Cayley polytope $\mathcal{C}_{R}$ of the polytopes in $R$ as a facet. Thus, in order to prove relation (55) for $\mathcal{F}_{R}$, we may consider $\mathcal{C}_{R}$ and $\mathcal{F}_{R}$ independently of $\mathcal{C}$, i.e., we can disassociate the polytopes $P_{i}, i \in R$, from the Cayley polytope $\mathcal{C}$. In other words, we think of the polytopes $P_{i}, i \in R$, as $d$-polytopes in $\mathbb{R}^{d}$, while their Cayley polytope $\mathcal{C}_{R}$ is seen as a $(d+1)$-polytope in $\mathbb{R}^{d+1}$. We exploit this observation in order to prove the following lemma.

Lemma 13. There exists a sufficiently small positive value $\hat{\tau}_{R}$ for $\tau$ such that, for all $\tau \in\left(0, \hat{\tau}_{R}\right)$,

$$
f_{k-1}\left(\mathcal{F}_{R}\right)=\sum_{\emptyset \subset S \subseteq R}(-1)^{2-|S|}\left(\begin{array}{c}
n_{S} \\
k
\end{array}\right), \quad 2 \leq k \leq\left\lfloor\frac{d+1}{2}\right\rfloor, \quad R \in X_{2} .
$$

Proof. Without loss of generality let $R=\{1,3\}$. The rest of the cases are analogous. The condition in the statement of the lemma is equivalent to the requirement that $\mathcal{C}_{\{1,3\}}$ is a $\left(\mathcal{V}_{1},\left\lfloor\frac{d+1}{2}\right\rfloor\right)$-bineighborly polytope (see [19] for definitions and details), which in turn is equivalent to the requirement that

$$
f_{\left\lfloor\frac{d+1}{2}\right\rfloor-1}\left(\mathcal{F}_{\{1,3\}}\right)=\sum_{\emptyset \subset S \subseteq\{1,3\}}(-1)^{2-|S|}\left(\begin{array}{c}
n_{S} \\
\left\lfloor\frac{d+1}{2}\right\rfloor
\end{array}\right) .
$$

We shall prove that condition (56) holds true for the Cayley polytope $\mathcal{C}_{\{1,3\}}$ of the polytopes $P_{1}, P_{3}$, and for sufficiently small values of $\tau$, as described in the statement of the lemma.

Define $\delta:=d+1-2\left\lfloor\frac{d+1}{2}\right\rfloor$. Let $X$ be a positive real number such that $X>x_{3, n_{3}}^{\epsilon}$, and let $^{5} T=X \tau^{\nu_{3}}$. Choose a set $U$ of $k_{m} \neq 0$ vertices $\gamma_{m}\left(t_{m, j_{m, 1}}\right), \gamma_{m}\left(t_{m, j_{m, 2}}\right), \ldots, \gamma_{m}\left(t_{m, j_{m, k_{m}}}\right)$ from the set $V_{m}$, such that $j_{m, 1}<j_{m, 2}<\ldots<j_{m, k_{m}}$, for $m \in\{1,3\}$, and $k_{1}+k_{3}=\left\lfloor\frac{d+1}{2}\right\rfloor$. Let $\mathcal{U}=\left\{\boldsymbol{\beta}_{m}\left(t_{m, j_{m, 1}}\right), \boldsymbol{\beta}_{m}\left(t_{m, j_{m, 2}}\right), \ldots, \boldsymbol{\beta}_{m}\left(t_{m, j_{m, k}}\right) \mid m \in\{1,3\}\right\}$, be the Cayley embed$\operatorname{ding}$ of $U$ in $\mathbb{R}^{d+1}$ (using the affine basis $\left.\boldsymbol{e}_{1,1}, \boldsymbol{e}_{1,3}\right)$. For a vector $\boldsymbol{x}=\left(x_{1}, x_{2}, \ldots, x_{d+1}\right) \in$ $\mathbb{R}^{d+1}$, we define the $(d+2) \times(d+2)$ determinant $H_{\mathfrak{U}}(\boldsymbol{x})$ as follows:

\footnotetext{
${ }^{5}$ Although we have set $\nu_{3}=0$, we keep $\nu_{3}$ as is in the proof, so as to make more profound the analogy of the proof presented here for $R=\{1,3\}$ with the cases $R=\{1,2\}$ and $R=\{2,3\}$.
} 


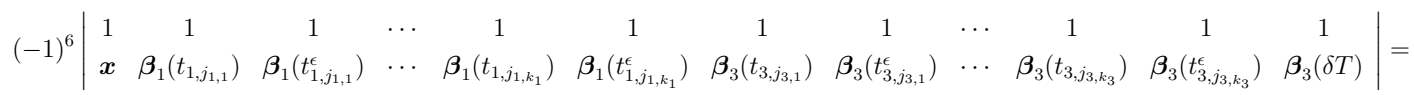

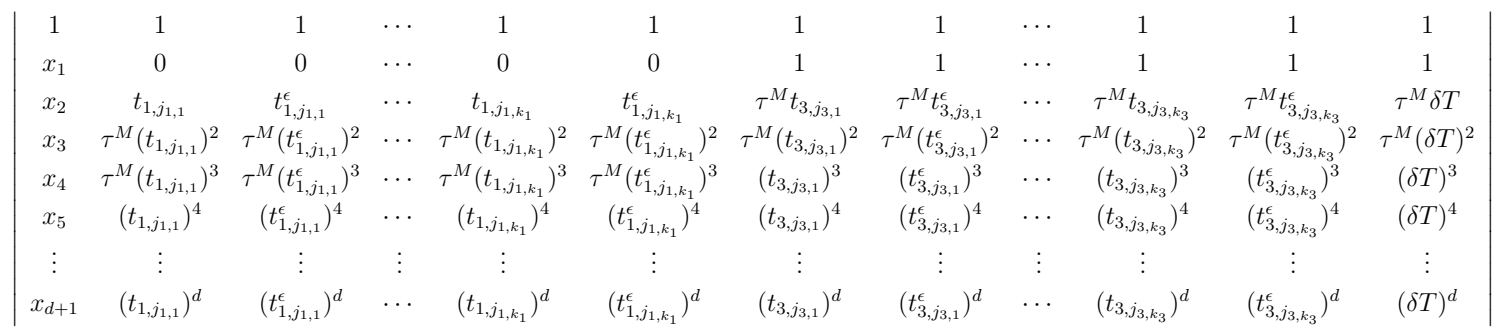

Notice that for $d$ odd the last column $\left(\begin{array}{c}1 \\ \boldsymbol{\beta}_{3}(\delta T)\end{array}\right)$ of $H_{\mathcal{U}}(x)$ does not exist. The equation $H_{\mathcal{U}}(\boldsymbol{x})=0$ is the equation of a hyperplane in $\mathbb{R}^{d+1}$ that passes through the points in $\mathcal{U}$. We are going to show that, for any choice of $\mathcal{U}$, and for all vertices $\boldsymbol{v}$ in $\mathcal{V}_{\{1,3\}} \backslash \mathcal{U}$, $\mathcal{V}_{\{1,3\}}=\mathcal{V}_{1} \cup \mathcal{V}_{3}$, we have $H_{\mathcal{U}}(\boldsymbol{v})>0$ for sufficiently small values of $\tau$.

Suppose we have some vertex $\boldsymbol{v} \in \mathcal{V}_{\{1,3\}} \backslash \mathcal{U}$. Then, $\boldsymbol{v}=\boldsymbol{\beta}_{s}\left(t_{s, \lambda}\right), t_{s, \lambda}=x_{s, \lambda} \tau^{\nu_{s}}$, where $1 \leq \lambda \leq n_{s}, s$ is either 1 or 3 , and $\lambda \notin\left\{j_{s, 1}, j_{s, 2}, \ldots, j_{s, k_{s}}\right\}$. We perform the following determinant transformations on $H_{\mathfrak{U}}(\boldsymbol{v})$ : initially we subtract its second row from its first, and then we shift its first column to the right via an even number of column swaps. More precisely, we need to shift the first column of $H_{U}(\boldsymbol{v})$ to the right so that the values $t_{s, \lambda}, t_{s, j_{s, 1}}, t_{s, j_{s, 1}}^{\epsilon}, t_{s, j_{s, 2}}, t_{s, j_{s, 2}}^{\epsilon}, \ldots, t_{s, j_{s, k_{s}}}, t_{s, j_{s, k_{s}}}^{\epsilon}$ appear consecutively in the columns of $H_{\mathfrak{U}}(\boldsymbol{v})$ and in increasing order. To do that we always need an even number of column swaps, due to the way we have chosen $\epsilon$.

Consider the case where $s=1$ and suppose that all necessary operations on $H_{\mathcal{U}}(\boldsymbol{v})$ have been performed. Then $H_{\mathcal{U}}(\boldsymbol{v})$ is in the form of the determinant $D_{n, m}(\tau ; I, J, \boldsymbol{\mu})$ of Lemma 16 (multiplied by $\tau^{M}$ ), with $n \leftarrow 2 k_{1}+1, m \leftarrow 2 k_{3}, l \leftarrow d+2, \boldsymbol{\mu} \leftarrow(0,0,1,2, \ldots, d$ ), $\alpha \leftarrow \nu_{1}, \beta \leftarrow \nu_{3}, I \leftarrow 3$, and $J \leftarrow 5$. Note that the requirement for $M$ in Lemma 16 is satisfied by our choice of $M$. According to Lemma 16 (see Section B of the Appendix), $H_{\mathcal{U}}(\boldsymbol{v})$ has the following asymptotic expansion in terms of $\tau$ :

$$
H_{\mathcal{U}}(\boldsymbol{v})=\tau^{M}\left(C \tau^{\xi}+\Theta\left(\tau^{\xi+1}\right)\right), \quad \xi=\nu_{1}\left(-2+\sum_{i=4}^{2 k_{1}+3}(i-2)\right)+\nu_{3}\left(3+\sum_{i=2 k_{1}+4}^{d+2}(i-2)\right),
$$

where $C$ is a positive constant independent of $\tau$. The asymptotic expansion in (57) implies that there exists a positive value $\hat{\tau}_{\boldsymbol{v}}, \mathfrak{U}$ for $\tau$ such that for all $\tau \in\left(0, \hat{\tau}_{\boldsymbol{v}}, u\right), H_{\mathcal{U}}(\boldsymbol{v})>0$. The case $s=3$ is completely analogous.

Since the number of the subsets $\mathcal{U}$ is finite, while for each such subset $\mathcal{U}$ we need to consider a finite number of vertices in $\mathcal{V}_{\{1,3\}} \backslash \mathcal{U}$, it suffices to consider a positive value $\hat{\tau}_{\{1,3\}}$ for $\tau$ that is small enough, so that all possible determinants $H_{\mathfrak{U}}(\boldsymbol{v})$ are strictly positive for any $\tau \in\left(0, \hat{\tau}_{\{1,3\}}\right)$. For $\tau \in\left(0, \hat{\tau}_{\{1,3\}}\right)$, our analysis above immediately implies that for each set $\mathcal{U}$ the equation $H_{\mathcal{U}}(\boldsymbol{x})=0, \boldsymbol{x} \in \mathbb{R}^{d+1}$, is the equation of a supporting hyperplane of $\mathcal{C}_{R}$ passing through the vertices of $\mathcal{U}$, and those only. In other words, every set $\mathcal{U}$, where $|\mathcal{U}|=\left\lfloor\frac{d+1}{2}\right\rfloor,\left|\mathcal{\cap} \cap \mathcal{V}_{1}\right|=k_{1} \neq 0$, and $\left|\mathcal{U} \cap \mathcal{V}_{3}\right|=k_{3} \neq 0$, defines a $\left(\left\lfloor\frac{d+1}{2}\right\rfloor-1\right)$-face of $\mathcal{C}_{R}$. Taking into account that the number of such subsets $\mathcal{U}$ is $\sum_{i=1}^{\left\lfloor\frac{d+1}{2}\right\rfloor-1}\left(\begin{array}{c}n_{1} \\ i\end{array}\right)\left(\begin{array}{c}n_{3} \\ \left\lfloor\frac{d+1}{2}\right\rfloor-i\end{array}\right)$, we deduce 
that

$$
\begin{aligned}
f_{\left\lfloor\frac{d+1}{2}\right\rfloor-1}\left(\mathcal{F}_{\{1,3\}}\right) & =\sum_{i=1}^{\left\lfloor\frac{d+1}{2}\right\rfloor-1}\left(\begin{array}{c}
n_{1} \\
i
\end{array}\right)\left(\begin{array}{c}
n_{3} \\
\left\lfloor\frac{d+1}{2}\right\rfloor-i
\end{array}\right)=\left(\begin{array}{c}
n_{1}+n_{3} \\
\left\lfloor\frac{d+1}{2}\right\rfloor
\end{array}\right)-\left(\begin{array}{c}
n_{1} \\
\left\lfloor\frac{d+1}{2}\right\rfloor
\end{array}\right)-\left(\begin{array}{c}
n_{3} \\
\left\lfloor\frac{d+1}{2}\right\rfloor
\end{array}\right) \\
& =\sum_{\emptyset \subset S \subseteq\{1,3\}}(-1)^{2-|S|}\left(\begin{array}{c}
n_{S} \\
\left\lfloor\frac{d+1}{2}\right\rfloor
\end{array}\right) .
\end{aligned}
$$

Hence, condition (56) is satisfied for all $\tau \in\left(0, \hat{\tau}_{\{1,3\}}\right)$.

We now consider the case $R=[3]$. In this case we can show that:

Lemma 14. There exists a sufficiently small positive value $\hat{\tau}_{[3]}$ for $\tau$ such that, for all $\tau \in\left(0, \hat{\tau}_{[3]}\right)$,

$$
f_{k-1}\left(\mathcal{F}_{[3]}\right)=\sum_{\emptyset \subset S \subseteq[3]}(-1)^{3-|S|}\left(\begin{array}{c}
n_{S} \\
k
\end{array}\right), \quad 3 \leq k \leq\left\lfloor\frac{d+2}{2}\right\rfloor
$$

Proof. Define $\delta:=d+2-2 k$ and let $T$ be a positive real number such that $T>t_{3, n_{3}}^{\epsilon}\left(=x_{3, n_{3}}^{\epsilon}\right)$. Choose a set $U$ of $k_{i} \neq 0$ vertices from $V_{i}, 1 \leq i \leq 3$, such that $k_{1}+k_{2}+k_{3}=k$, and denote by $\mathcal{U}$ the Cayley embedding of $U$ in $\mathbb{R}^{d+2}$ (using the affine basis $\boldsymbol{e}_{2, i}, 1 \leq i \leq 3$ ). Let $\boldsymbol{\gamma}_{i}\left(t_{i, j_{i, 1}}\right), \boldsymbol{\gamma}\left(t_{i, j_{i, 2}}\right), \ldots, \boldsymbol{\gamma}_{i}\left(t_{i, j_{i, k_{i}}}\right)$, be the vertices in $U$, and $\boldsymbol{\beta}_{i}\left(t_{i, j_{i, 1}}\right), \boldsymbol{\beta}_{i}\left(t_{i, j_{i, 2}}\right), \ldots, \boldsymbol{\beta}_{i}\left(t_{i, j_{i, k_{i}}}\right)$, be their corresponding vertices in $\mathcal{U}$, where $j_{i, 1}<j_{i, 2}<\ldots<j_{i, k_{i}}$ for all $1 \leq i \leq 3$. Let $\boldsymbol{x}=\left(x_{1}, x_{2}, \ldots, x_{d+2}\right)$ and define the $(d+3) \times(d+3)$ determinant $H_{\mathcal{U}}(\boldsymbol{x})$ as follows:

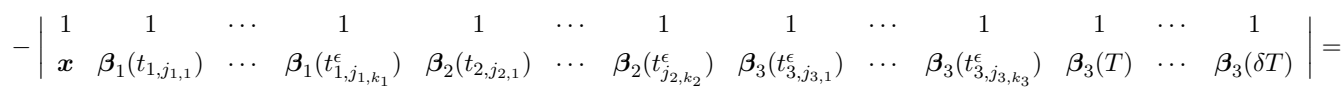

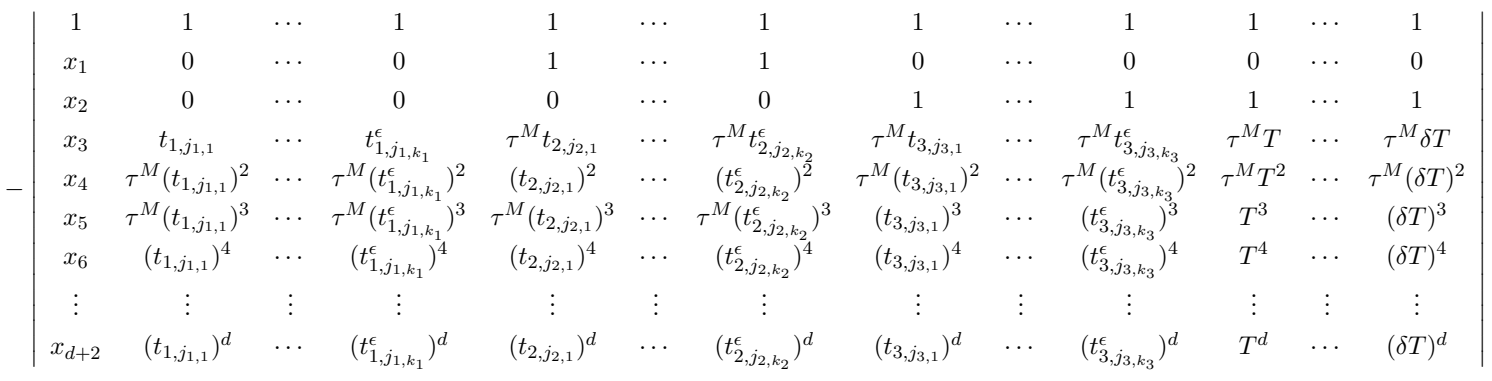

We can alternatively describe $H_{\mathcal{U}}(\boldsymbol{x})$ as follows:

(i) The first column of $H_{\mathcal{U}}(\boldsymbol{x})$ is $\left(\begin{array}{l}1 \\ \boldsymbol{x}\end{array}\right)$.

(ii) For $i$ ranging from 1 to 3 , and for $\lambda$ ranging from 1 to $k_{i}$, the next $k_{i}$ pairs of columns of $H_{\mathcal{U}}(\boldsymbol{x})$ are $\left(\begin{array}{c}1 \\ \boldsymbol{\beta}_{i}\left(t_{i, j_{i, \lambda}}\right)\end{array}\right)$ and $\left(\begin{array}{c}1 \\ \boldsymbol{\beta}_{i}\left(t_{i, j_{i, \lambda}}^{\epsilon}\right)\end{array}\right)$.

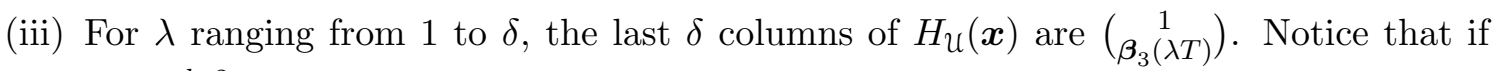
$k=\left\lfloor\frac{d+2}{2}\right\rfloor$ and $d$ is even, this category of columns of $H_{\mathcal{U}}(\boldsymbol{x})$ does not exist. 
The equation $H_{\mathcal{U}}(\boldsymbol{x})=0$ is the equation of a hyperplane in $\mathbb{R}^{d+2}$ that passes through the points in $\mathcal{U}$. Recall that $\mathcal{V}_{[3]}=\mathcal{V}_{1} \cup \mathcal{V}_{2} \cup \mathcal{V}_{3}$. We are going to show that, for any choice of $\mathcal{U}$, and for all vertices $\boldsymbol{v}$ in $\mathcal{V}_{[3]} \backslash \mathcal{U}$, we have $H_{\mathcal{U}}(\boldsymbol{v})>0$ for sufficiently small $\tau$.

Suppose we have some vertex $\boldsymbol{v} \in \mathcal{V}_{[3]} \backslash \mathcal{U}$. Then, $\boldsymbol{v}=\boldsymbol{\beta}_{s}\left(t_{s, \lambda}\right), t_{s, \lambda}=x_{s, \lambda} \tau^{\nu_{s}}$, for some $1 \leq \lambda \leq n_{s}$ and $1 \leq s \leq 3$, such that $\lambda \notin\left\{j_{s, 1}, j_{s, 2}, \ldots, j_{s, k_{s}}\right\}$. Then we can transform $H_{\mathcal{U}}(\boldsymbol{v})$ in the form of the determinant $E_{n, m, k}(\tau ; \boldsymbol{\mu})$ of Lemma 17 , by subtracting the second and third row of $H_{\mathcal{U}}(\boldsymbol{v})$ from its first row and shifting the first column of $H_{\mathcal{U}}(\boldsymbol{v})$ to the right via an even number of column swaps. More precisely, we need to shift the first column of $H_{\mathcal{U}}(\boldsymbol{v})$ to the right so that the values $t_{s, \lambda}, t_{s, j_{s, 1}}, t_{s, j_{s, 1}}^{\epsilon}, t_{s, j_{s, 2}}, t_{s, j_{s, 2}}^{\epsilon}, \ldots, t_{s, j_{s, k_{s}}}, t_{s, j_{s, k_{s}}}^{\epsilon}$, appear consecutively in the columns of $H_{\mathfrak{U}}(\boldsymbol{v})$ and in increasing order. To do that we always need an even number of column swaps, due to the way we have chosen $\epsilon$.

Now, suppose that $\boldsymbol{v} \in \mathcal{V}_{1}$. Then $H_{\mathcal{U}}(\boldsymbol{v})$ is in the form of the determinant $E_{n, m, k}(\tau ; \boldsymbol{\mu})$ of Lemma 17, where $n \leftarrow 2 k_{1}+1, m \leftarrow 2 k_{2}, k \leftarrow 2 k_{3}+\delta, l \leftarrow d+3$, and $\boldsymbol{\mu} \leftarrow$ $(0,0,0,1,2, \ldots, d)$. Obviously, $M \geq 2|\boldsymbol{\mu}|=d(d+1)$. Applying now Lemma 17, we deduce that $H_{\mathcal{U}}(\boldsymbol{v})$ can be written as:

$$
H_{u}(\boldsymbol{v})=C^{\prime} \tau^{\xi}+\Theta\left(\tau^{\xi+1}\right), \quad \xi=4+2 \sum_{i=7}^{2 k_{1}+5}(i-3)+\sum_{i=2 k_{1}+6}^{2 k_{1}+2 k_{2}+3}(i-3),
$$

where $C^{\prime}$ is a positive constant independent of $\tau$. The asymptotic estimate above implies that $H_{\mathcal{U}}(\boldsymbol{v})>0$, for sufficiently small $\tau$.

The remaining cases, i.e., the cases $\boldsymbol{v} \in \mathcal{V}_{2}$ and $\boldsymbol{v} \in \mathcal{V}_{3}$, are completely analogous and we omit them. We thus conclude that, for any specific choice of $U$, and for any specific vertex $\boldsymbol{v} \in \mathcal{V}_{[3]} \backslash \mathcal{U}$, there exists some $\tau_{\boldsymbol{v}}, \mathfrak{u}>0$ (cf. Lemma 17 in Section B of the Appendix) that depends on $\boldsymbol{v}$ and $\mathcal{U}$, such that for all $\tau \in\left(0, \tau_{\boldsymbol{v}}, \mathrm{u}\right)$ we have $H_{\mathcal{U}}(\boldsymbol{v})>0$. For each $k$ with $3 \leq k \leq\left\lfloor\frac{d+2}{2}\right\rfloor$, the number of the sets $\mathcal{U}$ of size $k$ containing at least one vertex from each $\mathcal{V}_{i}, 1 \leq i \leq 3$, is

$$
\left(\begin{array}{c}
n_{1}+n_{2}+n_{3} \\
k
\end{array}\right)-\left(\begin{array}{c}
n_{1}+n_{2} \\
k
\end{array}\right)-\left(\begin{array}{c}
n_{1}+n_{3} \\
k
\end{array}\right)-\left(\begin{array}{c}
n_{2}+n_{3} \\
k
\end{array}\right)+\left(\begin{array}{c}
n_{1} \\
k
\end{array}\right)+\left(\begin{array}{c}
n_{2} \\
k
\end{array}\right)+\left(\begin{array}{c}
n_{3} \\
k
\end{array}\right)=\sum_{\emptyset \subset S \subseteq[3]}(-1)^{3-|S|}\left(\begin{array}{c}
n_{S} \\
k
\end{array}\right) .
$$

For each such subset $\mathcal{U}$ we need to consider the $\left(n_{1}+n_{2}+n_{3}-k\right)$ vertices in $\mathcal{V}_{[3]} \backslash \mathcal{U}$, therefore it suffices to consider a positive value $\hat{\tau}_{[3]}$ for $\tau$ that is small enough, so that all

$$
\sum_{k=3}^{\left\lfloor\frac{d+1}{2}\right\rfloor}\left(n_{1}+n_{2}+n_{3}-k\right) \sum_{\emptyset \subset S \subseteq[3]}(-1)^{3-|S|}\left(\begin{array}{c}
n_{S} \\
k
\end{array}\right),
$$

possible determinants $H_{\mathcal{U}}(\boldsymbol{v})$ are strictly positive. For $\tau \leftarrow \hat{\tau}_{[3]}$, our analysis above immediately implies that for each set $\mathcal{U}$ the equation $H_{\mathcal{U}}(\boldsymbol{x})=0, \boldsymbol{x} \in \mathbb{R}^{d+2}$, is the equation of a supporting hyperplane for $\mathcal{C}$ passing through the vertices of $\mathcal{U}$, and those only. In other words, every set $\mathcal{U}$, of $k$ vertices, for $3 \leq k \leq\left\lfloor\frac{d+2}{2}\right\rfloor$, with at least one vertex from each $\mathcal{V}_{i}$, $1 \leq i \leq 3$, defines a $(k-1)$-face of $\mathcal{C}$, which means that

$$
f_{k-1}\left(\mathcal{F}_{[3]}\right)=\sum_{\emptyset \subset S \subseteq R}(-1)^{3-|S|}\left(\begin{array}{c}
n_{S} \\
k
\end{array}\right), \quad \text { for all } 3 \leq k \leq\left\lfloor\frac{d+2}{2}\right\rfloor .
$$


Relation (55) now immediately follows from Lemmas 13 and 14. First choose a value $\tau^{\star}$ for $\tau$, smaller that $\hat{\tau}_{R}$, for all $2 \leq|R| \leq 3$. Then for this value of $\tau$, the results of both Lemma 13 and Lemma 14 hold true. Moreover, since $P_{1}, P_{2}$ and $P_{3}$ are $\left\lfloor\frac{d}{2}\right\rfloor$-neighborly for any $\tau>0$, and since $f_{-1}\left(\mathcal{F}_{R}\right)=(-1)^{|R|-1}$, for all $\emptyset \subset R \subseteq[3]$, while $f_{k-1}\left(\mathcal{F}_{R}\right)=0$, for all $1 \leq k \leq|R|$, we conclude that, for $\tau \equiv \tau^{\star}$, relations (55) hold.

Based on the analysis above, as well as the analysis in Section 6.1, we conclude that the upper bounds stated in Theorem 12 are actually tight for any $d \geq 2$. This establishes Theorem 1, stated in the introductory section of the paper.

\section{Conclusion}

In this paper we have computed the maximum number of $k$-faces, $f_{k}\left(P_{1}+P_{2}+P_{3}\right), 0 \leq k \leq$ $d-1$, of the Minkowski sum of three $d$-polytopes $P_{1}, P_{2}$ and $P_{3}$ in $\mathbb{R}^{d}$ as a function of the number of their vertices $n_{1}, n_{2}$ and $n_{3}$. When $d=2$ our expressions reduce to known tight bounds, while for $d=3$ we show the tightness of our upper bounds by exploiting results from [10] and [30]. In four or more dimensions we present a novel construction that achieves the upper bounds: we consider the $d$-dimensional moment-like curves:

$$
\begin{aligned}
& \gamma_{1}(t)=\left(t, \zeta t^{2}, \zeta t^{3}, t^{4}, \ldots, t^{d}\right), \\
& \gamma_{2}(t)=\left(\zeta t, t^{2}, \zeta t^{3}, t^{4}, \ldots, t^{d}\right), \\
& \gamma_{3}(t)=\left(\zeta t, \zeta t^{2}, t^{3}, t^{4}, \ldots, t^{d}\right),
\end{aligned}
$$

and we show that our maximal values are attained when $P_{i}$ is the $d$-polytope with vertex set

$$
V_{i}=\left\{\gamma_{i}\left(x_{i, 1} \tau^{\star}\right), \gamma_{i}\left(x_{i, 2} \tau^{\star}\right), \ldots, \gamma_{i}\left(x_{i, n_{i}} \tau^{\star}\right)\right\}, \quad i=1,2,3,
$$

with $0<x_{i, 1}<x_{i, 2}<\cdots<x_{i, n_{i}}$ and $\zeta=\left(\tau^{\star}\right)^{M}$. The parameter value $\tau^{\star}$ is a sufficiently small positive number, while $M$ is chosen sufficiently large.

Our ultimate goal has been to extend our technique for the Minkowski sum of $r d$ polytopes in $\mathbb{R}^{d}$, for $r \geq 4$ and $d \geq 3$. Towards this direction, we can extend our methodology and tools so as to prove relations for $r$ polytopes that generalize certain relations that hold true for two or three polytopes. For example, relation (13) in Lemma 4 generalizes to:

$$
h_{k}\left(\mathcal{K}_{R}\right)=\sum_{\emptyset \subset S \subseteq R} g_{k}^{(|R|-|S|)}\left(\mathcal{F}_{S}\right), \quad 0 \leq k \leq d+|R|-1, \quad \emptyset \subset R \subseteq[r],
$$

where $[r]=\{1,2, \ldots, r\}$. The Dehn-Sommerville-like equations in the same lemma (cf. rel. (15)) generalize to:

$$
h_{d+|R|-1-k}\left(\mathcal{F}_{R}\right)=h_{k}\left(\mathcal{K}_{R}\right), \quad 0 \leq k \leq d+|R|-1, \quad \emptyset \subset R \subseteq[r],
$$

where $\mathcal{F}_{R}$ and $\mathcal{K}_{R}$ are defined as in Section 2. Notice that, since for $r=1$ we have $\mathcal{F}_{[1]} \equiv \mathcal{K}_{[1]} \equiv \partial P_{1}$, the equations in (59) reduce to the well-known Dehn-Sommerville equations for a simplicial $d$-polytope. We can also obtain a generalization of relation (14). Let $\mathcal{Q}_{R}$ be the simplicial $(d+|R|-1)$-sphere we get by performing stellar subdivisions on 
the non-simplicial faces of the Cayley polytope of the polytopes in $R, \emptyset \subset R \subseteq[r]$. For all $0 \leq k \leq d+|R|-1$, we can obtain the following two expressions relating the $h$-vector elements of $\partial \mathcal{Q}_{R}$ with those of $\mathcal{F}_{S}$ and $\mathcal{K}_{S}, \emptyset \subset S \subseteq R$ :

$$
\begin{aligned}
& h_{k}\left(\partial \mathcal{Q}_{R}\right)=h_{k}\left(\mathcal{F}_{R}\right)+\sum_{\emptyset \subset S \subset R} \sum_{i=0}^{|R|-|S|-1} E_{|R|-|S|, i} h_{k-i}\left(\mathcal{F}_{S}\right), \\
& h_{k}\left(\partial \mathcal{Q}_{R}\right)=h_{k}\left(\mathcal{K}_{R}\right)+\sum_{\emptyset \subset S \subset R} \sum_{i=0}^{|R|-|S|-1} E_{|R|-|S|, i} h_{k-1-i}\left(\mathcal{K}_{S}\right),
\end{aligned}
$$

where $E_{m, k}, m \geq k+1>0$, are the Eulerian numbers $[13,1]$ :

$$
E_{m, k}=\sum_{i=0}^{k}(-1)^{i}\left(\begin{array}{c}
m+1 \\
i
\end{array}\right)(k+1-i)^{m}, \quad m \geq k+1>0 .
$$

A recurrence relation similar to (37) in Lemma 8 may also be obtained. More precisely, the following recurrence relation holds for all $0 \leq k \leq d+|R|-2$, where $\emptyset \subset R \subseteq[r]$ :

$$
h_{k+1}\left(\mathcal{F}_{R}\right) \leq \frac{n_{R}-d-|R|+1+k}{k+1} h_{k}\left(\mathcal{F}_{R}\right)+\sum_{i=1}^{|R|} \frac{n_{i}}{k+1} g_{k}\left(\mathcal{F}_{R \backslash\{i\}}\right), \quad n_{R}=\sum_{i \in R} n_{i} .
$$

Using this recurrence relation we can inductively obtain the following upper bounds for the elements of $\boldsymbol{h}\left(\mathcal{F}_{R}\right), \emptyset \subset R \subseteq[r]$ :

$$
h_{k}\left(\mathcal{F}_{R}\right) \leq \sum_{\emptyset \subset S \subseteq R}(-1)^{|R|-|S|}\left(\begin{array}{c}
n_{S}-d-|R|+k \\
k
\end{array}\right), \quad 0 \leq k \leq d+|R|-1 .
$$

These bounds can be shown to be tight for all $k$ with $0 \leq k \leq\left\lfloor\frac{d+|R|-1}{2}\right\rfloor$. Obtaining tight upper bounds for $\boldsymbol{h}\left(\mathcal{K}_{R}\right)$, when $|R| \geq 4$ turns out to be a bit more involved.

The bounds presented in this paper refer to polytopes of the same dimension. We would like to derive refined bounds in the special case of simple polytopes of the same dimension. Finally, a similar problem is to express the number of $k$-faces of the Minkowski sum of $r d$-polytopes in terms of the number of facets of these polytopes. Results in this direction are known for $d=2$ and $d=3$ only. We would like to derive such expressions for any $d \geq 4$ and any number, $r$, of summands.

Current state of the art. In a recent arXiv report [2], Adiprasito and Sanyal have followed the high-level proof logic presented in this paper (as well as in [19], as it is the same logic) to completely resolve what should now be called the Upper Bound Theorem for Minkowski sums. They have proven upper bounds, in terms of the number of vertices of the polytopes, for any number of summands, of the same or different dimensions. Unlike our approach which is entirely geometric, they have used tools from Combinatorial Commutative Algebra to prove relations (59) and (60). This is achieved via a new theory they have developed, called the relative Stanley-Reisner theory for simplicial complexes. Their theory has been 
applied to $\mathcal{K}_{R}$ and $\mathcal{F}_{R}$, called, in their terminology, the Cayley and relative Cayley complexes, respectively. Tight upper bounds for $\boldsymbol{h}\left(\mathcal{K}_{R}\right)$, when $|R| \geq 4$, have been obtained through clever algebraic manipulation of the $h$-vector of $\mathcal{K}_{R}$. One interesting point to make is that the general upper bounds are explicit, but not in closed form: for $|R| \geq 4$ the upper bounds for $\boldsymbol{h}\left(\mathcal{F}_{R}\right)$ and $\boldsymbol{h}\left(\mathcal{K}_{R}\right)$ are expressed through functions that are recursive in $|R|$.

In another very recent arXiv report [20], Karavelas and Tzanaki generalize the geometric approach presented in this paper for three summands to the case of $r$ summands, when $1<r<d$.

\section{Acknowledgements}

The work in this paper has been partially supported by the FP7-REGPOT-2009-1 project "Archimedes Center for Modeling, Analysis and Computation" (under grant agreement $\mathrm{n}^{\mathrm{o}} 245749$ ), and has been co-financed by the European Union (European Social Fund ESF) and Greek national funds through the Operational Program "Education and Lifelong Learning" of the National Strategic Reference Framework (NSRF) - Research Funding Program: THALIS - UOA (MIS 375891).

We would like to thank the anonymous reviewers for useful suggestions and comments that have helped improve the exposition of the material in the paper.

\section{References}

[1] The integer sequence A008292 (Eulerian numbers). The On-Line Encyclopedia of Integer Sequences.

http://oeis.org/A008292.

[2] Karim A. Adiprasito and Raman Sanyal. Relative Stanley-Reisner theory and Upper Bound Theorems for Minkowski sums, December 2014. arXiv:1405.7368v3 [math. CO].

[3] H. Bruggesser and P. Mani. Shellable decompositions of cells and spheres. Math. Scand., 29:197-205, 1971.

http: //www.mscand.dk/article.php?id=2034.

[4] David A. Cox, John Little, and Donal O'Shea. Using Algebraic Geometry, volume 185 of Graduate Texts in Mathematics. Springer, New York, 2nd edition, 2005.

[5] Mark de Berg, Marc van Kreveld, Mark Overmars, and Otfried Schwarzkopf. Computational Geometry: Algorithms and Applications. Springer-Verlag, Berlin, Germany, 2nd edition, 2000.

[6] G. Ewald and G. C. Shephard. Stellar Subdivisions of Boundary Complexes of Convex Polytopes. Mathematische Annalen, 210:7-16, 1974.

http://dx.doi.org/10.1007/BF01344542. 
[7] Efi Fogel, Dan Halperin, and Christophe Weibel. On the Exact Maximum Complexity of Minkowski Sums of Polytopes. Discrete Comput. Geom., 42:654-669, 2009.

http://dx.doi.org/10.1007/s00454-009-9159-1.

[8] Efraim Fogel. Minkowski Sum Construction and other Applications of Arrangements of Geodesic Arcs on the Sphere. PhD thesis, Tel-Aviv University, October 2008.

http://www.cs.tau.ac.il/thesis/thesis/Fogel-Efraim-PhD.pdf.

[9] Komei Fukuda. From the zonotope construction to the Minkowski addition of convex polytopes. J. Symb. Comput., 38:1261-1272, 2004.

http://dx.doi.org/10.1016/j.jsc.2003.08.007.

[10] Komei Fukuda and Christophe Weibel. $f$-vectors of Minkowski Additions of Convex Polytopes. Discrete Comput. Geom., 37(4):503-516, 2007.

http://dx.doi.org/10.1007/s00454-007-1310-2.

[11] F. R. Gantmacher. The Theory of Matrices, volume I. Chelsea Publishing Co., New York, 1960.

[12] F. R. Gantmacher. Applications of the Theory of Matrices. Dover, Mineola, New York, 2005.

[13] R. L. Graham, D. E. Knuth, and O. Patashnik. Concrete Mathematics. AddisonWesley, Reading, MA, 1989.

[14] Peter Gritzmann and Bernd Sturmfels. Minkowski Addition of Polytopes: Computational Complexity and Applications to Gröbner bases. SIAM J. Disc. Math., 6(2):246269, May 1993.

http://dx.doi.org/10.1137/0406019.

[15] Kenneth Hoffman and Ray Kunze. Linear Algebra. Prentice Hall, 2nd edition, 1971.

[16] Birkett Huber, Jörg Rambau, and Francisco Santos. The Cayley Trick, lifting subdivisions and the Bohne-Dress theorem on zonotopal tilings. J. Eur. Math. Soc., 2(2):179198, June 2000.

http://dx.doi.org/10.1007/s100970050003.

[17] Menelaos I. Karavelas, Christos Konaxis, and Eleni Tzanaki. The maximum number of faces of the Minkowski sum of three convex polytopes. In Proceedings of the 29th Annual ACM Symposium on Computational Geometry (SCG'13), pages 187-196, Rio de Janeiro, Brazil, June 17-20, 2013.

http://doi . acm.org/10.1145/2462356.2462368.

[18] Menelaos I. Karavelas and Eleni Tzanaki. Tight lower bounds on the number of faces of the Minkowski sum of convex polytopes via the Cayley trick, December 2011. arXiv:1112.1535v1 [cs.CG].

[19] Menelaos I. Karavelas and Eleni Tzanaki. The maximum number of faces of the Minkowski sum of two convex polytopes. In Proceedings of the 23rd ACM-SIAM Symposium on Discrete Algorithms (SODA'12), pages 11-28, Kyoto, Japan, January 17-19, 
2012 .

http://doi.acm.org/10.1145/2095116.2095118.

[20] Menelaos I. Karavelas and Eleni Tzanaki. A geometric approach for the upper bound theorem for Minkowki sums of convex polytopes, March 2015. arXiv:1502.02265v2 [cs. CG].

[21] Jean-Claude Latombe. Robot Motion Planning. Kluwer Academic Publishers, Norwell, Massachusetts, USA, 1991.

[22] Ming C. Lin and Dinesh Manocha. Collision and proximity queries. In Jacob E. Goodman and Joseph O'Rourke, editors, Handbook of Discrete and Computational Geometry, chapter 35, pages 787-808. CRC Press, Boca Raton, Florida, 2nd edition, 2004.

[23] Jiří Matoušek. Lectures on Discrete Geometry. Graduate Texts in Mathematics. Springer-Verlag New York, Inc., New York, 2002.

[24] P. McMullen. The maximum numbers of faces of a convex polytope. Mathematika, 17:179-184, 1970.

http://dx.doi.org/10.1112/S0025579300002850.

[25] J. Rosenmüller. Game Theory: Stochastics, information, strategies and Cooperation, volume 25 of Theory and Decision Library, Series C. Kluwer Academic Publishers, Dordrecht, 2000.

[26] Raman Sanyal. Topological obstructions for vertex numbers of Minkowski sums. J. Comb. Theory, Ser. A, 116(1):168-179, 2009.

http://dx.doi.org/10.1016/j.jcta.2008.05.009.

[27] Bernd Sturmfels. Gröbner Bases and Convex Polytopes, volume 8 of Univ. Lectures Series. American Mathematical Society, Providence, Rhode Island, 1996.

[28] Alexander V. Tuzikov, Jos B.T.M. Roerdink, and Henk J.A.M. Heijmans. Similarity measures for convex polyhedra based on Minkowski addition. Pattern Recognition, 33(6):979-995, 2000.

http://dx.doi.org/10.1016/S0031-3203(99)00159-4.

[29] Christophe Weibel. Minkowski Sums of Polytopes: Combinatorics and Computation. PhD thesis, École Polytechnique Fédérale de Lausanne, 2007.

http://dx.doi.org/10.5075/epfl-thesis-3883.

[30] Christophe Weibel. Maximal f-vectors of Minkowski Sums of Large Numbers of Polytopes. Discrete Comput. Geom., 47(3):519-537, April 2012.

http://dx.doi.org/10.1007/s00454-011-9385-1.

[31] Günter M. Ziegler. Lectures on Polytopes, volume 152 of Graduate Texts in Mathematics. Springer-Verlag, New York, 1995. 


\section{A Omitted \& full proofs}

\section{A.1 Omitted \& full proofs of Section 3}

Proof of Lemma 2. The result clearly holds for $m=0$, since:

$$
g_{k}^{(0)}(\mathcal{Y})=h_{k}(\mathcal{Y})=\sum_{i=0}^{0}(-1)^{i}\left(\begin{array}{l}
0 \\
i
\end{array}\right) h_{k-i}(\mathcal{Y}) .
$$

Suppose the relation holds for some $m \geq 0$. We will show it holds for $m+1$. Indeed:

$$
\begin{aligned}
g_{k}^{(m+1)}(\mathcal{Y}) & =g_{k}^{(m)}(\mathcal{Y})-g_{k-1}^{(m)}(\mathcal{Y}) \\
& =\sum_{i=0}^{m}(-1)^{i}\left(\begin{array}{c}
m \\
i
\end{array}\right) h_{k-i}(\mathcal{Y})-\sum_{i=0}^{m}(-1)^{i}\left(\begin{array}{c}
m \\
i
\end{array}\right) h_{k-1-i}(\mathcal{Y}) \\
& =\sum_{i=0}^{m+1}(-1)^{i}\left(\begin{array}{c}
m \\
i
\end{array}\right) h_{k-i}(\mathcal{Y})-\sum_{j=1}^{m+1}(-1)^{j-1}\left(\begin{array}{c}
m \\
j-1
\end{array}\right) h_{k-j}(\mathcal{Y}) \\
& =\sum_{i=0}^{m+1}(-1)^{i}\left(\begin{array}{c}
m \\
i
\end{array}\right) h_{k-i}(\mathcal{Y})-\sum_{j=0}^{m+1}(-1)^{j-1}\left(\begin{array}{c}
m \\
j-1
\end{array}\right) h_{k-j}(\mathcal{Y}) \\
& =\sum_{i=0}^{m+1}(-1)^{i}\left(\begin{array}{c}
m \\
i
\end{array}\right) h_{k-i}(\mathcal{Y})+\sum_{i=0}^{m+1}(-1)^{i}\left(\begin{array}{c}
m \\
i-1
\end{array}\right) h_{k-i}(\mathcal{Y}) \\
& =\sum_{i=0}^{m+1}(-1)^{i}\left[\left(\begin{array}{c}
m \\
i
\end{array}\right)+\left(\begin{array}{c}
m \\
i-1
\end{array}\right)\right] h_{k-i}(\mathcal{Y}) \\
& =\sum_{i=0}^{m+1}(-1)^{i}\left(\begin{array}{c}
m+1 \\
i
\end{array}\right) h_{k-i}(\mathcal{Y}) .
\end{aligned}
$$

Proof of Lemma 3. By replacing $h_{k-\nu-j}(\mathcal{Y})$ from its defining equation, we get:

$$
\begin{aligned}
g_{k-\nu}^{(D-\delta-\nu)}(\mathcal{Y}) & =\sum_{j=0}^{D-\delta-\nu}(-1)^{j}\left(\begin{array}{c}
D-\delta-\nu \\
j
\end{array}\right) h_{k-\nu-j}(\mathcal{Y}) \\
& =\sum_{j=0}^{D-\delta-\nu}(-1)^{j}\left(\begin{array}{c}
D-\delta-\nu \\
j
\end{array}\right) \sum_{i=0}^{\delta+1}(-1)^{k-\nu-j-i}\left(\begin{array}{c}
\delta+1-i \\
\delta+1-k+\nu+j
\end{array}\right) f_{i-1}(\mathcal{Y}) \\
& =\sum_{j=0}^{D-\delta-\nu}(-1)^{j}\left(\begin{array}{c}
D-\delta-\nu \\
j
\end{array}\right) \sum_{i=0}^{D+1}(-1)^{k-\nu-j-i}\left(\begin{array}{c}
\delta+1-i \\
\delta+1-k+\nu+j
\end{array}\right) f_{i-1}(\mathcal{Y}) \\
& =\sum_{i=0}^{D+1}(-1)^{k-\nu-i} f_{i-1}(\mathcal{Y}) \sum_{j=0}^{D-\delta-\nu}\left(\begin{array}{c}
D-\delta-\nu \\
j
\end{array}\right)\left(\begin{array}{c}
\delta+1-i \\
k-\nu-i-j
\end{array}\right) \\
= & \sum_{i=0}^{D+1}(-1)^{k-\nu-i}\left(\begin{array}{c}
D+1-\nu-i \\
k-\nu-i
\end{array}\right) f_{i-1}(\mathcal{Y})
\end{aligned}
$$




$$
\begin{aligned}
= & \sum_{i=0}^{k-\nu}(-1)^{k-\nu-i}\left(\begin{array}{c}
D+1-\nu-i \\
D+1-k
\end{array}\right) f_{i-1}(\mathcal{Y}) \\
= & \sum_{j=\nu}^{k}(-1)^{k-j}\left(\begin{array}{c}
D+1-j \\
D+1-k
\end{array}\right) f_{j-\nu-1}(\mathcal{Y}) \\
= & \sum_{j=0}^{D+1}(-1)^{k-j}\left(\begin{array}{c}
D+1-j \\
D+1-k
\end{array}\right) f_{j-\nu-1}(\mathcal{Y}) \\
= & \mathcal{S}_{k}(\mathcal{Y} ; D, \nu),
\end{aligned}
$$

where:

- in order to go from (61) to (62), we used that $\left(\begin{array}{c}\delta+1-i \\ \delta+1-k+\nu+j\end{array}\right)=0$ for $i>\delta+1$,

- in order to go from (63) to (64), we used the combinatorial identity:

$$
\sum_{i=0}^{n}\left(\begin{array}{c}
n \\
i
\end{array}\right)\left(\begin{array}{c}
m \\
k-i
\end{array}\right)=\sum_{i=0}^{k}\left(\begin{array}{c}
n \\
i
\end{array}\right)\left(\begin{array}{c}
m \\
k-i
\end{array}\right)=\left(\begin{array}{c}
n+m \\
k
\end{array}\right),
$$

with $n \leftarrow D-\delta-\nu, m \leftarrow \delta+1-i, i \leftarrow j, k \leftarrow k-\nu-i$,

- in order to go from (64) to (65), we used that $\left(\begin{array}{c}D+1-\nu-i \\ k-\nu-i\end{array}\right)=0$ for $i>k-\nu$, and that $\left(\begin{array}{c}D+1-\nu-i \\ k-\nu-i\end{array}\right)=\left(\begin{array}{c}D+1-\nu-i \\ (D+1-\nu-i)-(k-\nu-i)\end{array}\right)=\left(\begin{array}{c}D+1-\nu-i \\ D+1-k\end{array}\right)$, and, finally,

- in order to go from $(66)$ to $(67)$, we used that $f_{j-\nu-1}(\mathcal{Y})=0$ for $j<\nu$ (i.e., for $j-\nu-1<-1$ ), and that $\left(\begin{array}{c}D+1-j \\ D+1-k\end{array}\right)=0$ for $j>k$.

\section{A.2 Omitted \& full proofs of Section 4}

Proof of Lemma 6. Using relation (14), and after rearranging the terms, the left hand side of relation (19) becomes:

$$
\begin{aligned}
& \overbrace{(k+1) h_{k+1}\left(\mathcal{F}_{[3]}\right)+(d+2-k) h_{k}\left(\mathcal{F}_{[3]}\right)}^{T_{1}} \overbrace{\sum_{R \in X_{2}}\left[(k+1) h_{k+1}\left(\mathcal{F}_{R}\right)+(d+2-k) h_{k}\left(\mathcal{F}_{R}\right)\right]}^{T_{2}} \\
& +\overbrace{\sum_{R \in X_{1}}\left[(k+1) h_{k+1}\left(\mathcal{F}_{R}\right)+(d+2-k) h_{k}\left(\mathcal{F}_{R}\right)\right]}^{T_{3}} \\
& +\overbrace{\sum_{R \in X_{1}}\left[(k+1) h_{k}\left(\mathcal{F}_{R}\right)+(d+2-k) h_{k-1}\left(\mathcal{F}_{R}\right)\right]}^{T_{4}} .
\end{aligned}
$$


We are going to analyze each term in the expression above separately. For any $R \in X_{2}$ : (i) the relation at the top of page 18 in [19, Lemma 3.2], (ii) relations (13), with $R \in X_{2}$, and (iii) relation (3.9) in [19], give:

$$
\begin{aligned}
(k+1) h_{k+1}\left(\mathcal{F}_{R}\right)+(d+1-k) h_{k}\left(\mathcal{F}_{R}\right) & =\sum_{i \in R} \sum_{v \in \mathcal{V}_{i}}\left[h_{k}\left(\mathcal{K}_{R} / v\right)-g_{k}\left(\mathcal{K}_{\{i\}} / v\right)\right] \\
& =\sum_{v \in \mathcal{V}_{R}} h_{k}\left(\mathcal{K}_{R} / v\right)-\sum_{\emptyset \subset S \subset R} \sum_{v \in \mathcal{V}_{S}} g_{k}\left(\mathcal{K}_{S} / v\right) .
\end{aligned}
$$

Hence term $T_{2}$ can be rewritten as:

$$
\begin{aligned}
T_{2} & =\sum_{R \in \mathcal{X}_{2}} h_{k}\left(\mathcal{F}_{R}\right)+\sum_{R \in \mathcal{X}_{2}} \sum_{v \in \mathcal{V}_{R}} h_{k}\left(\mathcal{K}_{R} / v\right)-\sum_{R \in \mathcal{X}_{2}} \sum_{\emptyset \subset S \subset R} \sum_{v \in \mathcal{V}_{S}} g_{k}\left(\mathcal{K}_{S} / v\right) \\
& =\overbrace{\sum_{R \in \mathcal{X}_{2}} h_{k}\left(\mathcal{F}_{R}\right)}^{T_{5}}+\overbrace{\sum_{R \in \mathcal{X}_{2}} \sum_{v \in \mathcal{V}_{R}} h_{k}\left(\mathcal{K}_{R} / v\right)}^{T_{6}}-\overbrace{2 \sum_{\sum_{R \in \mathcal{X}_{1}} \sum_{v \in \mathcal{V}_{R}} g_{k}\left(\mathcal{K}_{R} / v\right)}^{T_{7}} .}
\end{aligned}
$$

Applying relation (18) to the $(d-1)$-complex $\mathcal{F}_{R}, R \in X_{1}$, and using the identity $\mathcal{F}_{R} \equiv \mathcal{K}_{R}\left(\equiv \partial P_{R}\right)$, we derive the following expressions:

$$
\begin{aligned}
(k+1) h_{k+1}\left(\mathcal{F}_{R}\right)+(d-k) h_{k}\left(\mathcal{F}_{R}\right) & =\sum_{v \in \mathcal{V}_{R}} h_{k}\left(\mathcal{K}_{R} / v\right), \\
k h_{k}\left(\mathcal{F}_{R}\right)+(d-(k-1)) h_{k-1}\left(\mathcal{F}_{R}\right) & =\sum_{v \in \mathcal{V}_{R}} h_{k-1}\left(\mathcal{K}_{R} / v\right),
\end{aligned}
$$

which, in turn yield the following expansions for $T_{3}$ and $T_{4}$ :

$$
\begin{aligned}
T_{3} & =\overbrace{\sum_{R \in X_{1}} \sum_{v \in \mathcal{V}_{R}} h_{k}\left(\mathcal{K}_{R} / v\right)}^{T_{8}}+\overbrace{2 \sum_{R \in \mathcal{X}_{1}} h_{k}\left(\mathcal{F}_{R}\right)}^{T_{9}}, \\
T_{4} & =\overbrace{\sum_{R \in X_{1}} \sum_{v \in \mathcal{V}_{R}} h_{k-1}\left(\mathcal{K}_{R} / v\right)}^{T_{10}}+\overbrace{\sum_{R \in X_{1}}\left[h_{k}\left(\mathcal{F}_{R}\right)+h_{k-1}\left(\mathcal{F}_{R}\right)\right]}^{T_{11}} .
\end{aligned}
$$

On the other hand, utilizing the expressions in Lemma 5, we arrive at the following expansion for the right-hand side of (19):

$$
\begin{aligned}
& \sum_{i=1}^{3} \sum_{v \in \mathcal{V}_{i}}\left[h_{k}\left(\mathcal{K}_{[3]} / v\right)+\sum_{\{i\} \subseteq R \subset[3]} h_{k-1}\left(\mathcal{K}_{R} / v\right)+h_{k-2}\left(\mathcal{K}_{\{i\}} / v\right)\right] \\
& +\sum_{R \in \mathcal{X}_{1}}\left[h_{k}\left(\mathcal{F}_{R}\right)+h_{k-1}\left(\mathcal{F}_{R}\right)\right]+\sum_{R \in \mathcal{X}_{2}} \sum_{\emptyset \subset S \subseteq R} h_{k}\left(\mathcal{F}_{S}\right) .
\end{aligned}
$$

Since

$$
\sum_{i=1}^{3} \sum_{v \in \mathcal{V}_{i}} h_{k}\left(\mathcal{K}_{[3]} / v\right)=\sum_{v \in \mathcal{V}_{[3]}} h_{k}\left(\mathcal{K}_{[3]} / v\right)
$$




$$
\begin{aligned}
\sum_{i=1}^{3} \sum_{v \in \mathcal{V}_{i}} \sum_{\{i\} \subseteq R \subset[3]} h_{k-1}\left(\mathcal{K}_{R} / v\right) & =\sum_{R \in \mathcal{X}_{2}} \sum_{v \in \mathcal{V}_{R}} h_{k-1}\left(\mathcal{K}_{R} / v\right)+\sum_{R \in \mathcal{X}_{1}} \sum_{v \in \mathcal{V}_{R}} h_{k-1}\left(\mathcal{K}_{R} / v\right), \\
\sum_{i=1}^{3} \sum_{v \in \mathcal{V}_{i}} h_{k-2}\left(\mathcal{K}_{\{i\}} / v\right) & =\sum_{R \in \mathcal{X}_{1}} \sum_{v \in \mathcal{V}_{R}} h_{k-2}\left(\mathcal{K}_{R} / v\right),
\end{aligned}
$$

and

$$
\sum_{R \in \mathcal{X}_{2}} \sum_{\emptyset \subset S \subseteq R} h_{k}\left(\mathcal{F}_{S}\right)=\sum_{R \in \mathcal{X}_{2}} h_{k}\left(\mathcal{F}_{R}\right)+2 \sum_{R \in \mathcal{X}_{1}} h_{k}\left(\mathcal{F}_{R}\right),
$$

the expression in (71) can be rewritten in the following more convenient form:

$$
\begin{aligned}
& \overbrace{\sum_{v \in \mathcal{V}_{[3]} h_{k}\left(\mathcal{K}_{[3]} / v\right)}^{T_{12}}+\overbrace{\sum_{R \in X_{2}} \sum_{v \in \mathcal{V}_{R}} h_{k-1}\left(\mathcal{K}_{R} / v\right)}^{T_{13}}+\overbrace{\sum_{R \in X_{1}} \sum_{v \in \mathcal{V}_{R}}\left[h_{k-1}\left(\mathcal{K}_{R} / v\right)+h_{k-2}\left(\mathcal{K}_{R} / v\right)\right]}^{T_{14}}}^{\overbrace{\sum_{R \in \mathcal{X}_{1}}\left[h_{k}\left(\mathcal{F}_{R}\right)+h_{k-1}\left(\mathcal{F}_{R}\right)\right]+\sum_{R \in X_{2}} h_{k}\left(\mathcal{F}_{R}\right)+2 \sum_{R \in X_{1}} h_{k}\left(\mathcal{F}_{R}\right)}^{T_{15}} .}
\end{aligned}
$$

Solving relation (19) in terms of the term $T_{1}$, we get:

$$
\begin{aligned}
T_{1} & =T_{12}+T_{13}+T_{14}+T_{15}-\left(T_{2}+T_{3}+T_{4}\right) \\
& =T_{12}+T_{13}+T_{14}+T_{15}-\left[\left(T_{5}+T_{6}-T_{7}\right)+\left(T_{8}+T_{9}\right)+\left(T_{10}+T_{11}\right)\right] \\
& =T_{12}+\left(T_{13}-T_{6}\right)+\left(T_{14}+T_{7}-T_{8}-T_{10}\right)+\left(T_{15}-T_{5}-T_{9}-T_{11}\right) \\
& =T_{12}+\left(T_{13}-T_{6}\right)+\left(T_{14}+T_{7}-T_{8}-T_{10}\right),
\end{aligned}
$$

where we used the fact that the terms $T_{5}, T_{9}$ and $T_{11}$ cancel-out with the term $T_{15}$. Observe now that:

$$
T_{13}-T_{6}=\sum_{R \in \mathcal{X}_{2}} \sum_{v \in \mathcal{V}_{R}} h_{k-1}\left(\mathcal{K}_{R} / v\right)-\sum_{R \in \mathcal{X}_{2}} \sum_{v \in \mathcal{V}_{R}} h_{k}\left(\mathcal{K}_{R} / v\right)=-\sum_{R \in \mathcal{X}_{2}} \sum_{v \in \mathcal{V}_{R}} g_{k}\left(\mathcal{K}_{R} / v\right),
$$

while

$$
\begin{aligned}
T_{14}+T_{7}-T_{8}-T_{10}= & \sum_{R \in \mathcal{X}_{1}} \sum_{v \in \mathcal{V}_{R}}\left[h_{k-1}\left(\mathcal{K}_{R} / v\right)+h_{k-2}\left(\mathcal{K}_{R} / v\right)\right]+2 \sum_{R \in \mathcal{X}_{1}} \sum_{v \in \mathcal{V}_{R}} g_{k}\left(\mathcal{K}_{R} / v\right) \\
& -\sum_{R \in \mathcal{X}_{1}} \sum_{v \in \mathcal{V}_{R}} h_{k}\left(\mathcal{K}_{R} / v\right)-\sum_{R \in \mathcal{X}_{1}} \sum_{v \in \mathcal{V}_{R}} h_{k-1}\left(\mathcal{K}_{R} / v\right) \\
= & \sum_{R \in X_{1}} \sum_{v \in \mathcal{V}_{R}}\left\{h_{k-1}\left(\mathcal{K}_{R} / v\right)+h_{k-2}\left(\mathcal{K}_{R} / v\right)+2\left[h_{k}\left(\mathcal{K}_{R} / v\right)-h_{k-1}\left(\mathcal{K}_{R} / v\right)\right]\right. \\
= & \sum_{R \in X_{1}} \sum_{v \in \mathcal{V}_{R}}\left[h_{k}\left(\mathcal{K}_{R} / v\right)-2 h_{k-1}\left(\mathcal{K}_{R} / v\right)+\mathcal{K}_{R-2}(v)\right\}
\end{aligned}
$$




$$
=\sum_{R \in \mathcal{X}_{1}} \sum_{v \in \mathcal{V}_{R}} g_{k}^{(2)}\left(\mathcal{K}_{R} / v\right) .
$$

Hence,

$$
\begin{aligned}
T_{1} & =\sum_{v \in \mathcal{V}_{[3]}} h_{k}\left(\mathcal{K}_{[3]} / v\right)-\sum_{R \in \mathcal{X}_{2}} \sum_{v \in \mathcal{V}_{R}} g_{k}\left(\mathcal{K}_{R} / v\right)+\sum_{R \in \mathcal{X}_{1}} \sum_{v \in \mathcal{V}_{R}} g_{k}^{(2)}\left(\mathcal{K}_{R} / v\right) \\
& =\sum_{\emptyset \subset R \subseteq[3]}(-1)^{3-|R|} \sum_{v \in \mathcal{V}_{R}} g_{k}^{(3-|R|)}\left(\mathcal{K}_{R} / v\right) .
\end{aligned}
$$

Proof of Lemma 8. By Lemma 7, relation (26) yields:

$$
\begin{aligned}
(k+1) h_{k+1}\left(\mathcal{F}_{[3]}\right)+(d+2-k) h_{k}\left(\mathcal{F}_{[3]}\right) & \leq \sum_{\emptyset \subset R \subseteq[3]}(-1)^{3-|R|} n_{R} g_{k}^{(3-|R|)}\left(\mathcal{K}_{R}\right) \\
& =n_{[3]} h_{k}\left(\mathcal{K}_{[3]}\right)-\sum_{R \in \mathcal{X}_{2}} n_{R} g_{k}\left(\mathcal{K}_{R}\right)+\sum_{R \in \mathcal{X}_{1}} n_{R} g_{k}^{(2)}\left(\mathcal{K}_{R}\right)
\end{aligned}
$$

By relation (13) with $R \equiv[3]$, we can write $h_{k}\left(\mathcal{K}_{[3]}\right)$ as:

$$
h_{k}\left(\mathcal{K}_{[3]}\right)=h_{k}\left(\mathcal{F}_{[3]}\right)+\sum_{R \in \mathcal{X}_{2}} g_{k}\left(\mathcal{F}_{R}\right)+\sum_{R \in \mathcal{X}_{1}} g_{k}^{(2)}\left(\mathcal{F}_{R}\right),
$$

whereas from relation (13) for all $R \in X_{2}$ we easily get:

$$
g_{k}\left(\mathcal{K}_{R}\right)=g_{k}\left(\mathcal{F}_{R}\right)+\sum_{\emptyset \subset S \subset R} g_{k}^{(2)}\left(\mathcal{F}_{S}\right) .
$$

Since $\mathcal{K}_{R} \equiv \mathcal{F}_{R}$, for any $R \in \mathcal{X}_{1}$, we can employ relations (73) and (74) to rewrite the right hand side of (72) as follows:

$$
\begin{aligned}
n_{[3]} h_{k}\left(\mathcal{K}_{[3]}\right)-\sum_{R \in \mathcal{X}_{2}} n_{R} g_{k}\left(\mathcal{K}_{R}\right)+\sum_{R \in \mathcal{X}_{1}} n_{R} g_{k}^{(2)}\left(\mathcal{K}_{R}\right) \\
=n_{[3]} h_{k}\left(\mathcal{F}_{[3]}\right)+n_{[3]} \sum_{R \in \mathcal{X}_{2}} g_{k}\left(\mathcal{F}_{R}\right)+n_{[3]} \sum_{R \in \mathcal{X}_{1}} g_{k}^{(2)}\left(\mathcal{F}_{R}\right) \\
-\left[\sum_{R \in \mathcal{X}_{2}} n_{R} g_{k}\left(\mathcal{F}_{R}\right)+\sum_{R \in \mathcal{X}_{2}} n_{R} \sum_{\emptyset \subset S \subset R} g_{k}^{(2)}\left(\mathcal{F}_{S}\right)\right]+\sum_{R \in \mathcal{X}_{1}} n_{R} g_{k}^{(2)}\left(\mathcal{F}_{R}\right) \\
=n_{[3]} h_{k}\left(\mathcal{F}_{[3]}\right)+\sum_{R \in \mathcal{X}_{2}}\left(n_{[3]}-n_{R}\right) g_{k}\left(\mathcal{F}_{R}\right) \\
+\overbrace{\left[n_{[3]} \sum_{i=1}^{3} g_{k}^{(2)}\left(\mathcal{F}_{\{i\}}\right)-\sum_{R \in \mathcal{X}_{2}} n_{R} \sum_{\emptyset \subset S \subset R} g_{k}^{(2)}\left(\mathcal{F}_{S}\right)+\sum_{i=1}^{3} n_{i} g_{k}^{(2)}\left(\mathcal{F}_{\{i\}}\right)\right]}^{[} .
\end{aligned}
$$


Using the identity:

$$
\sum_{R \in \mathcal{X}_{2}} n_{R} \sum_{\emptyset \subset S \subset R} g_{k}^{(2)}\left(\mathcal{F}_{S}\right)=2 \sum_{i=1}^{3} n_{i} g_{k}^{(2)}\left(\mathcal{F}_{\{i\}}\right)+\sum_{i=1}^{3} n_{[3] \backslash\{i\}} g_{k}^{(2)}\left(\mathcal{F}_{\{i\}}\right),
$$

we see that the last term $(\operatorname{term} T)$ in the relation above vanishes:

$$
\begin{aligned}
n_{[3]} & \sum_{i=1}^{3} g_{k}^{(2)}\left(\mathcal{F}_{\{i\}}\right)-\sum_{R \in \mathcal{X}_{2}} n_{R} \sum_{\emptyset \subset S \subset R} g_{k}^{(2)}\left(\mathcal{F}_{S}\right)+\sum_{i=1}^{3} n_{\{i\}} g_{k}^{(2)}\left(\mathcal{F}_{\{i\}}\right) \\
& =n_{[3]} \sum_{i=1}^{3} g_{k}^{(2)}\left(\mathcal{F}_{\{i\}}\right)-\left[2 \sum_{i=1}^{3} n_{i} g_{k}^{(2)}\left(\mathcal{F}_{\{i\}}\right)+\sum_{i=1}^{3} n_{[3] \backslash\{i\}} g_{k}^{(2)}\left(\mathcal{F}_{\{i\}}\right)\right]+\sum_{i=1}^{3} n_{i} g_{k}^{(2)}\left(\mathcal{F}_{\{i\}}\right) \\
& =\sum_{i=1}^{3}\left(n_{[3]}-2 n_{i}-n_{[3] \backslash\{i\}}+n_{i}\right) g_{k}^{(2)}\left(\mathcal{F}_{\{i\}}\right)=0 .
\end{aligned}
$$

Hence, relation (72) simplifies to:

$$
\begin{aligned}
(k+1) h_{k+1}\left(\mathcal{F}_{[3]}\right) & +(d+2-k) h_{k}\left(\mathcal{F}_{[3]}\right) \leq n_{[3]} h_{k}\left(\mathcal{F}_{[3]}\right)+\sum_{R \in \mathcal{X}_{2}}\left(n_{[3]}-n_{R}\right) g_{k}\left(\mathcal{F}_{R}\right) \\
& =n_{[3]} h_{k}\left(\mathcal{F}_{[3]}\right)+\sum_{R \in \mathcal{X}_{2}} n_{[3] \backslash R} g_{k}\left(\mathcal{F}_{R}\right)=n_{[3]} h_{k}\left(\mathcal{F}_{[3]}\right)+\sum_{i=1}^{3} n_{i} g_{k}\left(\mathcal{F}_{[3] \backslash\{i\}}\right),
\end{aligned}
$$

from which we obtain the relation in the statement of the lemma.

\section{A.3 Omitted \& full proofs of Section 5}

Proof of Lemma 11. The bound for $h_{k}\left(\mathcal{K}_{[3]}\right)$ holds as equality for $k=0$, since by relation (13) with $R=[3]$, (see also (73)), we have

$$
\begin{aligned}
h_{0}\left(\mathcal{K}_{[3]}\right) & =h_{0}\left(\mathcal{F}_{[3]}\right)+\sum_{R \in \mathcal{X}_{2}} g_{0}\left(\mathcal{F}_{R}\right)+\sum_{i=1}^{3} g_{0}^{(2)}\left(\partial P_{i}\right) \\
& =1+\sum_{R \in X_{2}}\left[h_{0}\left(\mathcal{F}_{R}\right)-h_{-1}\left(\mathcal{F}_{R}\right)\right]+\sum_{i=1}^{3}\left[h_{0}\left(\partial P_{i}\right)-2 h_{-1}\left(\partial P_{i}\right)+h_{-2}\left(\partial P_{i}\right)\right] \\
& =1+\sum_{R \in X_{2}}[(-1)-0]+\sum_{i=1}^{3}[1-2 \cdot 0+0]=1 .
\end{aligned}
$$

Suppose now that $k \geq 1$. Then, using relations (13) and (40), we get, for $k \geq 1$ :

$$
\begin{aligned}
& h_{k}\left(\mathcal{K}_{[3]}\right)=h_{k}\left(\mathcal{F}_{[3]}\right)+\sum_{R \in X_{2}} g_{k}\left(\mathcal{F}_{R}\right)+\sum_{R \in X_{1}} g_{k}^{(2)}\left(\mathcal{F}_{R}\right) \\
& \quad \leq h_{k}\left(\mathcal{F}_{[3]}\right)+\sum_{R \in X_{2}}\left[\frac{n_{R}-d-3}{k} h_{k-1}\left(\mathcal{F}_{R}\right)+\sum_{i \in R} \frac{n_{R \backslash\{i\}}}{k} g_{k-1}\left(\partial P_{i}\right)\right]+\sum_{i=1}^{3} g_{k}^{(2)}\left(\partial P_{i}\right)
\end{aligned}
$$




$$
=h_{k}\left(\mathcal{F}_{[3]}\right)+\sum_{R \in X_{2}} \frac{n_{R}-d-3}{k} h_{k-1}\left(\mathcal{F}_{R}\right)+\sum_{i=1}^{3}\left[\frac{n_{[3] \backslash\{i\}}}{k} g_{k-1}\left(\partial P_{i}\right)+g_{k}\left(\partial P_{i}\right)-g_{k-1}\left(\partial P_{i}\right)\right],
$$

which finally yields:

$$
h_{k}\left(\mathcal{K}_{[3]}\right) \leq h_{k}\left(\mathcal{F}_{[3]}\right)+\sum_{R \in \mathcal{X}_{2}} \frac{n_{R}-d-3}{k} h_{k-1}\left(\mathcal{F}_{R}\right)+\sum_{i=1}^{3}\left[\frac{n_{[3] \backslash\{i\}}-k}{k} g_{k-1}\left(\partial P_{i}\right)+g_{k}\left(\partial P_{i}\right)\right] .
$$

Since $n_{R}-d-3 \geq 2(d+1)-d-3=d-1>0$, for $R \in \mathcal{X}_{2}$, and $n_{[3 \backslash \backslash\{i\}}-k \geq 2(d+1)-(d+2)=$ $d>0$ for any $0 \leq k \leq d+2$, we can use the upper bounds for $h_{k}\left(\mathcal{F}_{[3]}\right)$ and $h_{k-1}\left(\mathcal{F}_{R}\right), R \in X_{2}$ from Lemma 10 and [19, Lemma 3.3], respectively, in conjunction with the known upper bounds for the elements of the $g$-vector of a $d$-polytope (cf. [31, Corollary 8.38]). More precisely:

$$
\begin{aligned}
h_{k}\left(\mathcal{K}_{[3]}\right) \leq & \sum_{\emptyset \subset S \subseteq[3]}(-1)^{3-|S|}\left(\begin{array}{c}
n_{S}-d-3+k \\
k
\end{array}\right)+\sum_{R \in X_{2}} \frac{n_{R}-d-3}{k}\left[\left(\begin{array}{c}
n_{R}-d-2+k-1 \\
k-1
\end{array}\right)-\sum_{i \in R}\left(\begin{array}{c}
n_{i}-d-2+k-1 \\
k-1
\end{array}\right)\right] \\
& +\sum_{i=1}^{3}\left[\frac{n_{[3] \backslash\{i\}}-k}{k}\left(\begin{array}{c}
n_{i}-d-2+k-1 \\
k-1
\end{array}\right)+g_{k}\left(\partial P_{i}\right)\right] \\
= & \left.\sum_{\emptyset \subset S \subseteq[3]}(-1)^{3-|S|}\left(\begin{array}{c}
n_{S}-d-3+k \\
k
\end{array}\right)+\sum_{R \in \mathcal{R}_{2}} \frac{n_{R}-d-3}{k}\left[\begin{array}{c}
n_{R}-d-3+k \\
k-1
\end{array}\right)-\sum_{i \in R}\left(\begin{array}{c}
n_{i}-d-3+k \\
k-1
\end{array}\right)\right] \\
& +\sum_{i=1}^{3}\left[\frac{n_{[3] \backslash\{i\}}}{k}\left(\begin{array}{c}
n_{i}-d-3+k \\
k-1
\end{array}\right)-\left(\begin{array}{c}
n_{i}-d-3+k \\
k-1
\end{array}\right)+\left(\begin{array}{c}
n_{i}-d-2+k \\
k
\end{array}\right)+g_{k}\left(\partial P_{i}\right)-\left(\begin{array}{c}
n_{i}-d-2+k \\
k
\end{array}\right)\right] \\
= & \left(\begin{array}{l}
n_{[3]}-d-3+k \\
k
\end{array}\right)-\sum_{i=1}^{3}\left(\begin{array}{c}
n_{[3] \backslash\{i\}}-d-3+k \\
k
\end{array}\right)+\sum_{i=1}^{3}\left(\begin{array}{c}
n_{i}-d-3+k \\
k
\end{array}\right) \\
& \left.+\sum_{R \in X_{2}} \frac{n_{R}-d-3}{k}\left[\begin{array}{c}
n_{R}-d-3+k \\
k-1
\end{array}\right)-\sum_{i \in R}\left(\begin{array}{c}
n_{i}-d-3+k \\
k-1
\end{array}\right)\right] \\
& +\sum_{i=1}^{3} \frac{n_{[3] \backslash\{i\}}}{k}\left(\begin{array}{c}
n_{i}-d-3+k \\
k-1
\end{array}\right)+\sum_{i=1}^{3}\left(\begin{array}{c}
n_{i}-d-3+k \\
k
\end{array}\right)+\sum_{i=1}^{3}\left[g_{k}\left(\partial P_{i}\right)-\left(\begin{array}{c}
n_{i}-d-2+k \\
k
\end{array}\right)\right] .
\end{aligned}
$$

From the proof of Lemma 9 it is easy to see that:

$$
\begin{aligned}
& \sum_{R \in X_{2}} \frac{n_{R}-d-3}{k}\left[\left(\begin{array}{c}
n_{R}-d-3+k \\
k-1
\end{array}\right)-\sum_{i \in R}\left(\begin{array}{c}
n_{i}-d-3+k \\
k-1
\end{array}\right)\right]+\sum_{i=1}^{3} \frac{n_{[3] \backslash\{i\}}}{k}\left(\begin{array}{c}
n_{i}-d-3+k \\
k-1
\end{array}\right) \\
& =\sum_{R \in X_{2}}\left[\left(\begin{array}{c}
n_{R}-d-3+k \\
k
\end{array}\right)-\sum_{i \in R}\left(\begin{array}{c}
n_{i}-d-3+k \\
k
\end{array}\right)\right]=\sum_{i=1}^{3}\left(\begin{array}{c}
n_{[3] \backslash\{i\}}-d-3+k \\
k
\end{array}\right)-2 \sum_{i=1}^{3}\left(\begin{array}{c}
n_{i}-d-3+k \\
k
\end{array}\right)
\end{aligned}
$$

Hence we have:

$$
h_{k}\left(\mathcal{K}_{[3]}\right) \leq\left(\begin{array}{c}
n_{[3]}-d-3+k \\
k
\end{array}\right)-\sum_{i=1}^{3}\left(\begin{array}{c}
n_{[3] \backslash\{i\}}-d-3+k \\
k
\end{array}\right)+\sum_{i=1}^{3}\left(\begin{array}{c}
n_{i}-d-3+k \\
k
\end{array}\right)+\sum_{i=1}^{3}\left(\begin{array}{c}
n_{[3] \backslash\{i\}}-d-3+k \\
k
\end{array}\right)
$$




$$
\begin{aligned}
& -2 \sum_{i=1}^{3}\left(\begin{array}{c}
n_{i}-d-3+k \\
k
\end{array}\right)+\sum_{i=1}^{3}\left(\begin{array}{c}
n_{i}-d-3+k \\
k
\end{array}\right)+\sum_{i=1}^{3}\left[g_{k}\left(\partial P_{i}\right)-\left(\begin{array}{c}
n_{i}-d-2+k \\
k
\end{array}\right)\right] \\
= & \left(\begin{array}{c}
n_{[3]}-d-3+k \\
k
\end{array}\right)+\sum_{i=1}^{3}\left[g_{k}\left(\partial P_{i}\right)-\left(\begin{array}{c}
n_{i}-d-2+k \\
k
\end{array}\right)\right] .
\end{aligned}
$$

Since $g_{k}\left(\partial P_{i}\right)-\left(\begin{array}{c}n_{i}-d-2+k \\ k\end{array}\right) \leq 0$, for all $k \geq 0$, we get the sought-for bound in (44) for $0 \leq k \leq d+2$. Furthermore, for $d$ odd and $k=\left\lfloor\frac{d}{2}\right\rfloor+1$, we have $g_{k}\left(\partial P_{i}\right)=0$, which yields the bound in (45).

To prove the equality claim, we distinguish between the cases $k \leq\left\lfloor\frac{d}{2}\right\rfloor$, and $k=$ $\left\lfloor\frac{d}{2}\right\rfloor+1$ with $d$ odd. Consider the case $k \leq\left\lfloor\frac{d}{2}\right\rfloor$ first, and assume that $h_{k}\left(\mathcal{K}_{[3]}\right)=\left({ }^{n_{[3]}-d-3+k} k\right.$. From relation $(75)$ we deduce that both $h_{k}\left(\mathcal{F}_{[3]}\right)$ and $g_{k}\left(\partial P_{i}\right), 1 \leq i \leq 3$, must be equal to their maximum values, since otherwise we would have that $h_{k}\left(\mathcal{K}_{[3]}\right)<\left(\begin{array}{c}n_{[3]}-d-3+k \\ k\end{array}\right)$. In view of Lemma 10, the maximality of $h_{k}\left(\mathcal{F}_{[3]}\right)$ implies that $f_{l-1}\left(\mathcal{F}_{[3]}\right)=\sum_{\emptyset \subset S \subseteq[3]}(-1)^{3-|S|}\left(\begin{array}{c}n_{S} \\ l\end{array}\right)$, for all $0 \leq l \leq k$, whereas the maximality of $g_{k}\left(\partial P_{i}\right)$ implies that $P_{i}$ is $k$-neighborly, for all $1 \leq i \leq 3$, i.e., for all $1 \leq i \leq 3, f_{l-1}\left(\partial P_{i}\right)=f_{l-1}\left(\mathcal{F}_{\{i\}}\right)=\left(\begin{array}{c}n_{i} \\ l\end{array}\right)$, for all $0 \leq l \leq k$. But then we also have that $g_{k-1}\left(\partial P_{i}\right)=\left(\begin{array}{c}n_{i}-d-2+k-1 \\ k-1\end{array}\right)$, which gives:

$$
g_{k}^{(2)}\left(\partial P_{i}\right)=g_{k}\left(\partial P_{i}\right)-g_{k-1}\left(\partial P_{i}\right)=\left(\begin{array}{c}
n_{i}-d-2+k \\
k
\end{array}\right)-\left(\begin{array}{c}
n_{i}-d-2-k-1 \\
k-1
\end{array}\right)=\left(\begin{array}{c}
n_{i}-d-3+k \\
k
\end{array}\right) .
$$

By relation (37), the maximality of $h_{k}\left(\mathcal{F}_{[3]}\right)$ implies that $g_{k-1}\left(\mathcal{F}_{[3] \backslash\{i\}}\right)$ attains its maximum value for all $1 \leq i \leq 3$. By following the argumentation in the proof of Lemma 9, the maximality of $g_{k-1}\left(\mathcal{F}_{[3] \backslash\{i\}}\right)$ further implies that $h_{l}\left(\mathcal{F}_{[3] \backslash\{i\}}\right)$ is maximal, for all $0 \leq l \leq k-1$. Solving, now, equation (13) (for $R \equiv[3])$ in terms of the sum of the $h_{k}\left(\mathcal{F}_{[3] \backslash\{i\}}\right)$ 's we get:

$$
\sum_{i=1}^{3} h_{k}\left(\mathcal{F}_{[3] \backslash\{i\}}\right)=h_{k}\left(\mathcal{K}_{[3]}\right)-h_{k}\left(\mathcal{F}_{[3]}\right)+\sum_{i=1}^{3} h_{k-1}\left(\mathcal{F}_{[3] \backslash\{i\}}\right)-\sum_{i=1}^{3} g_{k}^{(2)}\left(\partial P_{i}\right) .
$$

Substituting in the above equation the values for $h_{k}\left(\mathcal{K}_{[3]}\right), h_{k}\left(\mathcal{F}_{[3]}\right), h_{k-1}\left(\mathcal{F}_{[3] \backslash\{i\}}\right)$ and $g_{k}^{(2)}\left(\partial P_{i}\right)$, it is easy to verify that

$$
\sum_{i=1}^{3} h_{k}\left(\mathcal{F}_{[3] \backslash\{i\}}\right)=\sum_{i=1}^{3}\left[\left(\begin{array}{c}
n_{[3] \backslash\{i\}}-d-2+k \\
k
\end{array}\right)-\sum_{j \in[3] \backslash\{i\}}\left(\begin{array}{c}
n_{j}-d-2+k \\
k
\end{array}\right)\right] .
$$

In other words, the sum of the $h_{k}\left(\mathcal{F}_{[3] \backslash\{i\}}\right)$ 's attains its maximum value, which implies that each of the summands attains its maximum value. We thus conclude that $h_{l}\left(\mathcal{F}_{[3] \backslash\{i\}}\right)$ is maximal, for all $0 \leq l \leq k$, which, by [19, Lemma 3.3], implies that, for all $R \in X_{2}$, $f_{l-1}\left(\mathcal{F}_{R}\right)=\sum_{\emptyset \subset S \subseteq R}(-1)^{2-|S|}\left(\begin{array}{c}n_{S} \\ l\end{array}\right)$, for all $0 \leq l \leq k$.

Let us now consider the reverse direction and assume that for all $\emptyset \subset R \subseteq[3]$, $f_{l-1}\left(\mathcal{F}_{R}\right)=\sum_{\emptyset \subset S \subseteq R}(-1)^{|R|-|S|}\left(\begin{array}{c}n_{S} \\ l\end{array}\right)$, for all $0 \leq l \leq k$ (for $k \leq\left\lfloor\frac{d}{2}\right\rfloor, \min \left\{k,\left\lfloor\frac{d+|R|-1}{2}\right\rfloor\right\}=k$ ). Using Lemma 10, the condition above, for $R=[3]$, implies that $h_{l}\left(\mathcal{F}_{[3]}\right)$ attains its upper bound value for all $0 \leq l \leq k$. Using [19, Lemma 3.3], the condition above, for $R \in X_{2}$, implies that $h_{l}\left(\mathcal{F}_{R}\right)$ attains its upper bound value for all $0 \leq l \leq k$, and thus $g_{k}\left(\mathcal{F}_{R}\right)$ attains 
its upper bound value. Finally, the condition above, for $1 \leq i \leq 3$, implies that $P_{i}$ is $k$-neighborly, which means that $g_{l}\left(\partial P_{i}\right)=g_{l}\left(\mathcal{F}_{\{i\}}\right)=\left(\begin{array}{c}n_{i}-d-2+l \\ l\end{array}\right)$, for all $0 \leq l \leq k$, and thus (cf. (76)) $g_{k}^{(2)}\left(\partial P_{i}\right)=g_{k}^{(2)}\left(\mathcal{F}_{\{i\}}\right)=\left(\begin{array}{c}n_{i}-d-3+k \\ k\end{array}\right)$. Appealing now to relation (13) for $R \equiv[3]$, it is easy to verify that $h_{k}\left(\mathcal{K}_{[3]}\right)=\left(\begin{array}{c}n_{[3]}-d-3+k \\ k\end{array}\right)$.

We end the equality claim proof by considering the case $k=\left\lfloor\frac{d}{2}\right\rfloor+1$, for $d$ odd. Since for $d$ odd, $g_{\left\lfloor\frac{d}{2}\right\rfloor+1}\left(\partial P_{i}\right)=0$, relation $(75)$, simplifies to:

$$
h_{\left\lfloor\frac{d}{2}\right\rfloor+1}\left(\mathcal{K}_{[3]}\right) \leq h_{\left\lfloor\frac{d}{2}\right\rfloor+1}\left(\mathcal{F}_{[3]}\right)+\sum_{R \in \mathcal{X}_{2}} \frac{n_{R}-d-3}{\left\lfloor\frac{d}{2}\right\rfloor+1} h_{\left\lfloor\frac{d}{2}\right\rfloor}\left(\mathcal{F}_{R}\right)+\sum_{i=1}^{3} \frac{n_{[3] \backslash\{i\}}-\left\lfloor\frac{d}{2}\right\rfloor-1}{\left\lfloor\frac{d}{2}\right\rfloor+1} g_{\left\lfloor\frac{d}{2}\right\rfloor}\left(\partial P_{i}\right),
$$

while relation (13) (with $R \equiv[3])$ simplifies to:

$$
h_{\left\lfloor\frac{d}{2}\right\rfloor+1}\left(\mathcal{K}_{[3]}\right)=h_{\left\lfloor\frac{d}{2}\right\rfloor+1}\left(\mathcal{F}_{[3]}\right)+\sum_{i=1}^{3} g_{\left\lfloor\frac{d}{2}\right\rfloor+1}\left(\mathcal{F}_{[3] \backslash\{i\}}\right)-\sum_{i=1}^{3} g_{\left\lfloor\frac{d}{2}\right\rfloor}\left(\partial P_{i}\right) .
$$

The argument in this case is essentially the same as before. Assuming that $h_{\left\lfloor\frac{d}{2}\right\rfloor+1}\left(\mathcal{K}_{[3]}\right)$ is maximal, we deduce, from $(77)$, that both $h_{\left\lfloor\frac{d}{2}\right\rfloor+1}\left(\mathcal{F}_{[3]}\right)$ and $g_{\left\lfloor\frac{d}{2}\right\rfloor}\left(\partial P_{i}\right)$ are maximal, which, imply, respectively, that $f_{l-1}\left(\mathcal{F}_{[3]}\right)=\sum_{\emptyset \subset S \subseteq[3]}(-1)^{3-|S|}\left(\begin{array}{c}n_{S} \\ l\end{array}\right)$, for all $0 \leq l \leq\left\lfloor\frac{d}{2}\right\rfloor+1=\left\lfloor\frac{d+2}{2}\right\rfloor$, and that, for all $1 \leq i \leq 3, f_{l-1}\left(\mathcal{F}_{\{i\}}\right)=\left(\begin{array}{c}n_{i} \\ l\end{array}\right)$, for all $0 \leq l \leq\left\lfloor\frac{d}{2}\right\rfloor$. The maximality of $h_{\left\lfloor\frac{d}{2}\right\rfloor+1}\left(\mathcal{F}_{[3]}\right)$ implies also the maximality of $g_{l}\left(\mathcal{F}_{R}\right)$, for all $R \in \mathcal{X}_{2}$, and for all $0 \leq l \leq\left\lfloor\frac{d}{2}\right\rfloor$, and thus the maximality of $h_{l}\left(\mathcal{F}_{R}\right)$, for all $R \in X_{2}$, and for all $0 \leq l \leq\left\lfloor\frac{d}{2}\right\rfloor$. By solving equation (78) in terms of the sum of the $h_{\left\lfloor\frac{d}{2}\right\rfloor+1}\left(\mathcal{F}_{R}\right)^{\text {'s, }}$, we also deduce that $h_{\left\lfloor\frac{d}{2}\right\rfloor+1}\left(\mathcal{F}_{R}\right)$ is maximal, for all $R \in X_{2}$. Hence, we have that $h_{l}\left(\mathcal{F}_{R}\right)$ is maximal, for all $R \in X_{2}$, and for all $0 \leq l \leq\left\lfloor\frac{d}{2}\right\rfloor+1=\left\lfloor\frac{d+1}{2}\right\rfloor$, which, by [19, Lemma 3.3], gives that $f_{l-1}\left(\mathcal{F}_{R}\right)=$ $\sum_{\emptyset \subset S \subseteq R}(-1)^{2-|S|}\left(\begin{array}{c}n_{S} \\ l\end{array}\right)$, for all $R \in \mathcal{X}_{2}$, and for all $0 \leq l \leq\left\lfloor\frac{d+1}{2}\right\rfloor$.

Assuming now that, for all $\emptyset \subset R \subseteq[3], f_{l-1}\left(\mathcal{F}_{R}\right)=\sum_{\emptyset \subset S \subseteq R}(-1)^{|R|-|S|}\left(\begin{array}{c}n_{S} \\ l\end{array}\right)$, for all $0 \leq$ $l \leq \min \left\{\left\lfloor\frac{d}{2}\right\rfloor+1,\left\lfloor\frac{d+|R|-1}{2}\right\rfloor\right\}$, we deduce, from Lemma 10, that that $h_{\left\lfloor\frac{d}{2}\right\rfloor+1}\left(\mathcal{F}_{[3]}\right)$ attains its upper bound value for all $0 \leq l \leq\left\lfloor\frac{d+2}{2}\right\rfloor=\left\lfloor\frac{d}{2}\right\rfloor+1$. Furthermore, Lemma 3.3 in [19], implies that, for all $R \in X_{2}, h_{l}\left(\mathcal{F}_{R}\right)$ attains its upper bound value for all $0 \leq l \leq\left\lfloor\frac{d+1}{2}\right\rfloor=\left\lfloor\frac{d}{2}\right\rfloor+1$, which means that $g_{\left\lfloor\frac{d+1}{2}\right\rfloor}\left(\mathcal{F}_{R}\right)$ attains its upper bound value, for all $R \in X_{2}$. Finally, our assumption above, implies that, for all $1 \leq i \leq 3, P_{i}$ is neighborly, which means that $g_{\left\lfloor\frac{d}{2}\right\rfloor}\left(\partial P_{i}\right)=\left(\begin{array}{c}n_{i}-\left\lfloor\frac{d}{2}\right\rfloor-3 \\ \left\lfloor\frac{d}{2}\right\rfloor\end{array}\right)$. Appealing to relation (78) above, it is easy to verify that $h_{\left\lfloor\frac{d}{2}\right\rfloor+1}\left(\mathcal{K}_{[3]}\right)$ attains its upper bound in $(45)$.

\section{B Asymptotic analysis of Vandermonde-like determinants}

We start by introducing what is known as Laplace's Expansion Theorem for determinants (see $[11,15]$ for details and proofs). Consider a $n \times n$ matrix $A$. Let $\boldsymbol{r}=\left(r_{1}, r_{2}, \ldots, r_{k}\right)$, be a vector of $k$ row indices for $A$, where $1 \leq k<n$ and $1 \leq r_{1}<r_{2}<\ldots<r_{k} \leq n$. Let $\boldsymbol{c}=\left(c_{1}, c_{2}, \ldots, c_{k}\right)$ be a vector of $k$ column indices for $A$, where $1 \leq k<n$ and 
$1 \leq c_{1}<c_{2}<\ldots<c_{k} \leq n$. We denote by $S(A ; \boldsymbol{r}, \boldsymbol{c})$ the $k \times k$ submatrix of $A$ constructed by keeping the entries of $A$ that belong to a row in $\boldsymbol{r}$ and a column in $\boldsymbol{c}$. The complementary submatrix for $S(A ; \boldsymbol{r}, \boldsymbol{c})$, denoted by $\bar{S}(A ; \boldsymbol{r}, \boldsymbol{c})$, is the $(n-k) \times(n-k)$ submatrix of $A$ constructed by removing the rows and columns of $A$ in $\boldsymbol{r}$ and $\boldsymbol{c}$, respectively. Then, the determinant of $A$ can be computed by expanding in terms of the $k$ columns of $A$ in $\boldsymbol{c}$ according to the following theorem.

Theorem 15 (Laplace's Expansion Theorem). Let $A$ be a $n \times n$ matrix. Let $\boldsymbol{c}=$ $\left(c_{1}, c_{2}, \ldots, c_{k}\right)$ be a vector of $k$ column indices for $A$, where $1 \leq k<n$ and $1 \leq c_{1}<c_{2}<$ $\ldots<c_{k} \leq n$. Then:

$$
\operatorname{det}(A)=\sum_{\boldsymbol{r}}(-1)^{|\boldsymbol{r}|+|\boldsymbol{c}|} \operatorname{det}(S(A ; \boldsymbol{r}, \boldsymbol{c})) \operatorname{det}(\bar{S}(A ; \boldsymbol{r}, \boldsymbol{c}))
$$

where $|\boldsymbol{r}|=r_{1}+r_{2}+\ldots+r_{k},|\boldsymbol{c}|=c_{1}+c_{2}+\ldots+c_{k}$, and the summation is taken over all row vectors $\boldsymbol{r}=\left(r_{1}, r_{2}, \ldots, r_{k}\right)$ of $k$ row indices for $A$, where $1 \leq r_{1}<r_{2}<\ldots<r_{k} \leq n$.

In what follows we recall some facts concerning generalized Vandermonde determinants that will be in use to us later. Let $n \geq 2, \boldsymbol{x}=\left(x_{1}, \ldots, x_{n}\right)$ and $\boldsymbol{\mu}=\left(\mu_{1}, \mu_{2}, \ldots, \mu_{n}\right)$, where we require that $0 \leq \mu_{1}<\mu_{2}<\ldots<\mu_{n}$. The generalized Vandermonde determinant, denoted by $\operatorname{GVD}(\boldsymbol{x} ; \boldsymbol{\mu})$, is the $n \times n$ determinant whose $i$ th row is the vector $\boldsymbol{x}$ with all its entries raised to $\mu_{i}$. While there is no general formula for the generalized Vandermonde determinant, it is a well-known fact that, if the elements of $\boldsymbol{x}$ are in strictly increasing order, then $\operatorname{GVD}(\boldsymbol{x} ; \boldsymbol{\mu})>0$ (for example, see [12] for a proof of this fact).

In the remainder of this section we consider two determinants that are parameterized by a positive parameter $\tau$, and we study their asymptotic behavior with respect to $\tau$. These determinants are generalizations of the determinants that arise in the proofs of Lemmas 13 and 14 in Section 6, and are directly associated with the equations of some appropriately defined supporting hyperplanes for the faces of $\mathcal{F}_{R}$ where $R \in \mathcal{X}_{2}$ or $R \equiv$ [3] (recall that $\mathcal{F}_{R}$ stands for the set of faces of the Cayley polytope of $|R|$ polytopes $P_{i}, i \in R$, with the property that each face in $\mathcal{F}_{R}$ has at least one vertex from each polytope $P_{i}$ ). The two determinants that we study are generalized-Vandermonde-like determinants that are polynomial functions of $\tau$, and correspond, respectively, to the two cases $R \in X_{2}$ and $R \equiv[3]$ mentioned above. Since in Section 6 we are interested in small values of $\tau$, our asymptotic analysis in the two lemmas below is targeted towards revealing the term of $\tau$ of minimal exponent.

We start-off with the generalized version of the determinant that arises in the upper bound tightness construction in Section 6 when $R \in X_{2}$.

Lemma 16. Fix two integers $m \geq 2$ and $n \geq 2$, with $n+m \geq 5$. Let $D_{n, m}(\tau ; I, J, \boldsymbol{\mu})$ be 
the $(n+m) \times(n+m)$ determinant:

$$
(-1)^{J+1}\left|\begin{array}{cccccc}
\left(x_{1} \tau^{\alpha}\right)^{\mu_{1}} & \cdots & \left(x_{n} \tau^{\alpha}\right)^{\mu_{1}} & 0 & \cdots & 0 \\
0 & \cdots & 0 & \left(y_{1} \tau^{\beta}\right)^{\mu_{2}} & \cdots & \left(y_{m} \tau^{\beta}\right)^{\mu_{2}} \\
f_{3}(\tau)\left(x_{1} \tau^{\alpha}\right)^{\mu_{3}} & \cdots & f_{3}(\tau)\left(x_{n} \tau^{\alpha}\right)^{\mu_{3}} & g_{3}(\tau)\left(y_{1} \tau^{\beta}\right)^{\mu_{3}} & \cdots & g_{3}(\tau)\left(y_{m} \tau^{\beta}\right)^{\mu_{3}} \\
f_{4}(\tau)\left(x_{1} \tau^{\alpha}\right)^{\mu_{4}} & \cdots & f_{4}(\tau)\left(x_{n} \tau^{\alpha}\right)^{\mu_{4}} & g_{4}(\tau)\left(y_{1} \tau^{\beta}\right)^{\mu_{4}} & \cdots & g_{4}(\tau)\left(y_{m} \tau^{\beta}\right)^{\mu_{4}} \\
f_{5}(\tau)\left(x_{1} \tau^{\alpha}\right)^{\mu_{5}} & \cdots & f_{5}(\tau)\left(x_{n} \tau^{\alpha}\right)^{\mu_{5}} & g_{5}(\tau)\left(y_{1} \tau^{\beta}\right)^{\mu_{5}} & \cdots & g_{5}(\tau)\left(y_{m} \tau^{\beta}\right)^{\mu_{3}} \\
\left(x_{1} \tau^{\alpha}\right)^{\mu_{6}} & \cdots & \left(x_{n} \tau^{\alpha}\right)^{\mu_{6}} & \left(y_{1} \tau^{\beta}\right)^{\mu_{6}} & \cdots & \left(y_{m} \tau^{\beta}\right)^{\mu_{6}} \\
\left(x_{1} \tau^{\alpha}\right)^{\mu_{7}} & \cdots & \left(x_{n} \tau^{\alpha}\right)^{\mu_{7}} & \left(y_{1} \tau^{\beta}\right)^{\mu_{7}} & \cdots & \left(y_{m} \tau^{\beta}\right)^{\mu_{7}} \\
\vdots & \ddots & \vdots & \vdots & \ddots & \vdots \\
\left(x_{1} \tau^{\alpha}\right)^{\mu_{\ell}} & \cdots & \left(x_{n} \tau^{\alpha}\right)^{\mu_{\ell}} & \left(y_{1} \tau^{\beta}\right)^{\mu_{\ell}} & \cdots & \left(y_{m} \tau^{\beta}\right)^{\mu_{\ell}}
\end{array}\right|
$$

where $0<x_{1}<x_{2}<\ldots<x_{n}, 0<y_{1}<y_{2}<\ldots<y_{m}, \ell=n+m, \boldsymbol{\mu}=\left(\mu_{1}, \ldots, \mu_{\ell}\right)$, with $0 \leq \mu_{1} \leq \mu_{2}<\mu_{3}<\ldots<\mu_{\ell},(I, J) \in\{(3,4),(3,5),(4,5)\}, f_{I}(\tau)=g_{J}(\tau)=1$, $f_{i}(\tau)=g_{j}(\tau)=\tau^{M}$, for $i \neq I$ and $j \neq J, \alpha>\beta \geq 0, M \geq \alpha|\boldsymbol{\mu}|$ and $\tau>0$. Then:

$$
\begin{aligned}
& D_{n, m}(\tau ; I, J, \boldsymbol{\mu})=C \tau^{\xi}+\Theta\left(\tau^{\xi+1}\right), \\
& \xi=\alpha\left(\mu_{1}+\mu_{3}+\sum_{i=4}^{n+2} \mu_{i}-\mu_{J}\right)+\beta\left(\mu_{2}+\mu_{J}+\sum_{i=n+3}^{\ell} \mu_{i}\right),
\end{aligned}
$$

where $C$ is a positive constant independent of $\tau$.

Proof. For simplicity, we write $D_{n, m}(\tau)$ instead of $D_{n, m}(\tau ; I, J, \boldsymbol{\mu})$, suppressing $I, J$ and $\boldsymbol{\mu}$ in the notation. We denote by $\Delta_{n, m}(\tau)$ the matrix corresponding to the determinant $(-1)^{J+1} D_{n, m}(\tau)$. If we apply Laplace's expansion with respect to the first $n$ columns, i.e., when $\boldsymbol{c}=(1,2, \ldots, n)$, we get:

$$
\begin{aligned}
D_{n, m}(\tau) & =(-1)^{J+1} \sum_{\substack{\boldsymbol{r}=\left(r_{1}, r_{2}, \ldots, r_{n}\right) \\
1 \leq r_{1}<r_{2}<\cdots<r_{n} \leq n+m}}(-1)^{|\boldsymbol{r}|+|\boldsymbol{c}|} \operatorname{det}\left(S\left(\Delta_{n, m}(\tau) ; \boldsymbol{r}, \boldsymbol{c}\right)\right) \operatorname{det}\left(\bar{S}\left(\Delta_{n, m}(\tau) ; \boldsymbol{r}, \boldsymbol{c}\right)\right) \\
& =\sum_{\substack{\boldsymbol{r}=\left(r_{1}, r_{2}, \ldots, r_{n}\right) \\
1 \leq r_{1}<r_{2}<\cdots<r_{n} \leq n+m}}(-1)^{|\boldsymbol{r}|+\frac{n(n+1)}{2}+J+1} \operatorname{det}\left(S\left(\Delta_{n, m}(\tau) ; \boldsymbol{r}, \boldsymbol{c}\right)\right) \operatorname{det}\left(\bar{S}\left(\Delta_{n, m}(\tau) ; \boldsymbol{r}, \boldsymbol{c}\right)\right) .
\end{aligned}
$$

The above sum consists of $\left(\begin{array}{c}n+m \\ n\end{array}\right)$ terms. Among these terms:

(i) all those for which $\boldsymbol{r}$ contains the second row vanish (in this case the corresponding row of $S\left(\Delta_{n, m}(\tau) ; \boldsymbol{r}, \boldsymbol{c}\right)$ consists of zeros), and

(ii) all those for which $\boldsymbol{r}$ does not contain the first row vanish (in this case at least one row of $\bar{S}\left(\Delta_{n, m}(\tau) ; \boldsymbol{r}, \boldsymbol{c}\right)$ consists of zeros). 
The remaining terms of the expansion are the $\left(\begin{array}{c}n+m-2 \\ n-1\end{array}\right)$ terms for which $\boldsymbol{r}$ contains 1 but not 2, i.e., $\boldsymbol{r}=\left(1, r_{2}, r_{3}, \ldots, r_{n}\right)$, with $3 \leq r_{2}<r_{3}<\ldots<r_{n} \leq n+m$. For any given $\boldsymbol{r}$, we denote by $\overline{\boldsymbol{r}}$ the vector of the $m$, among the $n+m$, row indices for $\Delta_{n, m}(\tau)$ that do not belong to $\boldsymbol{r}$ (recall that 2 always belongs to $\overline{\boldsymbol{r}}$ ). Notice that the elements of the $k$ th row of $\Delta_{n, m}(\tau)$ have exponent $\mu_{k}$. Denoting by $\boldsymbol{\mu}_{\boldsymbol{r}}$ the vector the $i$ th element of which is $\mu_{r_{i}}$, we have that:

(i) $\operatorname{det}\left(S\left(\Delta_{n, m}(\tau) ; \boldsymbol{r}, \boldsymbol{c}\right)\right)$ is the $n \times n$ generalized Vandermonde determinant $\operatorname{GVD}\left(\tau^{\alpha} \boldsymbol{x}\right.$; $\left.\boldsymbol{\mu}_{\boldsymbol{r}}\right)$, multiplied by $\tau^{M}$ if $J \in \boldsymbol{r}$.

(ii) $\operatorname{det}\left(\bar{S}\left(\Delta_{n, m}(\tau) ; \boldsymbol{r}, \boldsymbol{c}\right)\right)$ is the $m \times m$ generalized Vandermonde determinant $\operatorname{GVD}\left(\tau^{\beta} \boldsymbol{y}\right.$; $\left.\boldsymbol{\mu}_{\overline{\boldsymbol{r}}}\right)$, multiplied by $\tau^{M}$ if $I \in \overline{\boldsymbol{r}}$.

We can, thus, simplify the expansion in (80) to get:

$$
\begin{aligned}
D_{n, m}(\tau) & =\sum_{\{\boldsymbol{r} \mid 1 \in \boldsymbol{r}, 2 \notin \boldsymbol{r}\}}(-1)^{|\boldsymbol{r}|+\frac{n(n+1)}{2}+J+1} h(\boldsymbol{r}, \tau ; I, J) \operatorname{GVD}\left(\tau^{\alpha} \boldsymbol{x} ; \boldsymbol{\mu}_{\boldsymbol{r}}\right) \operatorname{GVD}\left(\tau^{\beta} \boldsymbol{y} ; \boldsymbol{\mu}_{\overline{\boldsymbol{r}}}\right) \\
& =\sum_{\{\boldsymbol{r} \mid 1 \in \boldsymbol{r}, 2 \notin \boldsymbol{r}\}}(-1)^{|\boldsymbol{r}|+\frac{n(n+1)}{2}+J+1} h(\boldsymbol{r}, \tau ; I, J) \tau^{\alpha\left|\boldsymbol{\mu}_{\boldsymbol{r}}\right|+\beta\left|\boldsymbol{\mu}_{\overline{\boldsymbol{r}} \mid}\right|} \operatorname{GVD}\left(\boldsymbol{x} ; \boldsymbol{\mu}_{\boldsymbol{r}}\right) \operatorname{GVD}\left(\boldsymbol{y} ; \boldsymbol{\mu}_{\overline{\boldsymbol{r}}}\right),
\end{aligned}
$$

where

$$
h(\boldsymbol{r}, \tau ; I, J)= \begin{cases}1, & I \in \boldsymbol{r} \text { and } J \notin \boldsymbol{r} \\ \tau^{2 M}, & I \notin \boldsymbol{r} \text { and } J \in \boldsymbol{r} \\ \tau^{M}, & \text { otherwise. }\end{cases}
$$

In the remainder of the proof we seek to find the unique term in the expansion (81) that corresponds to the minimum order of $\tau$, or, equivalently, the minimum exponent for $\tau$. Since $\alpha>\beta \geq 0$, for any $\boldsymbol{r}$, with $I \in \boldsymbol{r}$ and $J \notin \boldsymbol{r}$, the exponent of $\tau$ is:

$$
\alpha\left|\boldsymbol{\mu}_{\boldsymbol{r}}\right|+\beta\left|\boldsymbol{\mu}_{\bar{r}}\right|<\alpha\left|\boldsymbol{\mu}_{\boldsymbol{r}}\right|+\alpha\left|\boldsymbol{\mu}_{\bar{r}}\right|=\alpha|\boldsymbol{\mu}| \leq M,
$$

where we used the fact that:

$$
\left|\boldsymbol{\mu}_{\boldsymbol{r}}\right|+\left|\boldsymbol{\mu}_{\overline{\boldsymbol{r}}}\right|=\sum_{i=1}^{n} \mu_{r_{i}}+\sum_{i=1}^{m} \mu_{\bar{r}_{i}}=\sum_{i \in \boldsymbol{r}} \mu_{i}+\sum_{i \in \overline{\boldsymbol{r}}} \mu_{i}=\sum_{i=1}^{\ell} \mu_{i}=|\boldsymbol{\mu}| .
$$

This implies that the terms in (81) that correspond to the row vectors $\boldsymbol{r}$ that contain $J$ cannot be the terms of minimal order of $\tau$, since for these terms the exponent of $\tau$ is at least

$$
\alpha\left|\boldsymbol{\mu}_{\boldsymbol{r}}\right|+\beta\left|\boldsymbol{\mu}_{\overline{\boldsymbol{r}}}\right|+M>\beta\left|\boldsymbol{\mu}_{\boldsymbol{r}}\right|+\beta\left|\boldsymbol{\mu}_{\overline{\boldsymbol{r}}}\right|+M=\beta|\boldsymbol{\mu}|+M \geq M .
$$

For the remaining terms, i.e., for those $\boldsymbol{r}$ that do not contain $J$, we have $h(\boldsymbol{r}, \tau$; $I, J)=1$. For these terms the exponent of $\tau$ is $\alpha\left|\boldsymbol{\mu}_{\boldsymbol{r}}\right|+\beta\left|\boldsymbol{\mu}_{\overline{\boldsymbol{r}}}\right|$. Since $\alpha>\beta$, we may write $\alpha=\beta+\theta$ for some $\theta>0$. This gives:

$$
\alpha\left|\boldsymbol{\mu}_{\boldsymbol{r}}\right|+\beta\left|\boldsymbol{\mu}_{\overline{\boldsymbol{r}}}\right|=(\beta+\theta)\left|\boldsymbol{\mu}_{\boldsymbol{r}}\right|+\beta\left|\boldsymbol{\mu}_{\overline{\boldsymbol{r}}}\right|=\beta|\boldsymbol{\mu}|+\theta\left|\boldsymbol{\mu}_{\boldsymbol{r}}\right| .
$$

Clearly, in this case, the quantity $\alpha\left|\boldsymbol{\mu}_{\boldsymbol{r}}\right|+\beta\left|\boldsymbol{\mu}_{\overline{\boldsymbol{r}}}\right|$ attains its minimum when $\left|\boldsymbol{\mu}_{\boldsymbol{r}}\right|$ is minimal. We distinguish between the following cases: 
- $(I, J)=(3,4)$. In this case $\left|\boldsymbol{\mu}_{\boldsymbol{r}}\right|$ attains its minimal value if and only if $\boldsymbol{r}$ is equal to $\boldsymbol{\rho}=(1,3,5,6, \ldots, n+2)$. Furthermore,

$$
\begin{aligned}
& \left|\boldsymbol{\mu}_{\boldsymbol{\rho}}\right|=\mu_{1}+\mu_{3}+\sum_{i=5}^{n+2} \mu_{i}=\mu_{1}+\mu_{3}+\sum_{i=4}^{n+2} \mu_{i}-\mu_{J}, \\
& \left|\boldsymbol{\mu}_{\overline{\boldsymbol{\rho}}}\right|=\mu_{2}+\mu_{4}+\sum_{i=n+3}^{\ell} \mu_{i}=\mu_{2}+\mu_{J}+\sum_{i=n+3}^{\ell} \mu_{i}
\end{aligned}
$$

and

$$
\begin{aligned}
|\boldsymbol{\rho}|+\frac{n(n+1)}{2}+J+1 & =\sum_{i=1}^{n+2} i-(2+4)+\frac{n(n+1)}{2}+4+1 \\
& =\frac{(n+2)(n+3)}{2}+\frac{n(n+1)}{2}-1 \\
& =n^{2}+3 n+3-1 \\
& =(n+1)(n+2),
\end{aligned}
$$

which is even for any $n \geq 2$.

- $I \in\{3,4\}$ and $J=5$. In this case $\left|\boldsymbol{\mu}_{\boldsymbol{r}}\right|$ attains its minimal value if and only if $\boldsymbol{r}$ is equal to $\boldsymbol{\rho}=(1,3,4,6, \ldots, n+2)$. Furthermore,

$$
\begin{aligned}
& \left|\boldsymbol{\mu}_{\boldsymbol{\rho}}\right|=\mu_{1}+\mu_{3}+\mu_{4}+\sum_{i=6}^{n+2}=\mu_{1}+\mu_{3}+\sum_{i=4}^{n+2}-\mu_{J}, \\
& \left|\boldsymbol{\mu}_{\overline{\boldsymbol{\rho}}}\right|=\mu_{2}+\mu_{5}+\sum_{i=n+3}^{\ell}=\mu_{2}+\mu_{J}+\sum_{i=n+3}^{\ell} \mu_{i},
\end{aligned}
$$

and

$$
\begin{aligned}
|\boldsymbol{\rho}|+\frac{n(n+1)}{2}+J+1 & =\sum_{i=1}^{n+1} i-(2+5)+\frac{n(n+1)}{2}+5+1 \\
& =\frac{(n+2)(n+3)}{2}+\frac{n(n+1)}{2}-1 \\
& =n^{2}+3 n+3-1 \\
& =(n+1)(n+2),
\end{aligned}
$$

which is again even for any $n \geq 2$.

We can thus rewrite (81) in the following form:

$$
D_{n, m}(\tau)=\tau^{\alpha\left|\boldsymbol{\mu}_{\boldsymbol{\rho}}\right|+\beta\left|\boldsymbol{\mu}_{\overline{\boldsymbol{\rho}}}\right|} \operatorname{GVD}\left(\boldsymbol{x} ; \boldsymbol{\mu}_{\boldsymbol{\rho}}\right) \operatorname{GVD}\left(\boldsymbol{y} ; \boldsymbol{\mu}_{\overline{\boldsymbol{\rho}}}\right)+\Theta\left(\tau^{\alpha\left|\boldsymbol{\mu}_{\boldsymbol{\rho}}\right|+\beta\left|\boldsymbol{\mu}_{\overline{\boldsymbol{\rho}}}\right|+1}\right) .
$$

The lemma immediately follows from the positivity of the generalized Vandermonde determinants $\operatorname{GVD}\left(\boldsymbol{x} ; \boldsymbol{\mu}_{\boldsymbol{\rho}}\right)$ and $\operatorname{GVD}\left(\boldsymbol{y} ; \boldsymbol{\mu}_{\overline{\boldsymbol{\rho}}}\right)$. 
We end with the following lemma, where we perform the asymptotic analysis of the generalized version of the determinant that arises in the upper bound tightness construction in Section 6 when $R \equiv[3]$.

Lemma 17. Fix three integers $m \geq 2, n \geq 2$ and $k \geq 2$, with $n+m+k \geq 7$. Let $E_{n, m, k}(\tau ; \boldsymbol{\mu})$ be the $(n+m+k) \times(n+m+k)$ determinant:

$$
-\left|\begin{array}{ccccccccc}
\left(x_{1} \tau^{2}\right)^{\mu_{1}} & \cdots & \left(x_{n} \tau^{2}\right)^{\mu_{1}} & 0 & \cdots & 0 & 0 & \cdots & 0 \\
0 & \cdots & 0 & \left(y_{1} \tau\right)^{\mu_{2}} & \cdots & \left(y_{m} \tau\right)^{\mu_{2}} & 0 & \cdots & 0 \\
0 & \cdots & 0 & 0 & \cdots & 0 & z_{1}^{\mu_{3}} & \cdots & z_{k}^{\mu_{3}} \\
\left(x_{1} \tau^{2}\right)^{\mu_{4}} & \cdots & \left(x_{n} \tau^{2}\right)^{\mu_{4}} & \tau^{M}\left(y_{1} \tau\right)^{\mu_{4}} & \cdots & \tau^{M}\left(y_{n} \tau\right)^{\mu_{4}} & \tau^{M} z_{1}^{\mu_{4}} & \cdots & \tau^{M} z_{n}^{\mu_{4}} \\
\tau^{M}\left(x_{1} \tau^{2}\right)^{\mu_{5}} & \cdots & \tau^{M}\left(x_{n} \tau^{2}\right)^{\mu_{5}} & \left(y_{1} \tau\right)^{\mu_{5}} & \cdots & \left(y_{m} \tau\right)^{\mu_{5}} & \tau^{M} z_{1}^{\mu_{5}} & \cdots & \tau^{M} z_{n}^{\mu_{5}} \\
\tau^{M}\left(x_{1} \tau^{2}\right)^{\mu_{6}} & \cdots & \tau^{M}\left(x_{n} \tau^{2}\right)^{\mu_{6}} & \tau^{M}\left(y_{1} \tau\right)^{\mu_{6}} & \cdots & \tau^{M}\left(y_{m} \tau\right)^{\mu_{6}} & z_{1}^{\mu_{6}} & \cdots & z_{m}^{\mu_{6}} \\
\left(x_{1} \tau^{2}\right)^{\mu_{7}} & \cdots & \left(x_{n} \tau^{2}\right)^{\mu_{7}} & \left(y_{1} \tau\right)^{\mu_{7}} & \cdots & \left(y_{m} \tau\right)^{\mu_{7}} & z_{1}^{\mu_{7}} & \cdots & z_{k}^{\mu_{7}} \\
\vdots & \ddots & \vdots & \vdots & \ddots & \vdots & \vdots & \ddots & \vdots \\
\left(x_{1} \tau^{2}\right)^{\mu_{\ell}} & \cdots & \left(x_{n} \tau^{2}\right)^{\mu_{\ell}} & \left(y_{1} \tau\right)^{\mu_{\ell}} & \cdots & \left(y_{m} \tau\right)^{\mu_{\ell}} & z_{1}^{\mu_{\ell}} & \cdots & z_{k}^{\mu_{\ell}}
\end{array}\right|
$$

where $0<x_{1}<x_{2}<\ldots<x_{n}, 0<y_{1}<y_{2}<\ldots<y_{m}, 0<z_{1}<z_{2}<\ldots<z_{k}$, $\ell=n+m+k, \boldsymbol{\mu}=\left(\mu_{1}, \mu_{2}, \ldots, \mu_{\ell}\right)$, with $0 \leq \mu_{1} \leq \mu_{2} \leq \mu_{3}<\mu_{4}<\mu_{5}<\ldots<\mu_{\ell}$, $M \geq 2|\boldsymbol{\mu}|$ and $\tau>0$. Then,

$$
E_{n, m, k}(\tau ; \boldsymbol{\mu})=C^{\prime} \tau^{\xi}+\Theta\left(\tau^{\xi+1}\right), \quad \xi=2\left(\mu_{1}+\mu_{4}+\sum_{i=7}^{n+4} \mu_{i}\right)+\mu_{2}+\mu_{5}+\sum_{i=n+5}^{n+m+2} \mu_{i}
$$

where $C^{\prime}$ is a positive constant independent of $\tau$.

Proof. We write $E_{n, m, k}(\tau)$ instead of $E_{n, m, k}(\tau ; \boldsymbol{\mu})$, suppressing $\boldsymbol{\mu}$ in the notation. We denote by $\mathcal{E}_{n, m, k}(\tau)$ the matrix corresponding to the determinant $-E_{n, m, k}(\tau)$. If we apply Laplace's expansion theorem with respect to the first $n$ columns, i.e., when $\boldsymbol{c}=(1,2, \ldots, n)$, we get:

$$
\begin{aligned}
E_{n, m, k}(\tau) & =-\sum_{\boldsymbol{r}}(-1)^{|\boldsymbol{r}|+|\boldsymbol{c}|} \operatorname{det}\left(S\left(\mathcal{E}_{n, m, k}(\tau) ; \boldsymbol{r}, \boldsymbol{c}\right)\right) \operatorname{det}\left(\bar{S}\left(\mathcal{E}_{n, m, k}(\tau) ; \boldsymbol{r}, \boldsymbol{c}\right)\right) \\
& =\sum_{\boldsymbol{r}}(-1)^{|\boldsymbol{r}|+\frac{n(n+1)}{2}+1} \operatorname{det}\left(S\left(\mathcal{E}_{n, m, k}(\tau) ; \boldsymbol{r}, \boldsymbol{c}\right)\right) \operatorname{det}\left(\bar{S}\left(\mathcal{E}_{n, m, k}(\tau) ; \boldsymbol{r}, \boldsymbol{c}\right)\right) .
\end{aligned}
$$

The above sum consists of $\left(\begin{array}{c}n+m+k \\ n\end{array}\right)$ terms. Among these terms:

(i) all those for which $\boldsymbol{r}$ contains the second or third row vanish (the corresponding row of $S\left(\mathcal{E}_{n, m, k}(\tau) ; \boldsymbol{r}, \boldsymbol{c}\right)$ consists of zeros), and

(ii) all those for which $\boldsymbol{r}$ does not contain the first row vanish (in this case there exists a row of $\bar{S}\left(\mathcal{E}_{n, m, k}(\tau) ; \boldsymbol{r}, \boldsymbol{c}\right)$ that consists of zeros). 
The remaining terms of the expansion are the $\left(\begin{array}{c}n+m+k-3 \\ n-1\end{array}\right)$ terms for which $\boldsymbol{r}=\left(1, r_{2}, r_{3}, \ldots\right.$, $r_{n}$ ), with $4 \leq r_{2}<r_{3}<\ldots<r_{n} \leq n+m+k$. As a result, the expansion in (82) simplifies to:

$$
E_{n, m, k}(\tau)=\sum_{\boldsymbol{r} \in \mathcal{R}}(-1)^{|\boldsymbol{r}|+\frac{n(n+1)}{2}+1} \operatorname{det}\left(S\left(\mathcal{E}_{n, m, k}(\tau) ; \boldsymbol{r}, \boldsymbol{c}\right)\right) \operatorname{det}\left(\bar{S}\left(\mathcal{E}_{n, m, k}(\tau) ; \boldsymbol{r}, \boldsymbol{c}\right)\right) .
$$

where $\mathcal{R}$ is the set of row vectors that contain 1 , but not 2 and 3 , i.e., $\mathcal{R}=\{\boldsymbol{r} \mid 1 \in \boldsymbol{r}, 2,3 \notin$ $\boldsymbol{r}\}$.

For any given $\boldsymbol{r}$, we denote by $\overline{\boldsymbol{r}}$ the vector of the $m+k$ row indices for $\mathcal{E}_{n, m, k}(\tau)$ that do not belong to $\boldsymbol{r}$. Moreover, $\boldsymbol{\mu}_{\boldsymbol{r}}$ is the vector the $i$ th element of which is $\mu_{r_{i}}$. As in the proof of Lemma 16, we seek to find the unique minimum term in the expansion (83) that corresponds to the minimum order of $\tau$, or, equivalently, the minimum exponent for $\tau$.

Let us denote by $\mathcal{R}^{\prime}$ the set of row vectors $\mathcal{R}^{\prime}=\{\boldsymbol{r} \in \mathcal{R} \mid 4 \in \boldsymbol{r}$ and $5,6 \notin \boldsymbol{r}\}$, and let $\mathcal{R}^{\prime \prime}=\mathcal{R} \backslash \mathcal{R}^{\prime}$. For any $\boldsymbol{r} \in \mathcal{R}^{\prime}$, observe that:

(i) $\operatorname{det}\left(S\left(\mathcal{E}_{n, m, k}(\tau) ; \boldsymbol{r}, \boldsymbol{c}\right)\right)$ is the $n \times n$ generalized Vandermonde determinant $\operatorname{GVD}\left(\tau^{2} \boldsymbol{x}\right.$; $\left.\mu_{r}\right)$.

(ii) $\operatorname{det}\left(\bar{S}\left(\mathcal{E}_{n, m, k}(\tau) ; \boldsymbol{r}, \boldsymbol{c}\right)\right)$ is the $(m+k) \times(m+k)$ determinant $D_{m, k}\left(\tau ; 3,4, \boldsymbol{\mu}_{\overline{\boldsymbol{r}}}\right)$ of Lemma 16 multiplied by $(-1)^{4+1}=-1$, with $\boldsymbol{x} \leftarrow \boldsymbol{y}, \boldsymbol{y} \leftarrow \boldsymbol{z}, \boldsymbol{\mu} \leftarrow \boldsymbol{\mu}_{\bar{r}},(I, J)=(3,4), \alpha \leftarrow 1$, $\beta \leftarrow 0$ and $M \leftarrow M$ (since $M \geq 2|\boldsymbol{\mu}|>\left|\boldsymbol{\mu}_{\bar{r}}\right|$, the condition for $M$ in Lemma 16 is satisfied).

We can, thus, rewrite the expansion in (83) to get:

$$
\begin{aligned}
E_{n, m, k}(\tau)= & \sum_{\boldsymbol{r} \in \mathcal{R}^{\prime}}(-1)^{|\boldsymbol{r}|+\frac{n(n+1)}{2}+1} \operatorname{GVD}\left(\tau^{2} \boldsymbol{x} ; \boldsymbol{\mu}_{\boldsymbol{r}}\right)\left(-D_{m, k}\left(\tau ; 3,4, \boldsymbol{\mu}_{\overline{\boldsymbol{r}}}\right)\right) \\
& +\sum_{\boldsymbol{r} \in \mathcal{R}^{\prime \prime}}(-1)^{|\boldsymbol{r}|+\frac{n(n+1)}{2}+1} \operatorname{det}\left(S\left(\mathcal{E}_{n, m, k}(\tau) ; \boldsymbol{r}, \boldsymbol{c}\right)\right) \operatorname{det}\left(\bar{S}\left(\mathcal{E}_{n, m, k}(\tau) ; \boldsymbol{r}, \boldsymbol{c}\right)\right) \\
= & \sum_{\boldsymbol{r} \in \mathcal{R}^{\prime}}(-1)^{|\boldsymbol{r}|+\frac{n(n+1)}{2}} \tau^{2\left|\boldsymbol{\mu}_{\boldsymbol{r}}\right|} \operatorname{GVD}\left(\boldsymbol{x} ; \boldsymbol{\mu}_{\boldsymbol{r}}\right) D_{m, k}\left(\tau ; 3,4, \boldsymbol{\mu}_{\overline{\boldsymbol{r}}}\right) \\
& +\sum_{\boldsymbol{r} \in \mathcal{R}^{\prime \prime}}(-1)^{|\boldsymbol{r}|+\frac{n(n+1)}{2}+1} \operatorname{det}\left(S\left(\mathcal{E}_{n, m, k}(\tau) ; \boldsymbol{r}, \boldsymbol{c}\right)\right) \operatorname{det}\left(\bar{S}\left(\mathcal{E}_{n, m, k}(\tau) ; \boldsymbol{r}, \boldsymbol{c}\right)\right)
\end{aligned}
$$

By Lemma 16 we have:

$$
D_{m, k}\left(\tau ; 3,4, \boldsymbol{\mu}_{\overline{\boldsymbol{r}}}\right)=C_{\boldsymbol{r}} \tau^{1 \cdot\left|\boldsymbol{\mu}_{\bar{u}}\right|+0 \cdot\left|\boldsymbol{\mu}_{\bar{v}}\right|}+\Theta\left(\tau^{1 \cdot\left|\boldsymbol{\mu}_{\bar{u}}\right|+0 \cdot\left|\boldsymbol{\mu}_{\bar{v}}\right|+1}\right)=C_{\boldsymbol{r}} \tau^{\left|\boldsymbol{\mu}_{\bar{u}}\right|}+\Theta\left(\tau^{\left|\boldsymbol{\mu}_{\bar{u}}\right|+1}\right),
$$

where $\overline{\boldsymbol{u}}=\left(2,5, \bar{r}_{5}, \ldots, \bar{r}_{m+2}\right), \overline{\boldsymbol{v}}=\left(3,6, \bar{r}_{m+3}, \ldots, \bar{r}_{m+k}\right)$ and $C_{\boldsymbol{r}}>0$. Hence, for any $\boldsymbol{r} \in \mathcal{R}^{\prime}$, the term in the expansion of $E_{n, m, k}(\tau)$ that corresponds to $\boldsymbol{r}$ becomes:

$$
(-1)^{|\boldsymbol{r}|+\frac{n(n+1)}{2}} C_{\boldsymbol{r}} \tau^{2\left|\boldsymbol{\mu}_{\boldsymbol{r}}\right|+\left|\boldsymbol{\mu}_{\bar{u}}\right|} \operatorname{GVD}\left(\boldsymbol{x} ; \boldsymbol{\mu}_{\boldsymbol{r}}\right)+\Theta\left(\tau^{2\left|\boldsymbol{\mu}_{\boldsymbol{r}}\right|+\left|\boldsymbol{\mu}_{\bar{u}}\right|+1}\right) .
$$

From this expression we deduce that the minimum exponent of $\tau$ for any specific $r \in \mathcal{R}^{\prime}$ is:

$$
2\left|\boldsymbol{\mu}_{\boldsymbol{r}}\right|+\left|\boldsymbol{\mu}_{\overline{\boldsymbol{u}}}\right|<2\left|\boldsymbol{\mu}_{\boldsymbol{r}}\right|+\left|\boldsymbol{\mu}_{\overline{\boldsymbol{r}}}\right|<2\left|\boldsymbol{\mu}_{\boldsymbol{r}}\right|+2\left|\boldsymbol{\mu}_{\bar{r}}\right|=2|\boldsymbol{\mu}| \leq M
$$


where we used the fact that:

$$
\left|\boldsymbol{\mu}_{\boldsymbol{r}}\right|+\left|\boldsymbol{\mu}_{\overline{\boldsymbol{r}}}\right|=\sum_{i=1}^{n} \mu_{r_{i}}+\sum_{i=1}^{m+k} \mu_{\bar{r}_{i}}=\sum_{i \in \boldsymbol{r}} \mu_{i}+\sum_{i \in \overline{\boldsymbol{r}}} \mu_{i}=\sum_{i=1}^{\ell} \mu_{i}=|\boldsymbol{\mu}| .
$$

On the other hand, the terms in (84) that correspond to the row vectors $\boldsymbol{r} \in \mathcal{R}^{\prime \prime}$ cannot be the terms of minimal order of $\tau$, since for these terms the exponent of $\tau$ is greater than $M$. We can thus restrict our attention to the terms for which $\boldsymbol{r} \in \mathcal{R}^{\prime}$, and rewrite (84) as:

$$
E_{n, m, k}(\tau)=\sum_{\boldsymbol{r} \in \mathcal{R}^{\prime}}\left((-1)^{|\boldsymbol{r}|+\frac{n(n+1)}{2}} C_{\boldsymbol{r}} \tau^{2\left|\boldsymbol{\mu}_{\boldsymbol{r}}\right|+\left|\boldsymbol{\mu}_{\bar{u}}\right|} \operatorname{GVD}\left(\boldsymbol{x} ; \boldsymbol{\mu}_{\boldsymbol{r}}\right)+\Theta\left(\tau^{2\left|\boldsymbol{\mu}_{\boldsymbol{r}}\right|+\left|\boldsymbol{\mu}_{\bar{u}}\right|+1}\right)\right)+\Omega\left(\tau^{M+1}\right)
$$

From the expression above, we infer that the term of $E_{n, m, k}(\tau)$ for which the exponent of $\tau$ is minimal is the term for which the quantity $2\left|\boldsymbol{\mu}_{\boldsymbol{r}}\right|+\left|\boldsymbol{\mu}_{\bar{u}}\right|$ is minimized. However, we have that:

$$
2\left|\boldsymbol{\mu}_{\boldsymbol{r}}\right|+\left|\boldsymbol{\mu}_{\overline{\boldsymbol{u}}}\right|=2\left|\boldsymbol{\mu}_{\boldsymbol{r}}\right|+\left|\boldsymbol{\mu}_{\overline{\boldsymbol{u}}}\right|+\left|\boldsymbol{\mu}_{\overline{\boldsymbol{v}}}\right|-\left|\boldsymbol{\mu}_{\overline{\boldsymbol{v}}}\right|=\left|\boldsymbol{\mu}_{\boldsymbol{r}}\right|+\left|\boldsymbol{\mu}_{\boldsymbol{r}}\right|+\left|\boldsymbol{\mu}_{\overline{\boldsymbol{r}}}\right|-\left|\boldsymbol{\mu}_{\overline{\boldsymbol{v}}}\right|=\left|\boldsymbol{\mu}_{\boldsymbol{r}}\right|+|\boldsymbol{\mu}|-\left|\boldsymbol{\mu}_{\overline{\boldsymbol{v}}}\right| .
$$

So, minimizing $2\left|\boldsymbol{\mu}_{\boldsymbol{r}}\right|+\left|\boldsymbol{\mu}_{\overline{\boldsymbol{u}}}\right|$ amounts to determining the vectors $\boldsymbol{r}$ and $\overline{\boldsymbol{v}}$ for which the difference $\left|\boldsymbol{\mu}_{\boldsymbol{r}}\right|-\left|\boldsymbol{\mu}_{\overline{\boldsymbol{v}}}\right|$ becomes minimal. Let $\boldsymbol{\rho}=(1,4,7,8, \ldots, n+4), \overline{\boldsymbol{\rho}}^{\prime}=(2,5, n+5, n+$ $6, \ldots, n+m+2)$ and $\overline{\boldsymbol{\rho}}^{\prime \prime}=(3,6, n+m+3, n+m+4, \ldots, \ell)$. It is trivial to verify that

- $\left|\boldsymbol{\mu}_{\boldsymbol{r}}\right|>\left|\boldsymbol{\mu}_{\boldsymbol{\rho}}\right|$, for all $\boldsymbol{r} \neq \boldsymbol{\rho}$, and

- $\left|\boldsymbol{\mu}_{\bar{v}}\right|<\left|\boldsymbol{\mu}_{\bar{\rho}^{\prime \prime}}\right|$, for all $\overline{\boldsymbol{v}} \neq \overline{\boldsymbol{\rho}}^{\prime \prime}$.

From this observation we deduce that the unique minimal value for $2\left|\boldsymbol{\mu}_{\boldsymbol{r}}\right|+\left|\boldsymbol{\mu}_{\overline{\boldsymbol{u}}}\right|$ is attained when $\boldsymbol{r}, \overline{\boldsymbol{u}}$ and $\overline{\boldsymbol{v}}$ are equal to $\boldsymbol{\rho}, \overline{\boldsymbol{\rho}}^{\prime}$ and $\overline{\boldsymbol{\rho}}^{\prime \prime}$, respectively. Moreover,

$$
\begin{aligned}
|\boldsymbol{\rho}|+\frac{n(n+1)}{2} & =\sum_{i=1}^{n+4} i-(2+3+5+6)+\frac{n(n+1)}{2}=\frac{(n+4)(n+5)}{2}-16+\frac{n(n+1)}{2} \\
& =\frac{\left(n^{2}+9 n+20\right)+\left(n^{2}+n\right)}{2}-16=\left(n^{2}+5 n+10\right)-16=(n-1)(n+6) .
\end{aligned}
$$

Since $(n-1)(n+6)$ is even for any $n$, the term in the expansion of $E_{n, m, k}(\tau)$ corresponding to the minimum exponent for $\tau$ becomes $C_{\boldsymbol{\rho}} \tau^{2\left|\boldsymbol{\mu}_{\boldsymbol{\rho}}\right|+\left|\boldsymbol{\mu}_{\overline{\boldsymbol{\rho}}^{\prime}}\right|} \operatorname{GVD}\left(\boldsymbol{x} ; \boldsymbol{\mu}_{\boldsymbol{\rho}}\right)$. The claim in the statement of the lemma immediately follows from the positivity of $C_{\boldsymbol{\rho}}$ and $\operatorname{GVD}\left(\boldsymbol{x} ; \boldsymbol{\mu}_{\boldsymbol{\rho}}\right)$, and by observing that $2\left|\boldsymbol{\mu}_{\boldsymbol{\rho}}\right|+\left|\boldsymbol{\mu}_{\overline{\boldsymbol{\rho}}^{\prime}}\right|$ equals $\xi$. 